\title{
Platelet aggregation in whole blood
}

Citation for published version (APA):

Burgess-Wilson, M. E. (1996). Platelet aggregation in whole blood. [Doctoral Thesis, Maastricht University]. Datawyse / Universitaire Pers Maastricht. https://doi.org/10.26481/dis.19961101mb

Document status and date:

Published: 01/01/1996

DOI:

10.26481/dis. $19961101 \mathrm{mb}$

Document Version:

Publisher's PDF, also known as Version of record

\section{Please check the document version of this publication:}

- A submitted manuscript is the version of the article upon submission and before peer-review. There can be important differences between the submitted version and the official published version of record.

People interested in the research are advised to contact the author for the final version of the publication, or visit the DOI to the publisher's website.

- The final author version and the galley proof are versions of the publication after peer review.

- The final published version features the final layout of the paper including the volume, issue and page numbers.

Link to publication

\footnotetext{
General rights rights.

- You may freely distribute the URL identifying the publication in the public portal. please follow below link for the End User Agreement:

www.umlib.nl/taverne-license

Take down policy

If you believe that this document breaches copyright please contact us at:

repository@maastrichtuniversity.nl

providing details and we will investigate your claim.
}

Copyright and moral rights for the publications made accessible in the public portal are retained by the authors and/or other copyright owners and it is a condition of accessing publications that users recognise and abide by the legal requirements associated with these

- Users may download and print one copy of any publication from the public portal for the purpose of private study or research.

- You may not further distribute the material or use it for any profit-making activity or commercial gain

If the publication is distributed under the terms of Article $25 \mathrm{fa}$ of the Dutch Copyright Act, indicated by the "Taverne" license above, 


\title{
Platelet aggregation in whole blood
}

\author{
PROEFSCHRIFT \\ ter verkrijging van de graad van doctor \\ aan de Universiteit Maastricht \\ op gezag van de Rector Magnificus, Prof. Mr. M.J. Cohen, \\ volgens het besluit van het College van Dekanen, \\ in het openbaar te verdedigen op \\ vrijdag I november 1996 om 14.00 uur \\ door
}

Michael Edward Burgess-Wilson

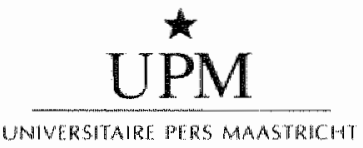




\section{Promotores :}

Prof. Dr. H.C. Hemker

Prof. Dr. S. Heptinstall (University of Nottingham, England)

\section{Beoordelingscommissie :}

Prof. Dr. H.F.P. Hillen (voorzitter)

Prof. Dr. M.P. van Dieijen-Visser

Prof. Dr. G. Hornstra

Prof. Dr. P.W. de Leeuw

Prof. Dr. H.A.M. Neumann

Prof. Dr. G.W.J.M. Tangelder

Mike Burgess-Wilsson. Milpitas Calfornia 1996

Datawyse / Universiaire Pers Maastricht

ISBN 9052782202 


\section{CONTENTS}

\section{Chapter}

Page

Abbreviations

1 Introduction

2 Platelet aggregation in whole blood determined using the Ulitra-Flo 100 platelet counter

3 Spontaneous platelet aggregation in heparinised blood during pregnancy

4 Heparin-induced whole blood platelet aggregation

5 Spontaneous platelet aggregation in whole blood from normal individuals

6 Platelet aggregation in whole blood from patients with Glanzmann thrombasthenia

7 Diamide induces reversible aggregation of human blood platelets

8 The lack of effect of fish ofl supplementation on whole blood platelet aggregation despite changes in platelet fatty acid composition.

9 Studies on platelets before and after stroke in a patient with essential thrombocythaemia

10 The study of whole blood aggregation in a group of patients after suspected myocardial infarction

11 General discussion and conclusions

Summary

Samenvatting

Curriculum vitae 


\section{Abbreviations}

5HT 5-hydroxytryptamine (Serotonin)

$\mathrm{AA}$

Arachidonic acid

ADP Adenosine diphosphate

APTT

Activated partial thromboplastin time

BSS

Bemard-Soulier syndrome

$\mathrm{CB}$

Citrate anticoagulated blood

DVT

Deep vein thrombosis

$\mathrm{EB}$

EDTA anticoagulated blood

EDTA

Ethylenediaminetetra-acetic acid

EPA

Eicosapentaenoic acid

$\mathrm{g}$ acceleration constant

GP

Glycoprotein

GT

Glanzmann thrombasthenia

$\mathrm{HB}$

Heparin anticoagulated blood

$\mathrm{HCl}$ Haematocrit (PCV)

HIA Heparin-induced platelet aggregation

HIT Heparin-induced thrombocytopenia

HPLC High performance liquid chromatography

PAA Platelet aggregometry assay

PAF Platelet activating factor

PBC Platelet count

PCV Packed cell volume (Hct)

PD Potential difference

$\mathrm{PGI}_{2} \quad$ Prostaglandin $\mathrm{I}_{2}$ (Prostacyclin)

PRP Platelet-rich plasma

PPP Platelet-poor plasma

RGDS Arg-Gly-Asp-Ser (a GPIlb/llIa receptor antagonist)

RBC Red blood cells

rpm

SD

Revolutions per minute

SEM

Standard deviation

SIN-1

Standard error of the mean

SPA

SRA

A guanylate cyclase stimulant

$\mathrm{TxB}_{2}$

Spontaneous platelet aggregation

${ }^{14} \mathrm{C}$-serotonin release assay

UF 100

VWF

Thromboxane $\mathrm{B}_{2}$

WB

Ultra-Flo 100 whole blood platelet counter

von Willebrand factor

WBA Whole blood platelet aggregation

WBC White blood cells 


\section{CHAPTER 1}

\section{Introduction}

The measurement of platelet aggregation is one of the most frequently used procedures for evaluating platelet behaviour, and is especially useful in the investigation and diagnosis of platelet disorders (Ludlam 1994). In its most basic form, the test is quite simple; platelets are separated from red blood cells, an aggregating agent is added, the mixture gently agitated and the platelets then clump together forming large visible platelet aggregates. A more quantitative method of measuring platelet aggregation was first described by Jürgens and Braunsteiner in 1950, and was later developed by O'Brien (1962) and Born (1962). This technique, called optical aggregometry, involves measuring the optical changes with a spectrophotometer, rather than using the eye.

Although a large variety of other methods have been developed to study platelet behaviour, optical aggregometry remains the most commonly used method. However, optical aggregometry has several shortcomings; some that are technical, e.g. the need to separate platelets from red blood cells; and some that relate to the methods clinical utility, e.g. the apparent insensitivity of the method to the platelet changes that occur in thrombotic states. When the Ultra-Flo 100 whole blood platelet counter (UF100) became available, it was recognised that this new instrument could be used to measure platelet aggregation in whole blood. Studies were conducted to establish a technique for the measurement of whole blood platelet aggregation (WBA) to avoid the limitations of optical aggregometry. This thesis reports the studies in which I was involved and includes: an initial evaluation which investigated the technical factors involved in the measurement of WBA; further studies which investigated platelets where a change in their behaviour was anticipated; and studies in which the WBA technique was compared with the optical aggregometry technique.

\section{Platelets}

Platelets have been intensively researched, and it is recognised that they are an essential part of the haemostatic mechanism. Individuals with functionally defective platelets may suffer from haemorrhagic or thromboembolic disease. The primary role of platelets appears to be the construction of a 'haemostatic plug' 
which is a complex structure that covers damaged vascular endothelium and blocks lesions within the vessel lining.

Platelet behaviour is rather complicated and involves what appear to be several distinct processes. The first is platelet activation, where changes in platelet membrane receptors lead to a complex biochemical response within the cell (Kroll \& Schafer 1989). Activation may then lead to the other processes: platelet adhesion, where platelets stick or adhere to damaged endothelium, forming a monolayer covering the damaged surface (De Groot \& Sixma 1990); platelet aggregation, where platelets stick to platelets and build a stable "plug" or covering that arrests the haemorrhage; the release reaction, where platelets release their granule contents (Niewiarowski 1994); coagulant activity, where platelets provide a phospholipid surface on which coagulation reactions can occur (Bevers et al 1983); and clot retraction, where platelet metabolic processes bring about the contraction of an actinomyosin-like protein. This causes the platelets to contract and pull together the strands of adherent fibrin, the clot therefore shrinks extruding entrapped liquid (Ratnoff 1994). These processes may be interrelated, for example, ADP released from platelet granules can amplify platelet aggregation, and thrombin formed via the coagulation cascade can activate platelets. It should be noted that when a process is studied in isolation these relationships may be altered and the observed platelet behaviour may be strongly influenced by the test system used.

\section{Platelet aggregation}

Platelet aggregation is the physical platelet-platelet connection which may last for a fraction of a second or be completely irreversible. This process requires calcium ions, fibrinogen, platelet membrane glycoproteins and metabolically active cells. The process is associated with the release of granule contents and changes in the composition of the surface membrane (e.g. the appearance of P-selectin). Aggregation should not be confused with agglutination. Agglutination, which can resemble aggregation, can occur in solutions of metabolically inactive cells, unlike aggregation. In vivo platelet aggregation is one of the main processes that contribute to the formation of 'haemostatic plugs' or thrombi. Platelet aggregates that form within in vitro systems are not comparable with the thrombi that form in vivo. The in vivo thrombus is a complex structure which involves endothelial cells, platelets and the components of the coagulation and fibrinolytic systems. However, the observation of in vitro platelet aggregation is a visible, and hence detectable, measurement of platelet behaviour. This can be compared with the in vitro formation of the fibrin clot, which is a visible manifestation of blood coagulation via the clotting cascade. The measurement of platelet aggregation may therefore be considered as a more general test of platelet function, somewhat 
like the activated partial thromboplastin time (APTT), which is a general test of blood coagulation. Both the APTT and platelet aggregation require several of the components of the systems they measure to be available and therefore abnormal results do not necessarily identify a specific clinical condition. The in vitro observation of platelet aggregation is, therefore, a measurable phenomenon which may reflect some aspect of the thrombus forming mechanism, which is the primary role of platelets. A reduction in the aggregability measured in vitro often relates to a tendency to bruise and bleed abnormally. Conversely, when in vitro platelet aggregability is increased there is often a tendency to form inappropriate thrombi in vivo which may cause or contribute to the various clinical events known as stroke, myocardial infarction, deep vein thrombosis, ischaemic heart disease, etc. the type of clinical event depending upon where the thrombi are formed and which organs they affect. Trip at al (1990) have demonstrated an association between platelet aggregation in witro and both the mortality and frequency of cardiac events in survivors of myocardial infarction.

Experimental evidence suggests that several possible in vivo mechanisms can lead to platelet activation. These mechanisms include synthesis of prostaglandins and thromboxanes, exposure of the blood to the subendothelium (collagen), thrombin formation and $\mathrm{ADP}$ release. Once activated, platelets may then aggregate but the extent of this aggregation depends on the balance of substances present that are pro-aggregatory (platelet agonists) and anti-aggregatory (inhibitors). In vitro the different aspects of the aggregation process may be investigated using different combinations of these agonists and inhibitors. When the objective is to design a test system to imitate the in vivo situation, then physiological agents are commonly used. These substances form a very heterogeneous group and originate from several sources: plasma derived components, such as thrombin, von Willebrand factor and plasmin; catecholamines; vascular products, such as prostacyclin, endothelium-derived relaxing factor, von Willebrand factor and collagen; platelet products, such as $\mathrm{ADP}$, thromboxane $\mathrm{A}_{2}$, prostaglandin $\mathrm{D}_{2}$; and from other sources, such as platelet activating factor from white blood cells. Other aspects of platelet behaviour may be investigated using non-physiological materials, such as ristocetin, aspirin, dipyridamole, diamide and ionophores. These substances do not always use in vivo mechanisms, but switch systems on or off by specific reactions; for example, aspirin turns off prostaglandin synthesis by inhibiting the enzyme cyclooxygenase. These simple non-physiological test systems provide a measurable response, and this may correlate with in vivo platelet activity.

In the clinical laboratory, platelet aggregation studies have several uses. The main use is the routine investigation of unexplained bleeding disorders, although platelet aggregation measurement may be used to assess the viability of platelets 
in concentrated solutions of platelets which are used as therapeutic preparations to treat thrombocytopenia. Optical aggregometry can detect reduced platelet aggregation in several well defined primary disease states, e.g. Glanzmann thrombasthenia (GT), Bernard-Soulier syndrome (BSS) and hereditary storage pool disease. Optical aggregometry can also detect abnormal aggregation in several other disease states where the aggregation defect is secondary to the disease (Bick 1992, Coller 1994, Warkentin \& Kelton 1994). The two most extensively studied disorders, GT and BSS, have clearly defined deficiencies of platelet membrane glycoproteins (Coller 1994). In GT this causes a marked reduction in, or the absence of platelet aggregation when measured using optical aggregometry; agglutination in response to ristocetin is normal. In BSS, platelet aggregation induced by ADP and other agonists is normal, but there is reduced agglutination in response to ristocetin. The platelets are morphologically abnormal and reduced in number, which often makes it extremely difficult to measure the reduced aggregation optically. Whilst being able to detect some bleeding disorders optical aggregometry unfortunately cannot clearly identify individuals who have, or may be at risk of developing thromboembolic disease. These individuals are said to be in a prethrombotic state (Chen and $\mathrm{Wu} 1980$ ). A prethrombotic state appears to exist in many forms; general conditions, such as postoperatively, or in immobilised, elderly or obese individuals; more broadly defined conditions, such as congestive cardiac failure, hyperlipidaemia, hyperviscosity or malignancy; specific disease states, such as diabetes mellitus, homocystinuria, lupus anticoagulant, myeloproliferative disorders, nephrotic syndrome, paroxysmal nocturnal haemoglobinuria or thrombotic thrombocytopenic purpura. Finally a prethrombotic state may be due to a habit or medication, such as oral contraceptive treatment, smoking or, paradoxically, heparin therapy. Not all the individuals in these groups develop thrombotic complications and a method of identifying those at risk is needed. A technique may be used to test individuals after a thrombotic event, but it is often difficult to decide whether the platelet changes are associated with the event, or due to it. If a technique is found to be able to measure changes in platelet behaviour, it can then be used in randomised prospective trials which have long term follow up. These studies, although difficult to organise and complete, offer the best chance of identifying a technique as a prethrombotic marker.

Platelet aggregation studies have been used to evalluate the action of many antiplatelet drugs. Several reports (Trip et al 1990, Akopov 1992, APT Collaboration 1988 and 1994) have shown that treatment with anti-platelet drugs reduces the incidence of thromboembolic disease. Whilst the formation of atheromatous plaque and its rupture are generally accepted as a primary mechanism that causes thrombosis (Born 1979), the involvement of platelets in thrombus formation makes treatment with anti-platelet agents very attractive, therefore making it 
useful to be able to measure any changes in the platelet activity. Evidence suggests that the role of platelets in cardiovascular disease involves platelet aggregation as one of the primary events leading to arterial thrombosis (Akopov 1992), and there are indications that platelet parameters, such as aggregation and platelet volume, can predict ischaemic heart disease (Thaulow et al 1991, Martin et al 1991). The anti-platelet action of any substance is usually defined by the effect within an in vitro system, or on a defined population during a clinical study. Unfortunately many of the clinical trials of anti-platelet agents show a conflicting picture. This has been due to the wide variety of different methods used to measure platelet function, and the testing of population groups that were too small (The APT Collaboration 1988). The detection of a drug-induced in vitro effect is dependent on the method used and the measurement parameter selected, e.g. shape change, adhesion, aggregation, granule content release, etc. It has been suggested that optical aggregometry does not identify some of the drug-induced in vivo effects on platelets (Chen \& Wu 1980). Optical aggregometry was found by others to be less sensitive to the anti-aggregatory effect of prostacyclin when compared with aggregation measured by a particle counting technique (Splawinski et al 1984). Further support for the use of alternative methods to optical aggregometry came from two large clinical studies; one of 308 men in the Caerphilly Collaborative Heart Disease Study (Elwood et al 1990), and another by Abbate et al (1989) which showed that platelet aggregation measured in whole blood was strongly associated with the prevalence of ischemic heart disease.

\section{The measurement of platelet aggregation in whole blood}

In the past 30 years optical aggregometry has produced a much clearer understanding of platelet behaviour, but as previously mentioned, the technique has several limitations. The main ones are associated with the fact that the method relies on changes in light transmission of platelet-rich plasma (PRP) to quantify aggregation. The platelets have to be separated from the red cells by centrifugation which is time consuming, labour intensive and requires a relatively large blood sample. The results are not easily quantified and tend to be subjective. These considerations deter most clinical laboratories from using the technique to screen all the patients with suspected bleeding or clotting disorders. Centrifugation selects a sub-population of platelets from the blood sample because platelets are heterogeneous in size, density and metabolic activity (Karpatkin \& Charmatz 1969, Thompson et al 1982). The sub-population lost in the red cell layer is thought to lack many of the larger and denser cells. The cells present in the PRP may have undergone some degree of activation during centrifugation (Salzman et al 1970). Optical aggregation is insensitive to the formation of small platelet aggregates, because the changes in light transmission only occur when relatively large platelet aggregates form (Thompson et al 1986). 
Opuical agregometry of platele ragregation, but it cannot detect mild to moderately hyper-aggregable platelets. Meporis suggest that it is difficult to identify patients with increased thrombotic tisk using this method, e.g. patients with diabetes, ischemic heart disease, wratin and myeloprolferative disorders (Chen \& Wu 1980, Abbate et al 1989, Bertolino et 1993). This is unfortunate because there are far more patients at risk of developing thrombosis than there are of developing a haemonhage.

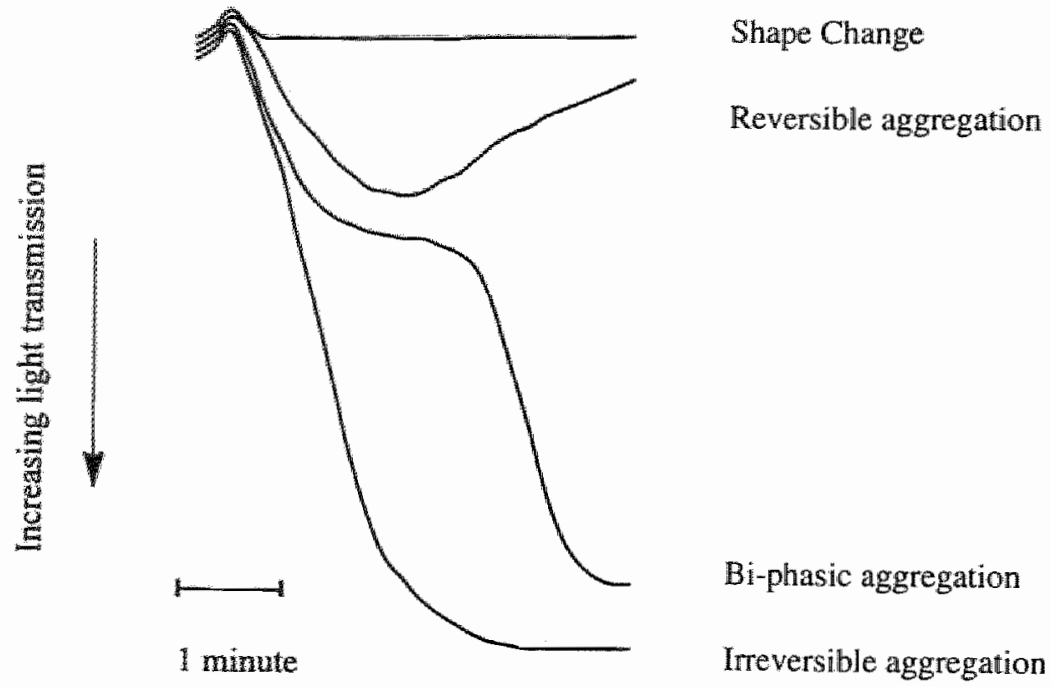

\section{Figure 1}

Fig. 1 Shows typical traces that can be obtained using stirred PRP obtained from a normal individual and an optical aggregometer with ADP as the platelet agonist.

The platelet aggregation observed with optical systems has been classified into 'shape change', 'primary', 'secondary' and 'biphasic' aggregation (Figure 1). Platelet 'shape change' is identified as a decrease in the light transmission usually observed prior to aggregation. Normally discoid platelets change to a more spherical shape, decreasing light transmission. Although platelets do undergo this change in shape, experiments using particle counting and microscopic examination suggest that small aggregates are formed during this period of decreased light transmission. Primary aggregation is a reversible increase in light 
transmission, where platelets aggregate and then disaggregate. The proaggregatory stimulus is strong enough to cause aggregation but not the release of granule contents. As the stimulus fades the platelets disaggregate. Secondary aggregation is a more intense and sustained increase in light transmission, where platelets aggregate irreversibly, releasing the contents of their granules. Biphasic aggregation is occasionally seen where the pro-aggregatory stimulus was just sufficient to initiate granule content release. There is a short lag phase before the additional stimulation provided by the granule contents causes secondary aggregation. The aggregation pattern seen depends on the number of platelets in the PRP sample used, which in turn depends on the separation technique, and on whether the platelet count is further adjusted by adding platelet-poor plasma. These manipulations can create quite different test results (Hendra et al 1988) and there appears to be a critical number of platelets needed in the test system for secondary aggregation to occur. Sometimes the number of platelets in PRP is too low to allow secondary aggregation (Ludlam 1994). Riess et al (1986) showed that the extent of aggregation in PRP observed depends on the time elapsed since its preparation, and that the preparation of the PRP may weakly stimulate the platelets, causing them to become temporarily refractory to certain platelet agonists. All of these observations regarding the use of PRP in optical systems has led investigators to look for other ways to measure platelet aggregation, and one alternative is to use whole blood rather than PRP.

The use of whole blood gives several advantages. In normal citrated whole blood samples the platelet count is closer to that of circulating blood. The whole platelet population of the blood sample is present rather than a selected sub-population, and the platelets are in a more natural environment where interactions with other cellular elements may take place. Red blood cells and white blood cells have been shown to have important positive and negative effects on platelet aggregation (Akopov 1992). Whole blood offers the possibility of measuring aggregation in patients with thrombocytopenia where optical aggregometry would be impossible, e.g. Bernard-Soulier syndrome, and in cases of hyperlipoproteinaemia, where plasma lipids make the sample too opaque for optical aggregometry (Riess et al 1986). Whole blood platelet aggregation (WBA) techniques use smaller blood samples, which is a major advantage where repeated or extensive testing is required, or where the maximum sample volume available is very low, as in paediatric samples. Comparative studies of optical aggregometry and WBA suggest that increased platelet aggregability can be detected more easily in the WBA system (Riess et al 1986, Abbate et al 1989, Akopov 1992). Other investigators use whole blood because the effect of certain drugs, e.g. dipyridamole, is on red blood cells rather than platelets (Violi et al 1991). 
Anticoagulated whole blood samples also have limitations, for example, they cannot be used in an optical system because of the high opacity. Blood is always traumatised by the collection process and then has to be anticoagulated; two processes that significantly affect platelet behaviour. Microscopic examination of native blood drawn through the collection needle shows that some platelet aggregation is already occurring. The anticoagulant sodium citrate reduces calcium ion concentration and there is evidence that platelet aggregation is very sensitive to these changes (Heptinstall and Taylor 1979). The anticoagulant heparin can also influence platelet aggregation (Hirsh 1991). Cells in a blood sample removed from the body are also no longer in contact with the endothelial cells with which they interact (Diodati et al 1993). In native blood, compared to citrated or heparinised blood, thrombin generation occurs much sooner and induces aggregation (Kessels et al 1994, Gorog and Kovacs 1995). The synthesis of this agonist and its inhibition may represent and important part of the platelet aggregation system that cannot be investigated using anticoagulated blood.

\section{Platelets and blood rheology}

Platelets are very sensitive to their environment, especially the forces created by the flow of blood through the different vessels within the body. Blood withdrawn from the body is no longer subjected to the flow and shear conditions within the body that influence both platelet activation and thrombus formation in vivo. If conclusions about platelet behaviour or function in vivo are drawn from in vitro systems, the rheological conditions used in the experiments should be carefully considered, as they will certainly influence the platelet behaviour observed. The physical forces experienced by platelets vary considerably in vivo because blood viscosity, flow velocity, shear rate and shear stress are continuously changing in the flowing blood. These forces are in turn dependent on the proportions of cellular and plasma components in the flowing blood, the vessel geometry and the pumping efficiency of the heart. While, the forces involved are variable they are far from negligible and the platelet behaviour in systems where these forces are missing does not necessarily reflect normal platelet behaviour.

For example, in vivo shear rates from 50 to $3000 \mathrm{~s}^{-1}$ can be found in normal blood vessels; from 50 to $330 \mathrm{sec}^{-1}$ in a main vessel and up to $2800 \mathrm{~s}^{-1}$ in capillaries (Slack et al. 1993). In partially occluded vessels shear rates up to $30000 \mathrm{~s}^{-1}$ have been measured, and these high levels no doubt contribute to platelet deposition. The shear rate influences the blood viscosity, at high shear rates (greater than 200 $\mathrm{s}^{-1}$ ) the viscosity is relatively constant at between 3 and 4 centipoise, but at low shear rates viscosity increases because red blood cells cross-link via fibrinogen (roulleaux formation). Increases in the viscosity then reduce the flow rate. 
Variation in extent of shear stress is important because levels of 12 dyne/ $\mathrm{cm}^{2} \mathrm{~cm}$ cause reversible platelet aggregation, levels of $50 \mathrm{dyne} / \mathrm{cm}^{2}$ the release of ADP from platelet granules, and levels of $100 \mathrm{dyne} / \mathrm{cm}^{2}$ platelet lysis (Slack et al 1993). Shear stress is a platelet agonist, and atherosclerotic lesions can cause high and low shear environments, acting like platelet agonists, causing platelet aggregation.

The flow velocity of the blood also varies not only throughout the body but even within a single blood vessel. The interaction of the blood with the vessel walls reduces the velocity (Figure 2) creating lower flow at vessel walls and higher flow at the axis of the vessel (Goldsmith and Turitto 1986). The flow pattern of blood through stenosed vessels may cause platelet aggregation, and hence thrombus deposition, suggesting that haemostatic effects are intrinsically dependent on flow (Slack et al 1993). Blood flow also changes the distribution of the cellular components in relation to each other. Red blood cells and platelets are not uniformly distributed across the vessel lumen; there is a higher concentration of $\mathrm{RBC}$ at the axis with more platelets at the vessel walls. Tangelder et al (1985) using fluorescence microscopy showed that in rabbit arterioles (mean diameter: $27 \mu \mathrm{m})$ platelets were concentrated at the vessel walls, and suggest that the presence of RBC cause this distribution. Slack et al (1993) showed that there can be up to a 5-10 fold concentration in platelets. Using the same technique Woldhuis et al (1992) confirmed the concentration of platelets at the arteriole wall but showed that in venules there is a concentration of platelets in the centre of the vessel. They also found the platelet distribution found in venules in vivo shows no correspondence with that obtained in vitro tube systems. The nature and origin of this effect are obscure but it is thought to be due to the collision of platelets with red blood cells which augments platelet movement towards the vessel wall and from a low to a high shear environment. The distribution pattern in arterioles certainly seems favourable with regard to the platelets main role but it is difficult to explain the apparent exclusion of platelets from venule walls. Woldhuis et al (1992) suggested that the development of a specific distribution may either require a minimum length of vessel which is available in the arterial system but not in the venous system, or that the blood flowing in from side branches in the venous system pushes the cells away from the vessel wall.

The binding of adhesive proteins that are involved in the aggregation process is also dependent on the rheological forces. Low shear stress levels induce binding of fibrinogen to glycoprotein IIb/IIIa complex (Russell-Smith et al 1981), whereas at higher levels of shear von Willebrand factor ( $\mathrm{WWF}$ ) and glycoprotein Ib/IX interact (Jen \& McIntire 1984). Evidence suggests that the $\mathrm{VWF}$ is involved in shear dependant thrombus formation (Weiss et al 1989, Roth 1991. Ruggeri \& Ware 1992). In one particular experiment which involved the measurement of 
platelet deposition in a flow system (Ruggeri 1993), a synthetic WWF inhibitor was added to blood stirred at different shear rates. Without the inhibitor the platelet deposition increased with increasing shear stress, being minimal at 100 $\mathrm{sec}^{-1}$. However the addition of the VWF inhibitor reduced platelet deposition at all shear rates $\left(100-3000 \mathrm{sec}^{-1}\right)$ to a level just below that seen at $100 \mathrm{sec}^{-1}$.
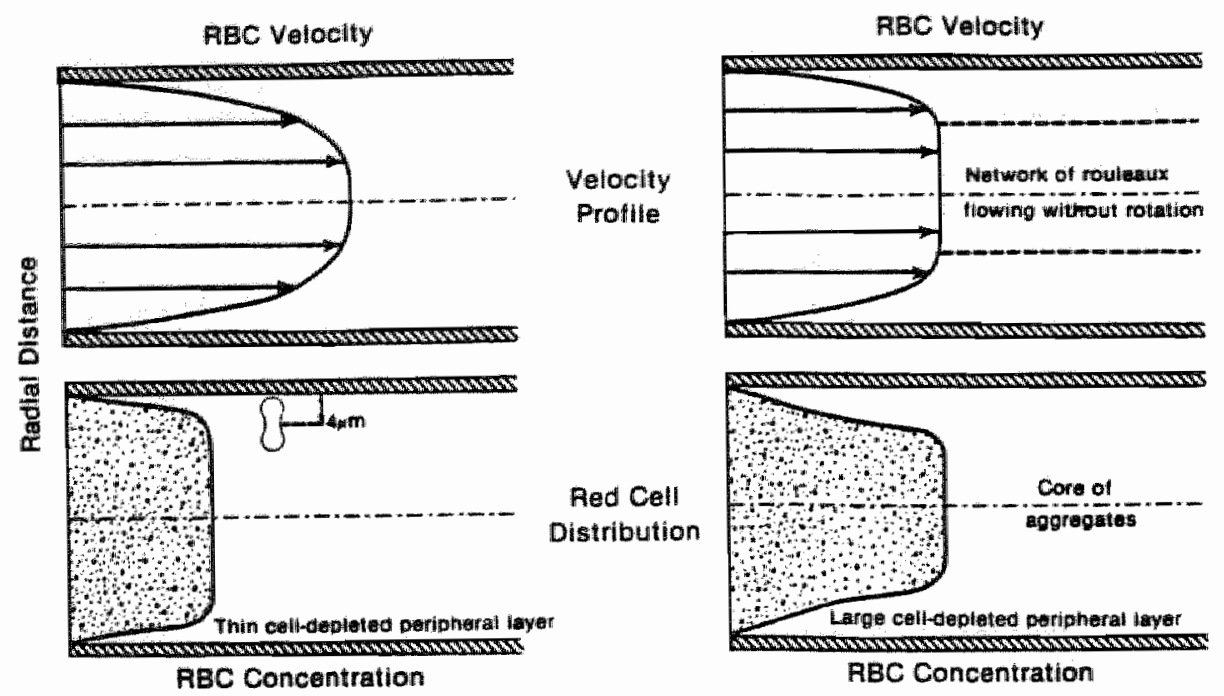

Figure 2

Fig 2.

The velocity profile of flowing blood and the RBC distribution across the vessel (Goldsmith and Turitto 1986).

Although the effects of blood flow are complicated, they show that platelets are very versatile, and are able to adapt to the different environments of the vascular system. The platelets are normally, therefore, activated by the rheological conditions generated by a lesion in the vessel wall. Unfortunately, abnormalities of the vessel wall can mimic or create lesions which then lead to platelet deposition and thrombus formation, and in severe cases to total vessel occlusion.

Aggregometers cannot be used to study platelet function at the shear rates encountered in vivo because they work at low-shear, where the platelet-platelet interactions are primarily fibrinogen-dependent events, although von Willebrand factor can substitute for fibrinogen in experiments using washed platelets (Ikeda et al 1991). Viscometers have the important advantage of being able to produce quantifiable shear rates, subjecting blood samples and platelets to physiological shear forces. These forces can aggregate platelets without the addition of exogenous agonists. This 'shear-induced' platelet aggregation depends on the type of anticoagulant. For example, platelet aggregation occurs at low shear stress 
with low calcium ion concentrations (using citrate as anticoagulant), but this aggregation is absent or markedly reduced at normal calcium ion concentrations (using hirudin as anticoagulant). This suggests that in low shear stress environments, the stable platelet aggregates that form in wiro may not actually form in vivo (Ikeda et al 1991). Viscometers, although useful in the evaluation of shear-induced aggregation, are more complicated in their use than optical aggregometers and consequently are not used as frequently in clinical laboratories. Haemostasis and thrombosis also depend on blood flow to supply "raw" materials, new cells and remove waste products and non-adherent cells. The only systems that can duplicate this are those that use a continuous supply of native blood such as the filtragometer (Hornstra \& ten Hoor 1975) or perfusion chambers (Baumgartner 1973). However, the requirement of native blood severely limits the use of these techniques within the routine clinical environment.

\section{The interaction of platelets with other blood cells}

Although studies of whole blood in stirred systems cannot accurately mimic in vivo flow conditions, they can investigate the influence of other blood cells on platelet behaviour. The effects of red and white blood cells on platelets probably depend on both the blood flow conditions and on the proportion of cells present, which may explain the very different observations and conclusions reached in the study of these interactions.

Red blood cells (RBC) have both physical and chemical effects on platelets. Turitto \& Weiss (1980) have shown that RBC encourage platelets to diffuse towards the vessel wall. Saniabadi et al (1985) have suggested that platelets collide with RBC and in the process become activated and attached to the RBC, the attached platelets becoming a focus for the formation of a larger platelet aggregate. The size and the deformability of RBC, and the RBC mass have been associated with the effect of RBC on platelets (Aarts et al 1983). One of the chemical effects of RBC is the release of the platelet agonist ADP (Gaarder et al 1961, Harrison \& Mitchell 1966, Saniabadi et al 1985). RBC are also a source of haemoglobin, which is a scavenger of the platelet aggregation inhibitor nitric oxide (Diodati et al 1993). The rate of release or "leakage" from the $\mathrm{RBC}$ of these substances, and hence their effect, can be influenced by substances that increase membrane fluidity and permeability (Kalra et al 1994). Support for the theory that ADP released from $\mathrm{RBC}$ can potentiate platelet aggregation comes from experiments where ADP-utilising enzyme systems were shown to reduce shear. induced platelet aggregation (Russell-Smith et al 1981, Saniabadi et al 1984 \& 1987), although other investigators were not able to confirm these reports (Shapiro \& Williams 1970). Additional support for this argument comes from the observation that monoclonal antibodies against the glycoprotein IIb/IIIa complex 
can abolish stirring-induced whole blood platelet aggregation (Falcon et al 1989 , Cook et al 1993). Other evidence that RBC can potentiate platelet aggregation in stirred whole blood comes from experiments that correlate increasing packed cell volume (PCV) with aggregation (Samiabadi et al 1985). Cadroy and Hanson (1990) showed that thrombus formation in primates is dependent on the PCV and in a rabbit blood perfusion system Turitto and Weiss (1980) found that the a greater number of thrombi formed as the PCV was increased. It has been suggested that arachidonic acid released from the RBC membrane may be converted to thromboxane $\mathrm{A}_{2}$ which is a powerful short-lived platelet agonist. RBC may potentiate aggregation by binding prostacyclin, which is a powerful anti-aggregatory agent (Willems et al 1983). Changes in RBC morphology may also influence platelet behaviour. The size of the $\mathrm{RBC}$ has been related to $\mathrm{RBC}$ induced aggregation (Aarts et al 1982), and less deformable RBC are thought to increase platelet diffusion and make them more lysable, hence releasing proaggregatory chemicals (Saniabadi et al 1985). Platelets can also affect RBC by releasing substances that reduce RBC deformability (Palinski et al 1983). Changes in blood viscosity may also influence platelet behaviour. The difference in viscosity between whole blood and PRP may also account for different platelet aggregability as PRP has only a quarter of the viscosity of whole blood. A standard shear force that can cause aggregation in whole blood will exert only a quarter of the shear stress in PRP. Others think that the presence of RBC in a test sample might inhibit very large aggregate formation, and this is supported by the observation that aggregates are smaller in whole blood. Another regulatory role of RBC may be their ability to take up serotonin (Pérez-Requejo et al 1995).

In vivo both platelets and WBC are concentrated somewhat at the vessel wall, which leads to an environment that in theory allows firequent physical contact between these cells. Platelet and their released products can affect WBC, e.g. neutrophil chemotaxis, adhesion, aggregation and granule content release (Bazzoni et al 1991). Akopov (1992) reviewed the effects of WBC on platelets and listed several observations showing a pro-aggregatory effect. The supernatant of leucocytes has been shown to stimulate platelet activity, WBC have been shown to release platelet activating factor (PAF), thromboxane $\mathrm{A}_{2}$, leukotrienes $C_{4}$ and $D_{\sharp}$ and superoxide anions, all of which are pro-aggregatory. Akopov also described experiments that suggested that WBC production of superoxide dismutase can inhibit aggregation. Platelet aggregation in whole blood has been shown to be inversely related to WBC number (Abbate et al 1986, Abbate et al 1989). This relationship may be due to the ability of WBC to produce platelet inhibitors and to release anti-aggregatory substances like prostacyclin $\left(\mathrm{PGI}_{2}\right)$ and inactivate pro-aggregatory substances like ADP (Blackwell et al 1978). Platelet binding sites are formed on WBC by certain platelet agonists, and there appears to be "co-operation' of platelets and WBC in the production of both prostacyclin 
(Wu et al 1987) and other pro-aggregatory substances like PAF and leukotriene $\mathrm{C}_{4}$ (Jungi et al 1986). Mixtures of WBC and platelets can produce more massive thrombi than similar numbers of platelets or WBC tested separately (Akopov et al 1991). In a platelet-neutrophil system, Valles et al (1993) showed that neutrophils can reduce platelet activation and their ability to recruit or activate other platelets. This inhibitory effect increased when P-selectin was inhibited, suggesting that the adhesion of platelet and neutrophils may be prothrombotic. P-selectin has also been shown to enhance leukocyte accumulation within thrombi (Palabrica et al 1992).

Other cellular interactions that might indirectly influence platelet behaviour have been described (Akopov 1992). RBC can augment WBC (as well as platelet) movement towards the vessel wall, the effect is dependant on the PCV; WBC are able to reduce $\mathrm{RBC}$ deformability; endothelial cells can release pro- and antiaggregatory substances, e.g. $\mathrm{PGI}_{2}$, nitric oxide, endothelin-1, thromboxane $\mathrm{A}_{2}$ and endoperoxides.

\section{Methods available for the measurement of whole blood platelet aggregation}

Several different systems have been developed that can measure platelet aggregation in whole blood (Table 1). However many of these systems are only used in research laboratories because they require special apparatus or materials, are expensive and/or require considerable expertise to perform the test and to interpret the results.

\section{Flow Cytometry}

Changes in the structures present on the outer platelet membrane have been associated with platelet activation and aggregation. The technique of flow cytometry can be used to measure phenomena that occur simultaneously with, or as a consequence of aggregation, e.g. microvesicle shedding (Patscheke and Ruff 1995), or granulocyte-platelet and monocyte-platelet aggregate formation (Portele and Verhallen 1995). The technique does not, however, measure platelet aggregation directly.

\section{Perfusion chambers}

Since this technique was first described by Baumgartner (1973) there has been an increasing awareness of the importance of fluid flow on thrombus formation and platelet aggregation (Slack and Turitto 1994). Baumgartners" original technique studies the results of perfusing blood through everted abdominal rabbit aorta vessel segments, which had been denuded of endothelial cells. Rabbit and human arteries were subsequently shown to behave in a similar fashion using this system 
(Tsehopp et al 1979). The technique evaluates platelet and fibrin deposition on the subendothelium and after the perfusion the vascular segment is fixed and histologically processed, so that the cellular components may be analysed morphologically. Thrombus deposition at the different shear rates that exist in wivo can be studied by adjusting the annular width of the chamber. The platelet aggregation seen is strongly influenced by the configuration (parallel-plate, tubular and annular) and contents (blood vessel segments, etc.) of the perfusion chamber. The technique can investigate either anticoagulated blood or nonanticoagulated (native) blood. The latter removes the interference of an anticoagulant but testing requires a larger blood sample. Adjustment of the annular width can only reduce the sample volume to about $60 \mathrm{ml}$, which is still too large for routine or repeated testing. Studies using anticoagulated blood may, however, be made with smaller, more acceptable volumes (e.g. $15-30 \mathrm{ml}$ ). This technique has shown that platelet interaction is favoured by increasing shear rate whilst fibrin deposition is negatively influenced. Adhesion of platelets to the subendothelium is strongly dependent on shear rate, being greater at shear rates above 650 per second.

\section{Table 1 Systems for measuring whole blood platelet aggregation}

\begin{tabular}{|c|c|}
\hline \multirow{2}{*}{ Perfusion chambers } & Parallel plate \\
\cline { 2 - 2 } & Annular \\
\hline Viscometers & Tubular \\
\hline Filter-blocking & Cone and Plate \\
\hline Hole-blocking & Coutte \\
\hline Filtragometry \\
\hline Blood-stiming/mixing & Haemostatometer \\
\hline & Thrombostat (PFA) \\
\hline
\end{tabular}

The role of RBC in platelet aggregation has been supported by several observations using perfusion systems. Platelet deposition using whole blood was up to 60 times greater than that observed when PRP was used. Further support came from experiments where blood was separated into its different cellular fractions, (e.g. RBC, platelets). These fractions were then used to reconstitute whole blood samples to allow the study of the effect of changes in the platelet count and the haematocrit. These experiments showed that platelet deposition was significantly reduced when the haematocrit fell below $30 \%$, and a low 
haematocrit and platelet count were negatively synergistic. When RBC were transfused into an anaemic, thrombocytopenic patient, perfusion studies showed increased platelet adhesion and aggregation, with increased fibrin deposition.

Perfusion studies have been used clinically to test the efficacy of factor VIII-vWF preparations designed to treat patients with von Willebrand Disease, to test the quality and function of stored platelets, both refrigerated and cryopreserved and to assess the platelet function of thrombocytopenic patients before and after platelet transfusion. Perfusion systems have also been used to investigate the actions of potential anti-thrombotic drugs, e.g. specific thrombin inhibitors compared with heparin, and the effect of aspirin with variable shear rates (Barstad et al 1995). Even though perfusion techniques have many useful applications they are usually only found in research-orientated environments because of the technical complexity, expense, tissue sample requirements and large blood samples needed. Nevertheless they have provided important information on fundamental aspects of haemostasis, and it is clear that the perfusion technique will remain a valuable experimental model.

\section{Viscometers.}

Viscometers allow blood to be exposed to precisely controlled and uniform shear stresses and have been used extensively for studies of shear-induced aggregation (Ikeda et al 1988, O'Brien 1990, Ikeda et al 1991, Mazeaud et al 1994). In viscometers the entire fluid phase is subjected to uniform forces. In these systems one solid surface is fixed, whilst the other moves, which creates a velocity gradient. The study of platelet aggregation induced by shear in the absence of agonists has increased the understanding of the potential mechanisms underlying thrombi formation, especially in occluded vessels where shear stress is very high. Like the perfusion technique, viscometers are found most commonly in research environments. Most investigators prefer to use platelet-rich plasma and the technique is seldom used in the diagnosis of patients with hyper- or hypo-reactive platelets.

\section{Filter-blocking techniques.}

Filter blocking techniques involve pumping blood through a filter which resists the passage of the cells. The filter architecture and the shear forces present cause the cells to become lodged within the filter and eventually to block it. The time that the filter takes to block and the changes in pressure either side of the filter can be recorded and several investigators have developed systems that exploit this effect. The Filtragometer method, first described by Hornstra and ten Hoor (1975) uses blood pumped directly from an arm vein, that is with either citrate, heparin or hirudin. The anticoagulated blood then passes through a nickel filter whilst the pressure on each side of the filter is measured. The pressure rises as the 
filter is blocked by both platelets and red blood cells. Aspirin and prostaglandin $E_{1}$ reduce filter blockage in vitro and in wivo. In the original description of the method the authors suggest that circulating aggregates can be detected. Another method called the filter 'bleeding time' has been described by O'Brien (1980). In this method native or anticoagulated blood is forced through a glass fibre filter and the platelet retention within the filter, the time taken to block the filter and number of platelets required, are measured. These methods are good at detecting von Willebrand disease, Glanzmann thrombasthenia and Bernard-Soulier syndrome and are both quick and simple to use. However, their use within clinical laboratories is rarelly reported. This is perhaps because the laboratory must set up and calibrate their own equipment and cannot buy a 'package' off the shelf.

\section{Hole-blocking techniques.}

This principle is quite similar to the filter-blocking technique but in this technique blood is forced through a small hole which becomes blocked. The change in pressure and time taken to occlude the hole are measured. Platelet aggregation and platelet adhesion are both measured by techniques which use filter-blockage or hole-blockage, therefore the results may not correlate directly with methods that measure only platelet aggregation. The Haemostatometer was first described by Görög (1986) and later in a more developed form by Kovacs et al (1989). Native blood is forced through a small bore tube which is then punctured, allowing the blood to escape out of the holes and a plug then forms blocking the holes. Fibrinolytic agents can be added and then the rate of plug dissolution may be measured. The pressure in the system relates to the patency of the hole. Measuring the speed at which the hole can block or un-block is a measurement of the haemostatic ability of the blood which includes its aggregability. This technique is commercially available Xylum CSA Clot Signature Analyser (Sloane and Blake 1991). There is another commercial system that uses this technique, the Thrombostat 4000, developed by Kratzer and Born (1985), and more recently as the Platelet Function Analyser (PFA-100) (Mammen 1996). This system is different, in that it forces blood through a hole punched in a membrane. Like the filter-blocking techniques, this methodology has not enjoyed widespread use, but with the advent of the PFA-100, which represents a major advance in convenience of use, more widespread usage may be expected.

\section{Blood-stiming/mixing techniques.}

There are two main semi-automated techniques that use stirring or mixing; the impedance method and the particle counting method. These two techniques use different measuring principles and the mechanisms by which platelet aggregates form are quite distinct. 
Impedance aggregometry of platelets was first described in 1979 by Flower and Cardinal and is now quite widely used. The technique requires only small blood samples, is quick and simple to use and can be used with either washed platelets, PRP, filtered platelets or whole blood. It can detect platelet aggregation in samples with hyperlipoproteinaemia when it is not possible to do so using optical aggregometry. Impedance aggregometry measures the electrical conductivity (impedance) between two fine platinum wires $1 \mathrm{~mm}$ apart suspended about $1 \mathrm{~cm}$ into a stirred sample of whole blood. The conductivity of normal citrated whole blood takes 2 to 3 minutes to stabilise after the electrodles are in contact with the blood sample. This is due to the platelets adhering to the electrodes, forming a monolayer of platelets. If aggregation is stimulated, platelets join this monolayer by cell-cell aggregation causing a change in the impedance between the electrodes which can be measured and used to quantify platelet aggregation. In the original report no change in impedance was observed that could be attributed to platelet 'shape change'. Most reports compare impedance aggregation with optical aggregation, although a study by Karlberg et al (1993) compared filtragometry with impedance aggregometry and showed similar results. However comparisons of impedance aggregometry with optical aggregometry suggest that the aggregation seen with impedance aggregometry is different to that seen with optical aggregometry (Hendra and Yudkin 1990). Ingerman-Wojenski et al (1983) reported that disaggregation of platelets could not be measured using impedance aggregometry, and observed a delay between the onset of aggregation and the change in impedance. Musumeci et al (1987) reported that RBC can inhibit impedance aggregometry, and noted that the baseline impedance depended upon the PCV of the sample and the stirring conditions. The addition of increasing amounts of plasma was shown to potentiate stirring-induced impedance changes, and this dependence was not seen using PRP. Musumeci also reported that the extent of collagen-induced aggregation increased with decreasing $P C V$, i.e. the $\mathrm{RBC}$ seemed to inhibit platelet aggregation. He further observed that in human whole blood impedance decreased immediately after agonist addition, the extent being more marked as the PCV increased. This observation is sometimes compared to the observation of "shape change" seen in PRP, but this is difficult to accept because in whole blood this phenomenon is PCV-dependant. Musumeci concluded that in clinical conditions where the PCV and concentration of plasma protein changed, there would be changes in the aggregation pattern observed that where not due to changes in platelet aggregability. He suggested, therefore, that when impedance aggregometry was used the PCV of the test sample should be adjusted to between $20-25 \%$ to remove this artefact. The original studies of impedance aggregation used rabbit blood, which has a lower RBC to platelet ratio than human blood, so it was not surprising that no decrease in impedance with the addition of agonist was seen. An observation that suggested that the technique could not detect platelet shape change (Cardinal and Flower 1980). Ingermann- 
Wojenski et al (1983) also reported an intial decrease in impedance following agonist addition which was PCV-dependent and suggested that it was due to the presence of large numbers of RBC around the electrodes. Musumeci suggested that RBC form aggregates which influence the impedance only when the PCV is high. Riess et al (1986) studied impedance changes in PRP and in whole blood which they diluted 1 in 2 with saline simultaneously, and compared the results with the optical aggregation measured in PRP samples. They observed that whole blood platelet aggregation (WBA) was slower than platelet aggregation in PRP, although similar results were obtained in PRP and whole blood when ADP or collagen were used to induce aggregation. They also observed platelet 'shape change' in both PRP and whole blood; these results being at variance with other workers (Ingermann-Wojenski et al 1983), but Riess concluded that the dilution of the whole blood allowed 'shape change' to be observed. He also suggested that shape change of the platelets on the electrodes might change the impedance, and that $\mathrm{RBC}$ might interfere with impedance aggregation. The latter theory was supported by the work of Macchi et al (1984). Riess et al (1986) also found that aggregation in whole blood needed lower concentrations of thrombin, ristocetin and arachidonic acid than PRP, even when the PRP platelet count was adjusted to be comparable with the whole blood sample. Hendra et al (1988) found that lower concentrations of either ADP, adrenaline or collagen were needed to induce aggregation in whole blood than in PRP. It is worth noting that no author seems to have considered the fact that if the agonist cannot freely enter the space occupied by the RBC, so the concentration of agonist in per unit volume of whole blood may be almost double that per unit volume of PRP. It would be interesting to know how the final concentration of agonists used in whole blood and PRP was calculated, as this may explain the differences reported. Reiss further suggested that the platelet counts of the whole blood or PRP studied (whether diluted or not) may influence the experimental results because a higher platelet count would lead to more cell-cell collisions. Impedance aggregometry has also been investigated as to its suitability for detecting platelet hyper-activity. The extent of WBA using this technique was increased when compared with aggregation measured in PRP in both ischaemic heart disease and diabetes (Abbate et al 1989), both conditions where increased platelet reactivity has been previously reported. This suggests that impedance aggregometry may be preferable to optical aggregometry in the study of platelet hyperaggregability.

Particle counting techniques have been used for many years to measure platelet aggregation. Before automated electronic cell counters were available platelets were counted microscopically either in counting chambers or on stained blood films. Electronic particle counters made the technique much quicker and easier. These instruments have been designed to count the number of particles of a selected size, and are based on one of two principles. The most commonly used is 
the 'Coulter principle', where a potential difference is set up across a small orifice through which cells are passed. As the cell momentarily blocks the orifice in its passage, it changes the resistance between the two sides of the orifice, and this change is amplified and recorded. The change in resistance is proportional to the size of the particle, and particles of only a particular size range may be selected by counting resistance changes of a certain magnitude. The second principle uses the measurement of light scatter by particles as they are channelled, single file, down a narrow tube. The extent and nature of the light scatter allows. cell identification and calculation of number of particles with similar properties. Platelets are heterogeneous bodies ranging from 2-25 $\mathrm{fl}$, and particle counters may be calibrated to measure only the particles in this size range. When platelets aggregate, the particle counter will count each aggregate as a single particle. So in an aggregating sample the measured particle count, within the platelet size range, will decrease and this change can be used to quantify platelet aggregation. In theory, any instrument that can count platelets may be used. Cheeseman et al (1984) showed that the ELT 8/ds automated blood cell analyser, which performs routine blood counts, could be used to measure platelet aggregation. The technique described is, however, laborious and could not easily be adjusted to the routine testing of platelet aggregation. McLaren et al (1990) reported a good correlation between platelet aggregation measured using the UF100 with a Coulter counter T-540 whereas Barradas et al (1992) compared the Coulter T-890 whole blood counter with impedance aggregometry. They found that the platelet counting technique was more sensitive, rapid, versatile and simpler to use compared to the impedance technique. These techniques, however, require extensive use of the analysers to perform all the testing, and this is not always possible in clinical laboratories.

\section{The Ultra-Flo 100 whole blood platelet counter (UF100)}

Around 1976 several attempts where being made to improve the sensitivity of platelet aggregation measurement by counting single platelets (Hendra and Yudkin 1990). Both visual and automated techniques were being used to measure aggregation in PRP and whole blood, but the methods developed were usually difficult and time consuming. The UF100 was first available in 1976 and counts platelets using the 'Coulter principle'. The initial evaluation studies verified that single platelets in whole blood were counted accurately (Day et al 1980, Bacus ef al 1980). When first available, the UF100 had an important new feature, 'hydrodynamic focusing' (Figure 3). The diluted whole blood is forced by positive pressure up through the 'sample tube'. The fluid flow is so arranged that the cells are focused through the orifice and then removed rapidly on the other side of the orifice up the 'catcher tube'. Hydrodynamic focusing improves the accuracy of the particle count. Without this feature, when liquid containing cells is forced 
through an orifice some of the cells approach the orifice so closely (but without going through) that the resistance measurement changes enough to register as a particle counted. Simillarly, once through the orifice, cells may circulate back very close to the orifice in eddies, and get re-counted. These erroneous 'counts' produce a falsely elevated particle count, and removal of this artefact improves the accuracy of the platelet count.

The UF100 has another unusual feature, it counts the platelets in relation to the $\mathrm{RBC}$ present, and a minor disadvantage of the UF100 is that the machine requires red cells present in the sample before it can determine a platelet count. This means that aggregation in PRP can only be measured if RBC are added to the diluted sample that is counted by the machine. The advantage of this feature is that the dilution of blood sample in diluent need not be exact, as the ratio of RBC to platelets is used to calculate the platelet count.

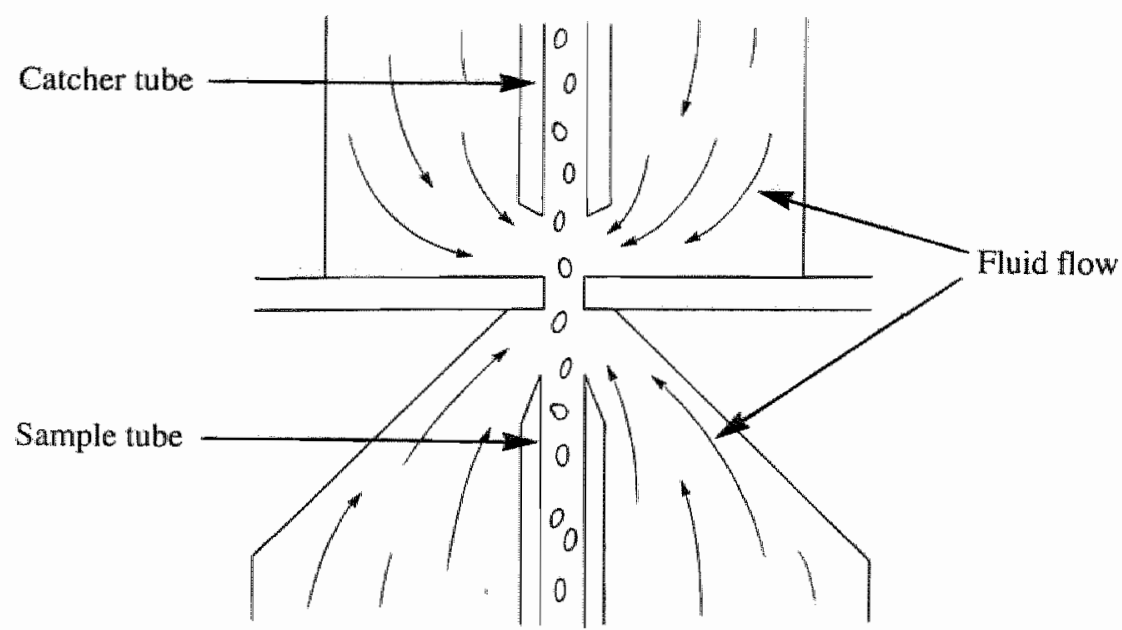

Figure 3

Fig. 3. A schematic diagram of the hydrodynamic focusing method used in the Ultra-Flo 100 Whole Blood Platelet Counter.

The use of the UF100 for platelet aggregation studies was first described by Butchers et al (1980). Between 1980 and 1985 several groups investigated the UF100 including; Turitto et al (1980), Fox et al (1982), Saniabadi et al (1983), Cheeseman et al (1984) and Harrison et al (1984). All these groups obtained basically similar results, although the sensitivity of the method appeared to depend on subtle differences in methodology (Falcon et al 1989). Differences centred mainly on the collection and handling of blood prior to testing, and then 
the removal and counting of samples to measure aggregation. Fox et al (1982) and others showed that the number of single platelets in the preparation gradually fell during storage. To remove this variable their testing was performed at a standardised time after venepuncture. They developed two methods of removing aliquots to measure platelet aggregation. In the first method an aliquot, typically $10 \mu l$, was transferred directly into saline diluent and counted on the machine. The dilution of the whole blood in this fashion prevented further aggregates forming

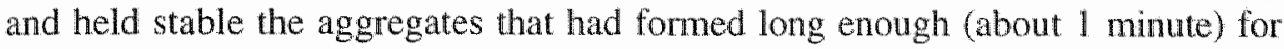
the number single platelets to be accurately counted (Fox et al 1982). When multiple samples were removed faster than the platelets could be counted an alternative technique was used. In this technique the aliquot of whole blood was added to a formaldehyde solution which 'fixed' the platelets (Bevan and Heptinstall 1985). Testing showed that the number of single platelets in these solutions of 'fixed' platelet remained stable for several hours. Other investigators (Butchers et al 1980) preferred to incubate a shaking whole blood sample for 30 minutes at $37^{\circ} \mathrm{C}$. This equilibrated the mixture so that the number of single platelets left in the sample remained constant during further testing.

One aspect that many authors found useful to study was "spontaneous platelet aggregation' which was abbreviated to 'SPA'. This term is, of course, a misnomer because the phenomenon is certainly not spontaneous; however, the more accurate term of "Stirring induced anticoagulated blood platelet aggregation" does not roll off the tongue very easily. The term SPA therefore usually denotes stirring or mixing-induced platelet aggregation. SPA has allso been observed in stirred PRP, but the frequency and extent are much lower than in stirred whole blood (Bertolino et al 1993). SPA is often only measurable for a short period in the life of the PRP, whereas it is continually present in whole blood. The extent of SPA in whole blood depends on the extent of mixing (stirring), the temperature, the type of anticoagulant, the PCV and the amount and type of surface contact present in the system. It was suggested that ADP leakage from RBC contributed to the SPA and enhanced the response to agonists (Lowe et al 1985, Splawinska et al 1990).

Investigators also observed in whole blood reversible platelet aggregation with low platelet agonist concentrations, with irreversible aggregation at high concentrations, and a dose-dependant response to agonists, similar to that seen using PRP. When 5HT inhibitors were used, the expected effects were seen by Bevan and Heptinstall (1985). 


\section{Platelet aggregation techniques reported between 1992 and 1994}

A review of the issues of Thrombosis Research and Thrombosis and Haemostasis published between January 1993 and November 1994 was used to perform a crude survey of the different types of techniques then being employed to measure platelet aggregation. Most of the papers were submitted to the journals between 1992 and the first half of 1994 and Table 2 shows the results of this survey.

There were 133 reports of platelet aggregation studies. The optical technique was reported in $79 \%$ of the reports. Most of these reports entailed the use of PRP $(61 \%)$, with the others using washed platelets $(18 \%)$. In $11 \%$ of all the reports impedance aggregometry was used whereas in $10 \%$ of all the reports particle counting was used. This survey differs slightly from McLaren et al (1990) who reported in 1990 that the particle counting technique was the most common technique used for whole blood platelet aggregation studies. During 1994 there were isolated reports of the use of the Thrombostat 4000 , the perfusion system, the filter 'bleeding time' and gas bubble-induced platelet aggregation. Instruments most commonly cited in reports of platelet aggregation testing were the Chronol.og and Payton series of machines. Many ChronoLog instruments can test whole blood, PRP or washed platelets, but most investigators preferred to use only PRP. Particle-counting techniques (10\%) and the impedance methods (11\%) were cited at a similar incidence, and generally tested whole blood samples. All reported impedance testing used the ChronoLog instruments and although no longer obtainable, the UF 100 was used in about half the reports of particle counting methods using whole blood, Coulter counters being used in nearly all other reports.

Table 2 Platelet aggregation techniques 1992-1994

\begin{tabular}{|c|c|c|c|}
\hline Technique & Material & $\begin{array}{c}\text { Number of } \\
\text { references }\end{array}$ & Percentage (\%) \\
\hline \multirow{2}{*}{ Optical } & PRP & 81 & 61 \\
\cline { 2 - 4 } & Washed platelets & 24 & 18 \\
\hline \multirow{3}{*}{ Impedance } & Whole Blood & 13 & 10 \\
\cline { 2 - 4 } & PRP & 2 & 1 \\
\cline { 2 - 4 } & Washed platelets & 0 & 0 \\
\hline Particle counting & Whole blood & 9 & 7 \\
\cline { 2 - 4 } & PRP & 3 & 2 \\
\hline Total & Washed platelets & 1 & 1 \\
\hline
\end{tabular}




\section{This investigation}

This thesis describes experimental work performed using the Ultra-Flo 100 Whole Blood Platelet Counter to measure platelet aggregation in whole blood. The work consists of the initial evaluation of the machine circa 1982, and the development of a methodology and its application to blood samples from individuals with normal and abnormal haemostasis between 1982 and 1989. This was not hypothesis-based research, it was the investigation of a new, potentially useful technique for the measurement of platelet aggregation in whole blood. 


\section{References}

Aarts PAMM, Sixma JJ, Sakariassen KS, Bolhius PA and Heethaar RM. The infuence of red cell size on platelet-vessel wall interaction. Brit J Haematol 1982; $50: 698$.

Aarts PAMM, Bolhuis PA, Sakariassen KS, Heethaar RM and Sixma JI. Red blood cell size is important for adhersion of blood platelets to artery subendohelium. Blood 1983; 62:214-217.

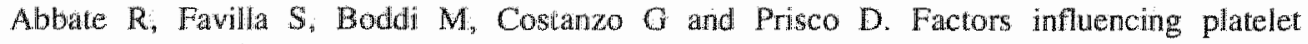
aggregation in whole blood. Am J Clin Pathol 1986;86:91-96.

Abbate R, Boddi M, Prisco D and Gensini GF. Ability of whole blood aggregometer to detect platelet hyperaggregability. Am J Clin Pathol 1989, 91: 159-164.

Akopov S, Grigorian M and Gabrielian E. Effects of drugs acting on platelet and neutrophil aggregation depending on their interaction. Methods Find Exp Clin Pharmacol 1991; 13: 591 594.

Akopov SE. Blood cells interaction and anfiplatelet therapy: A review. Drugs of Today 1992; 28(6): $405-420$.

Antiplatelet Trialists Collaboration. Secondary prevention of vascular disease by prolonged antiplatelet treatment. BMJ 1988; 296: 320-331.

Antiplatelet Trialists Collaboration. Collaborative overview of randomised trials of antiplatelet therapy. BMJ 1994; 308. 81-106, 1.59-168, 235-246.

Bacus JW, Watt $S$ and Trobaugh FE. Clinical evaluation of a new electrical impedance instrument for counting platelets in whole blood. Am J Clin Pathol 1980; 73: 655-663.

Barradas MA, O'Donoghue S, Jagroop A and Mikhailidis DP. Laboratory techniques in drug development (2): Advantages of whole blood platelet aggregation measured by a cell counter (Coulter T-890) in drug evaluation. I Drug Development 1992; 5: $155-166$.

Barstad RM, Orvim U, Hamers MJAG, Roald HE, Tjonnfjord GE, Brosstad F and Sakariassen KS. The geometry of arterial stenoses affects the beneficial effect of aspirin on collageninduced thrombus. Thromb Haenost 1995; 73; 1042 (Abstract).

Baumgartner HR. The role of blood flow in platelet adhesion, fibrin deposition and formation of mural thrombi. Microvasc Res 1973; $5: 167-179$.

Baxter Diagnostics Ine. Detecting abnormalities of primary haemostasis: an in vitro screening
device. Baxter March 1994.

Bertolino $G$, Noris $P$ and Balduini $C L$. Effect of different sample preparation methods on the results of the impedance technique in the study of platelet hyper-and hypo-function in whole blood. Thromb Res 1993; 71(1): 89-94. 
Bevan $J$ and Heptinstall $S$. Serotonin-induced platelet aggregrtion in whole blood and the effects of ketanserin and mepyramine. Thromb Res 1985: 38: 189-194.

Bevers EM, Comfurius $\mathbb{P}$ and Zwaal RFA. Changes in membrane phospholipid distribution during platelet activation. Biochim Biophys Acta 1983; 736: 57-66.

Bick RL. Platelet function defects: A clinical revitw. Semin Thromb Hemost $1992 ; 18(2) ; 167$ 185 .

Blackwell GJ, Flower RJ, Russell-Smith N, Salmon JA, Thorogood PB and Vane IR. Prostacyclin is produced in whole blood. Br J Pharmacol 1978, 64: 436.

Born GVR. Aggregation of blood platelets by adenosine diphosphate and its reversal. Nature $1962 ; 194: 927-929$.

Born GVR. Arterial thrombosis and its prevention. In: Proceedings of the VIll World Congress of Cardiology. Shoji H and Saroru M, eds. Excerpta Medica, Ansterdam, 1979; pp $81-91$.

Burgess-Wilson ME, Cockbill SR, Johnston GI and Heptinstall S. Platelet aggregation in whole blood from patients with Glanzmann's thrombasthenia. Blood $1987 ; 69 ; 38-42$.

Butchers $\mathbb{J}$, Humphrey PPA. Hyde $\mathbb{J}$, Lumley $\mathbb{P}$ and Spurling $\mathrm{NW}$. The ewaluation of a new electronic counting technique for measurement of platelet aggregation in human whole blood in vitro. Br J Pharmacol 1980; 70: 160-161.

Cadroy $\mathrm{Y}$ and Hanson SR. Effects of red blood cell concentration on hemostasis and thrombus formation in a primate model. Blood $1990 ; 75(1)$ ): $2185-2193$.

Cardinal DC and Flower RJ. The electronic aggregometer: a novel device for assessing platelet behaviour in blood. J Pharmacol Methods 1980; 3:135-158.

Cheeseman JE, Mills SP and Hardisty RM. Platelet aggregonetry on whole blood the use of the ELT 8/ds blood cell counter in the investigation of bleeding disorders. Clin Lab Hucmatol $1984 ; 6: 265 \times 272$.

Chen Y-C and Wu KK. A comparison of methods for the study of platelet hyperfimction in thromboembolic disorders. Brit J Haematol 1980; 46: 262-268.

Coller BS. Inherited disorders of platelef function. In: Haemostasis and thrombosis. Bloom AL, Forbes CD, Thomas DP and Tuddenham EGD, eds. Churchill Livingstone, New York, 1994; pp $721-766$.

Cook NS, Zerwes H.G, Tapparelli C, Powling M, Singh J, Mettenich R and Hagenbach A. Platelet aggregation and fibrinogen binding in human, thesus monkey, guincampig, hanster and rat blood: activation by $\mathrm{ADP}$ and a thrombin receptor peptide and inhibition by glycoprotein Ilb/IIa antagonists. Thromb Haemost 1993; $70(3): 531-539$. 
Dey HJ, Young E and Helfrich M. An evaluation of a whole-blood platelet counter. Am I Clin Pathol 1980; 73: 588-593.

De Groot PG and Sixma JJ. Annotation: Platelet Adhesion. Br J Haematol 1990, 75: 308-312.

Diodati $J_{3}$ Quyyumi AA, Hussain $N$ and Keefer LK. Complexes of nitric oxide with nuclcophiles ags agents for the controlled biological release of nitric oxide: antiplatelet effect. Thromb Haemost 1993; 70(4):654-658.

Elwood PC, Beswick AD, Sharp DS. Yarnell WWG, Rogers $S$ and Renaud S. Whole blood impedance platelet aggregometry and ischemic heart disease : The Caerphilly collaborative heart disease study. Arteriosclerosis 1990, 10(6): 1032-1036.

Escolar $G$, Mazzara $R$, Castillo $R$ and Ordinas $A$. The role of the Baumgartner technique in transfusion medicine: research and clinical applications. Transfusion 1994; 34(6): 542-549.

Falcon C. Arnout I and Verrrylen J. Platelet aggregation in whole blood - Studies with a platelet counting technique -. methodological atspects and some applications. Thromb Haemost $1989 ; 61(3): 423-428$.

Flower RJ and Cardinal DC. Use of a novel platelet aggregometer to study the generation by, and actions of, prostacyclin in whole blood. In: Prostacyclin. Vane JR and Bergström S, eds. Raven Press, New York, 1979; pp $211-219$.

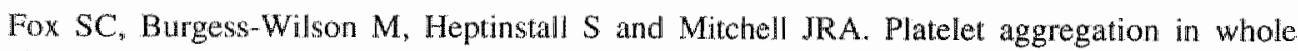
blood determined using the Uitra-Flo 100 platelet counter. Thromb Haemost 1982; 48: 327 329.

Gaarder A, Jonsen I, Laland S, Hel Jem A and Owren PA. Adenosine diphosphate in red cells as. a factor in the adhesiveness of human blood platelets. Nature 1961; 192:531-532.

Goldsmith HL and Turitto VT. Rheological aspects of thrombosis and haemostasis: Basic principles and applications. Thromb Haemost $1986 ; 55(3): 415-435$.

Görög P. A new, ideal technique to monitor thrombolytic therapy. Angiology 1986; 37: 99 . 105.

Gorog DA and Kowacs IB. Shear-induced platelet aggregation has pathological significance through thrombin generation. Thromb Haemost 1995; 73: 1046 (Abstract).

Harrison MVG and Mitchell JRA. The influence of red blood-cells on platelet adhesiveness. Lancet Nov 26 1966; pp |163-1164.

Harrison MJG. Pollock SS and Weisblatt E. Haematocrit and platelet aggregation. Lancet 1984; ii: $9911-992$.

Hendra TJ, Oughton I, Smith CCT, Betteridge DI and Yudkin IS. Exercise-induced changes in platelet aggregation : A comparison of whole blood and platelet rich plasma
techniques.Thromb Res $1988 ; 52: 443451$. 
Hendra TJ and Yudkin IS. Whole blood platelel aggregation based on oell counting procedures. Platelets 1990; 1: 57.66.

Heptinstall S and Taylon PM. The effects of citrate and extracellular calcium ions on the platelet release reaction induced by adenosine diphosphate and collagen. Thromb Hatemost $1979 ; 42: 778-793$.

Hirsh J. Drug therapy: Heparin. N Engl J Med 1991; 324: 1565-1574.

Homstra $G$ and ten Hoor F. The Filtragometer: A new device for measuring platelet aggregation in venous blood of man. Thrombos Diathes Haemorth (Stuttg.) 1975; 34:531544.

Ikeda $Y$, Murata M, Araki Y, Watanabe K, Ando X, llagaki I, Mori $\mathrm{X}$, Ichitani M and Sakai $\mathrm{K}$ Importance of fibrinogen and platelet membrane glycoprotein IVb/ITa in shear-induced aggregation. Thromb Res 1988; 51: 157-163.

Ikeda $\mathrm{Y}$, Handa M, Kawano K, Kamata T, Murata M. Araki Y, Anbo H, Kawai Y, Watanabe $\mathrm{K}$, Itagaki I, Sakai $\mathrm{K}$ and Ruggeri ZM. The role of won Willebrand Factor and fibrinogen in platelet aggregation under varying shear stress. J Clin Invest 1991; 87: 1234-1240.

Ingerman-Wojenski CM, Smith JB and Silver MJ. Evaluation of electrical aggregometry: comparison with optical aggregometry, secretion of ATP, and accumulation of radiolabeled platelets. J Lab Clin Med 1983; 101: 44-52.

Jen $\mathrm{CJ}$ and Mclntire LV. Characturistics of shear-induced aggregation in whole blood. J Lab Clin Med 1984; 103: 115-124.

Jungi TW, Spycher MO, Nydegger UE and Barandun S. Platelet-leukocyte interaction : Selective binding of thrombin-stimulated platelets to human monocytes, polymorphonuclear leukocytes, and related cell lines. Blood 1986; 67: 629-636.

Jügens $\mathrm{R}$ and Braunsteiner H. Zur pathogenase der hrombose. Schweiz Med Wochenschr $1950 ; 80: 1388-1394$.

Kalra J, Mantha SV and Prasad K. Oxygen-free radicals: Key factors in clinical diseases. LabMedica International March/April 1994; pp 16-21.

Karlberg $\mathrm{K}$-E, Chen $\mathbb{E}$, Egberg $\mathbb{N}$ and Sylven C. SIN $\|$ partially and RGDS totally counteracts platelet aggregation as assessed in vitro by two independent whole blood methods. Thromb Res 1993; 72(6): 531-540.

Karpatkin S, Charmatz A. Heterogeneity of human platelets. 1. Metabolic and kinetic evidence suggestive of young and old platelets. J Clin Invest 1969; 48: 1073-1087.

Kessels $H$, Béguin $S$, Andree $H$ and Hemker HC. Measurement of thrombin generation in whole blood - the effect of heparin and aspirin. Thromb Haemost 1994; 72: 78-83. 
Kovacs IB, Hutton RA and Kemoff PBA. Hemostatic evaltuation in bleeding disorders from native blood. Clinical experience with the Hemostatometer. Am J Clin Pathol 1989: 91(3): $271-279$.

Kratzer MAA and Bom GVR. Simulation of primary haemostasis in vitro. Haemostasis 1985: 15: 357-362.

Kroll MH and Schafer Al. Biochemical mechanisms of platelet activation. Blood 1989; 74 : $1181-1195$.

Lowe GDO and Forbes CD. Platelet aggregation, haematocrit, and fibrinogen. Lancet Feb 16 $1985 ;$ pp 395-396.

Ludlam CA. Assessment of platelet function. In: Haemostasis and thrombosis. Bloom AL, Forbes CD, Thomas DP and Tuddenham EGD, eds. Churchill Livingstone, New York, 1994; pp 199-215.

Lumley $P$ and Humphrey PPA. A method for quantitating platelet aggregation and analyzing drug-receptor interactions on platelets in whole blood in vitro. J Pharmacol Methods 1981; 6: $153-166$.

Machi J, Sigel B, Ramos JR, Justin JR, Feinberg H, LeBreton GC and Robertson (Jr) AL. Role of red cells in preventing the growth of platelet aggregation. Thromb Res 1984; 36: 53-66.

Mamman EF. Laboratory measurements of primary haenostasis. Laboratory Hematology 1996: 2(1): 56 (Abstract).

Mann KG, Nesheim ME, Church WR, Haley P and Krishnaswamy S. Surface-dependent reactions of the vitamin K-dependent enzyme complexes. Blood 1990; 76(1): 1-16.

Martin JF, Bath PMW and Burr ML. Influence of platelet size on outcome after myocardial infarction. Lancet 1991; 338: 1409-1411.

Mazeaud MM, Levenson 』, Sang KHLQ. Simon A and Devynck M-A. Platelet aggregation and in vivo shear forces. Thromb Haemost 1994; $71: 26-31$.

MeLaren $M$, Bancroft $A$. Alexander $W$ and Belch JIF. Platelet aggregation in whole blood: comparison between Clay Adams Ultraw FO 100 and Coulter haematology analyser T-540. Platelets 1990: 1: 95-96.

Musumeci $V$, Cremona $G$, Batoni S, Bisbano A, Tutinelli F and Zuppi C. Inhibitory interference of red cells in the measurement of whole blood platelet aggregation by the impedance method. Thromb Res 1987; 45:95-100.

Niewiarowski $S$. Secreted platelet proteins. In: Haemostasis and thrombosis. Bloom AL, Forbes CD, Thomas DP and Tuddenham EGD, eds. Churchill Livingstone, New York, 1994;
pp $167 \% 181$.

O'Brien IR. Some results from a new method of study. J Clin Pathol 1962; 15:452. 
O'Brien JR, Etherington $\mathrm{MD}$ and Weir P. Platelet aggregation inhibitors: a 5 f nuclepore filter 'bleeding time'. Monte Carlo: Siyth international congress on thrombosis, Medittertanean league against thromboembolic disaeses, 1980; 307T.

O'Brien JR. Shear-induced platelet aggregation. Lanced 1990; 335: $711-713$.

Palabrica T, Lobb R, Furie BC, Aronovitz M, Benjamin C. Hsu Y-M, Sajer SA and Furte B. Leukocyte accumulation promoting fibrin deposition is mediated in vivo by P-selectin on adherent platelets. Nature 1992; $359: 848-851$.

Palinski W, Torsellini A and Doni L. Influence of platelet activation on erythrocyte deformability. Thromb Haemost 1983; 49:84-86.

Patscheke $\mathrm{H}$ and Ruf $\mathrm{A}$. The role of platelet aggregation for the shedding of microvesicles. Thromb Haemost 1995; 73: 1002 (Abstract).

Pérez-Requejo JL, Valles J, Santos T and Aznar J. Red cell uptake of serotonin (cl4-5HT): Influence of cellular interactions. Thromb Haemost 1995; 73: 1090 (abstract).

Portele $\mathbb{T}$ and Verhallen PFJ. Simultaneous measurement of platelet-leukocyte adhesion, activation parameter and cell counts by a whole blood flow cytomeny method. Thromb Haemost 1995; 73: 1002 (Abstract).

Ratnoff OD. The development of knowledge about haemostasis and thrombosis. In: Haemostasis and thrombosis. Bloom AL, Forbes CD, Thomas DP and Tuddenham EGD, eds. Churchill Livingstone, New York, 1994; pp 3-28.

Riess $H_{*}$ Braun $G$. Brehm $G$ and Hiller $\mathbb{E}$. Critical evaluation of platelet aggregation in whole human blood. Am J Clin Pathol 1986; 85: 50-56.

Roth GiJ. Deweloping relationships: Arterial platelet adhesion, glycoprotein Ib, and leucine-rich glycoproteins. Blood 1991; 77(1): 5-19.

Ruggeri $Z$ M and Ware $J$. The structure and function of von Willebratid factor. Thromb Haemost 1992; 67(6): 594-599.

Ruggeri ZM. Mechanisms of shear-induced platelet adhesion and aggregation. "Thromb Haemost 1993; 70(1): 119-123.

Russell-Smith NC, Flower RJ and Cardinal DC. Measuring platelet and leucocyle aggregation/adhesion responses in very small volumes of whole blood. I Pharmacol Methods $1981 ; 6: 315-333$.

Salzman EW, Rubino EB and Sims R. Cyclic 3', 5*adensine monophosphate in human blood platelets. III. The role of cyclic AMP in platelet aggregation. Series Haematol 1970; 3: 100$113,358-363$. 
Sanabadi $A R$, Lowe GDO, Forbes $C D$, Prentice $C$ and Barbenel JC. Platelet aggregation studies in whole human blood. Thromb Res 1983; 30:625-632.

Saniabadi AR, Lowe GDO, Barbenel JC and Forbes CD. A comparison of spontaneous platelet aggregation in whole blood with platelet rich plasma: additional evidence for the role of ADP. Thromb Hemosit 1984; 51: 115-118.

Saniabadi AR, Lowe GDO, Barbenel JC and Forbes CD. Further studies on the role of red blood cells in spontancous platelet aggregation. Thromb Res $1985 ; 38: 225-232$.

Saniabadi AR, Lowe GDO, Barbenel JC and Forbes CD. Effect of dipyridamole on spontaneous platelet aggregation in whole blood decreases with the time after venepuncture: evidence for the role of ADP. Thromb Haemost 1987; 58: 744-748.

Shapiro SI and Williams MC. Hemolysis in simple shear flow. Am Inst Chem Eng J 1970; 16: 575-582.

Slack SM, Cui Y and Turitto VT. The effects of flow on blood coagulation and thrombosis. Thromb Haemost 1993; 70(1): $129-134$.

Sloane TE, Blake JW. Disposible blood handling device for measuring haemostasis. US Patent 5047211, Sept 10, 1991.

Splawinski I, Gwozdz B, Ziembicki W, Ziajor M and Splawinska B. Anti-aggregatory activity of $\mathrm{PGI}_{2}$ in whole blood measured by platelet counter. Thromb Res 1984; 34: 93-102.

Splawinska B, Kuzniar $J$ and Splawinska D. Is spontaneous platelet aggregation present in whole blood in vitro. Thromb Haemost 1990; 64(1): 180.

Tangelder G-J, Teirlinck HC, Slaaf DW and Reneman RS. Distribution of blood platelets flowing in arterioles. Am J Physiol 1985; 248: H318-H323.

Tschopp TB. Baumgartner HR, Siberbauer K and Sinzinger H. Platelet adhesion and platelet thrombus formation on subendothelium of human arteries and veins exposed to flowing blood in virro. A comparison with rabbit norta. Haemostasis $1979 ; 8: 19-29$.

Thatulow E, Erikssen I, Sitndwik L, Stomorken H and Cohn PF. Blood platelet count and function are related to total and cardiovascular death in apparently healthy men. Circulation $1991 ; 84: 613-617$

Thompson CB, Eatton KA, Princiotta SM, Rushin CA and Valeri CR. Size dependent platelet subpopulations: relationship of platelet volume to ultrastructure, enzyme activity, and function. Br Maematol 1982; 56:509-519.

Thompson NT, Scrutton MC and Wallis RB. Particle volume changes associated with light transmittance changes in the platelet aggregometer: dependence upon aggregating agent and effectiveness of stimulus. Thromb Res $1986 ; 41: 615-626$. 
Trip MD, Cats VM, van Capelle FJL and Vreeken J. Platelet hyperreativity and prognosis in survivors of myocardial infarction. N Engl J Med 1990; 322(22): 1549-1554.

Turitto VT and Weiss HJ. Red blood cells: their dual role in thrombus formation. Science $1980 ; 207: 541-543$.

Valles I, Santos MT, Marcus AJ, Safier LB, Broekman MJ, Islam N, Ullman HL and Aznar J. Downregulation of human platelet reactivity by neutrophils. I Clin lnvest 1993; 92: 1357-1365.

Violi F, Pratico D, Iuliano L and Balsano F. Dipyridamole potentiates the inhibition of platelet aggregation by aspirin (in human platelet rich plasma and whole blood). J Lipid Mediat 1991; $61-68$.

Warkentin TE and Kelton JG. Acquired platelet disorders. In: Haemostasis and thrombosis. Bloom AL, Forbes CD, Thomas DP and Tuddenham EGD, eds. Churchill Livingstone, New York, 1994; pp 767-815.

Weiss HJ, Hawiger J, Ruggeri ZM, Turitto VT, Thiagarajan $P$ and Hoffmann T. Fibrinogenindependent platelet adhesion and thrombus formation on subendothelium mediated by glycoprotein IIb-IIIa complex at high shear rate. J Clin Invest 1989;83: 288-297.

Willems C, Stel HV, van Aken WG and van Mourik JA. Binding and inactivation of prostacyclin by human erythrocytes. Br J Haematol 1983; 54: 43-52.

Woldhuis B, Tangelder G-J, Slaaf DW and Reneman RS. Concentration profile of blood platelets differs in arteriolles and venules. Am J Physiol 1992; 262: H1217-H1223.

Wu KK, Papp AC, Manner CE and Hall ER Interaction between lymphoctes and platelets in the synthesis of prostacyclin. J Clin Invest 1987; 79: 1601-1606. 


\title{
Platelet aggregation in whole blood determined using the Ultra- Flo 100 platelet counter.
}

Fox SC, Burgess-Wilson M, Heptinstall S and Mitchell JRA. Thromb Haemost $1982,48: 327-329$.

\begin{abstract}
The Ultra-Flo 100 whole blood platelet counter has proved a useful tool for measuring platelet aggregation in whole blood, the extent of aggregation being deduced from the number of single platelets that remain. The technique has shown that platelets aggregate 'spontaneously' in citrated and heparinised blood, but not in blood collected into EDTA. The aggregation occurs during storage but its rate is enhanced by stirring and it occurs more readily when the whole blood has been exposed to plastic rather than to glass. It occurs much more readily in the whole blood of some individuals than from others. It is thought that the process may involve adenosine diphosphate (ADP), possibly leaking from the red cells due to the shear action on them in the stirred sample. The rate of aggregation seen in whole blood is enhanced by several platelet agonists, including collagen, ADP, and sodium arachidonate, which are more usually studied in platelet rich plasma.
\end{abstract}




\section{Introduction}

Most studies of platelet aggregation are carried out in platelet-rich plasma (PRP) which is prepared from anticoagulated blood by removing the bulk of the red and white cells, usually by centrifugation. This is because light transmission techniques for the semi-quantitation of platelet aggregation in PRP are readily available $(1,2)$, whereas techniques for the study of platelet aggregation in whole blood are not as well developed. However, the study of whole blood rather than PRP, offers the attraction that the complete population of platelets could be studied, whereas in PRP a sub-population of hyperaggregatable platelets may be lost during the preparation procedure. In addition, the other cell types present in whole blood could well influence the platelets' behaviour; the red cells can release ADP (3) which is a potent platelet aggregating agent, whilst while cells can release platelet activating factor (4) and prostacyclin, a potent inhibitor of platelet aggregation (5). In this report it will be shown how platelet aggregation can be monitored in whole blood using the Ultra-Flo 100 whole blood platelet counter. Also some of the factors that influence this test parameter will be investigated, and the different patterns of results seen within 'normal' individuals.

\section{Materials and methods}

\section{Materials}

Blood was donated by healthy volunteers. Adenosine 5'-diphosphate sodium salt (ADP), collagen (Type II from bovine achilles tendon), creatine phosphate (CP) and phosphoenolpyruvate (PEP) were obtained from the Sigma Chemical Co. and dissolved in $0.9 \% \mathrm{NaCl}$ before use. The collagen and dissolved with the aid of sonification. Arachidonic acid (grade 1 from porcine liver), creatine phosphokinase (CPK) and pyruvate kinase (PK) were also obtained from the Sigma Chemical Co. The arachidonic acid was converted into the sodium salt by dissolving it in sodium carbonate just before use. Heparin solution containing 5000 IU sodium heparin/ml and $0.15 \%$ chlorocresol as preservative was purchased from Weddel Pharmaceuticals Ltd. Tubes that contained potassium EDTA and were of $4 \mathrm{ml}$ capacity were supplied by Labco Ltd. The $0.9 \%(w / v) \mathrm{NaCl}$ used was prepared by the Boots Chemical Co. for infusion purposes. All other materials were of Analar grade. 


\section{Methods}

Blood Collection: Blood was collected by clean venepuncture into a polypropylene syringe using a 19 gauge $2^{\prime \prime}$ needle. Immediately after collection aliquots were transferred to polystyrene tubes (capacity $10 \mathrm{ml}$ ) that contained either $3.8 \%(\mathrm{w} / \mathrm{v})$ trisodium citrate dihydrate $(\mathrm{ml})$ or $100 \mathrm{lU}$ heparin $(20 \mu 1$ of the stock solution in $180 \mu \mathrm{l}$ of $0.9 \% \mathrm{NaCl}$ ). Aliquots were also transferred to tubes ( $4 \mathrm{ml}$ capacity) that contained $6.4 \mathrm{mg}$ potassium EDTA. The anticoagulated blood obtained by mixing the blood with EDTA, citrate and heparin will be referred to in the report by the abbreviations: $\mathrm{EB}, \mathrm{CB}$ and $\mathrm{HB}$ respectively.

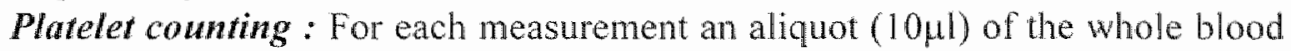
was transferred into a plastic phial that contained $9.1 \mathrm{ml}$ of $0.9 \% \mathrm{NaCl}$. The contents were then mixed and this phial was connected directly to the Ultra-Flo 100 which aspirated the sample and counted the number of platelets. The whole operation takes about 30 seconds. Platelet counts were performed at various intervals after the blood had been collected. They were also performed at intervals after aliquots $(500 \mu \mathrm{l})$ of the whole blood had been stirred in a polystyrene aggregometry tube $\left(3 \mathrm{ml}\right.$ capacity) in a waterbath at $37^{\circ} \mathrm{C}$. The stirring was effected using a metal stirrer bar and a magnetic stirrer operating at $1000 \mathrm{rpm}$. The effect of adding various agents to the whole blood was determined. These agents included: collagen, ADP, sodium arachidonate, $C P$ and $\mathrm{CPK}, \mathrm{PEP}$ and PK. Any changes in the platelet count in $\mathrm{CB}, \mathrm{HB}$ and $\mathrm{EB}$ were usually expressed as a percentage of the platelet count in untreated $\mathrm{EB}$.

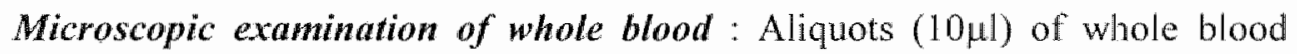
were spread onto clean microscope slides in the form of a blood film, which was dried and then fixed with methanol and stained with MayGrunwald/Giemsa stain. These stained blood films were examined under the microscope at $10 \mathrm{x}$ and $40 \mathrm{x}$ magnification for platelet distribution.

Equipment: The Ultra-Flo 100 whole blood platelet counter was made by Clay Adams Lid. and supplied by Laboratory Impex Ltd. It takes diluted whole blood and uses hydrodynamic focusing to bring the cells into the centre of a counting aperture where they are electronically sized. The number of cells in the size range of red cells and platelets are counted. The machine the calculates the ratio of platelets to red cells. The actual red cell count must be determined separately (in this case on a Coulter counter Model FN) and when this number is provided to the Ultra-Flo 100 it allows the automatic display and printout of the number of platelets in the blood sample. The Ultra-Flo has been fully described and evaluated as a whole blood platelet counter by Bacus et al (6). 
In the present study the Ultra-Flo 100 was used to assess the extent of platelet aggregation in the samples of blood under investigation by monitoring the fall in platelet count in the sample as aggregation occurred. The machine was used according to the maker's instructions except that $0.9 \% \mathrm{NaCl}$ was used in place of the proprietary solution for diluting the whole blood prior to platelet counting because the latter contains EDTA which is known to disaggregate platelets.

\section{Results}

\section{Time-dependent changes in platelet count in whole blood}

When samples of $E B$ were stored at room temperature the number of single platelets remained constant. In contrast, during storage of $\mathrm{CB}$ and $\mathrm{HB}$ at room temperature the number of single platelets in the preparation gradually fell. Figure 1 shows that after two hours in $\mathrm{CB}$ only $84 \%$ of the initial number of single platelets remained. The $\mathrm{pH}$ of the blood samples was measured and showed no change.

\section{Changes in platelet count in stirred whole blood}

When samples of EB were stirred at $37^{\circ} \mathrm{C}$ for up to 8 minutes, there was little change in the single-platelet count. The same result was obtained when other samples of the same blood were examined up to two hours later. However, stirring $C B$ led to a marked fall in the single-platelet count (Figure 2). When samples of the same $\mathrm{CB}$ were stored at room temperature for 60 minutes and then stirred the rate of fall in platelet count remained the same although the starting counts were lower. Similar results were seen with HB.

\section{Microscopic examination of whole blood}

Blood films of EB prepared before and after stirring showed an even distribution of single platelets throughout the slide. Slides of fresh $\mathrm{CB}$ before stirring showed an even distribution of platelets in the film with some platelets together as pairs or triplets. Slides of CB after stirring showed fewer single platelets in the film; aggregates containing many hundreds of cells were observed in the tail of the blood films. The fall in platelet count observed using the Ultra-Flo 100 in stirred $\mathrm{CB}$ is thus correlated with aggregation observed under the microscope. 


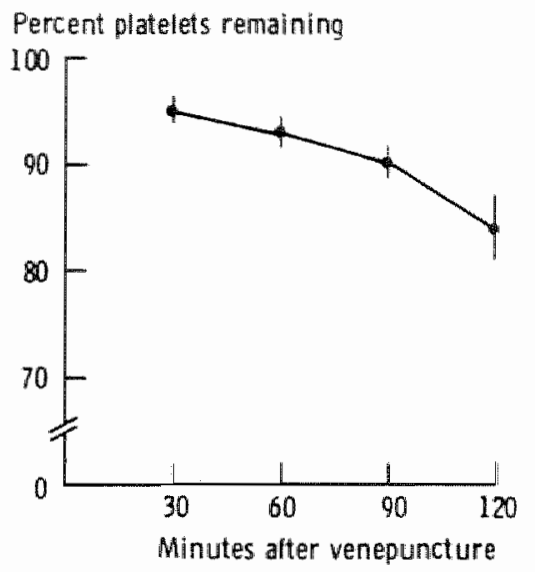

Figure 1

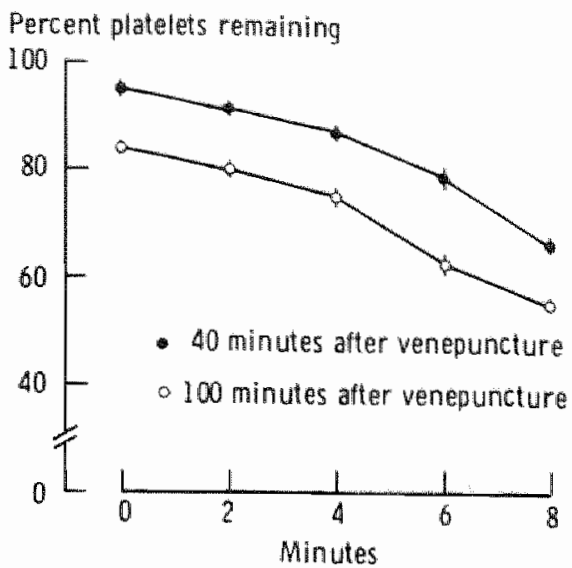

Fig. 1 The number of single platelets remaining in CB (expressed as a percentage of the number in EB) after samples had been stored for different lengths of time at room temperature. The values are the mean ( \pm SEM.) of the results obtained for nine different indiwiduals.

Fig. 2 The number of single platelets remaining in $\mathrm{CB}$ (expressed as a percentage of the number in EB) after stirring samples for different lengths of time at $37^{\circ} \mathrm{C}$. The values are the mean ( $\$$ SEM.) of the results obtained for nine different individuals.

\section{Reproducibility of 'spontaneous" platelet aggregation.}

'Spontaneous' aggregation, defined as a decrease in the number of single platelets, occurred in all samples of $C B$ that we have tested but was more marked in CB from some individuals than others, and this finding was reproducible for the same individual studied on several occasions over a period of several months (Figure 3).

\section{Factors involved in 'spontaneous' platelet aggregation}

a) Surface contact : $\mathrm{CB}$ prepared using a polypropylene syringe and polystyrene collecting and aggregation tubes shows more 'spontaneous' 


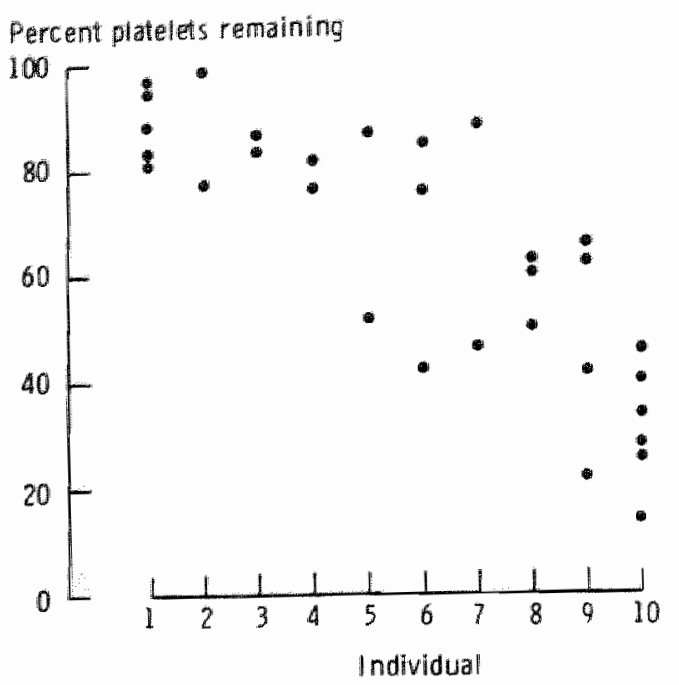

Figure 3

Fig. 3 The number of single platelets remaining in $\mathrm{CB}$ (expressed as a percentage of the number in $\mathrm{EB}$ ) after stirring samples at $37^{\circ} \mathrm{C}$ for six minutes. The results obtained for ten individuals each tested on various occasions are given.

Fig. 4 The number of single platelets remaining in $\mathrm{CB}$ (expressed as a percentage of the number in EB) after stirring samples at $37^{\circ} \mathrm{C}$. The samples had been collected and evaluated using only glass or plastic apparatus. The values are mean (and range) of the results obtained for 3 individuals.

aggregation than CB taken at the same time from the same donor using a glass syringe and glass collecting and aggregation tunes which had been cleaned with chromic acid (Figure 4).

b) ADP: The enzyme substrate systems $\mathrm{PEP} / \mathrm{PK}$ and $\mathrm{CP} / \mathrm{CPK}$ each convert ADP to ATP, so when added to whole blood would be expected to remove, or reduce the level of $\mathrm{ADP}$ from the plasma. Adding either $\mathrm{PEP} / \mathrm{PK}$ or $\mathrm{CP} / \mathrm{CPK}$ to $\mathrm{CB}$ immediately before samples were stirred at $37^{\circ} \mathrm{C}$ inhibited the 'spontaneous' aggregation. The addition of $\mathrm{PK}$ or $\mathrm{CPK}$ alone without substrate also reduced 'spontaneous' aggregation (Figure 5). 


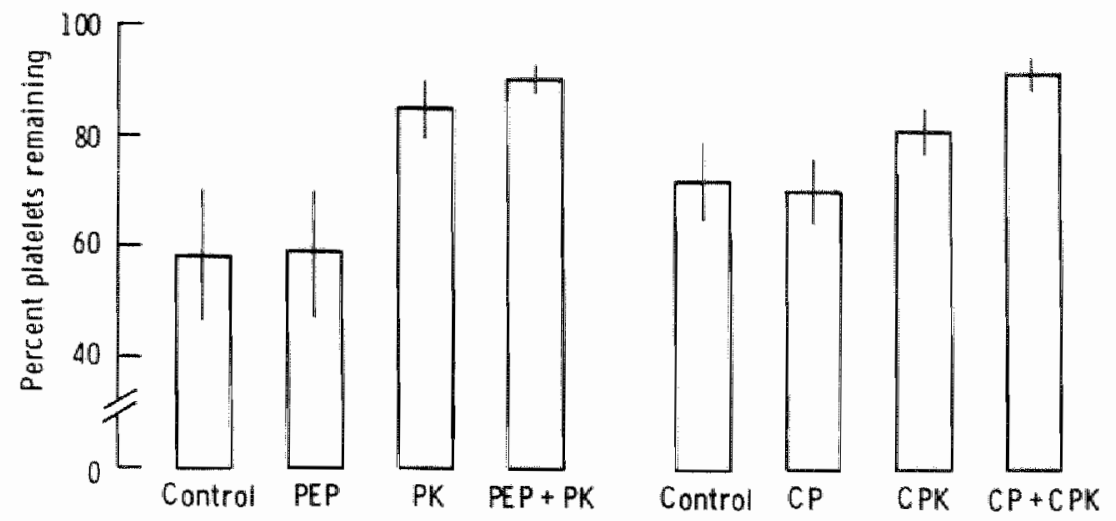

Figure 5

Fig. 5 The number of single platelets remaining in $\mathrm{CB}$ (expressed as a percentage of the number in EB) after stirring samples at $37^{\circ} \mathrm{C}$ for 6 minutes in the presence of PEP (300 M) and/or PK $(15 \mathrm{u})$ or $\mathrm{CP}(300 \mu \mathrm{M})$ and/or CPK $(11 \mathrm{u})$. The values are mean ( $\pm \mathrm{SEM}$ ) of results obtained for three (PEP/PK) and four (CP/CPK) preparations of $\mathrm{CB}$ from different individuals.

\section{Effect of aggregating agents}

Donors who exhibited little 'spontaneous' aggregation in stirred whole blood were chosen. Addition of collagen, ADP and sodium arachidonate increased the rate at which the platelet count fell in both $\mathrm{CB}$ and $\mathrm{HB}$ in a dose-dependent manner (Figure 6). The effects of collagen were irreversible while at some concentrations the effect of ADP was reversible. At some concentrations sodium arachidonate induced an immediate fall in platelet count, while at another, the fall in platelet count followed a short delay. As few as $5 \%$ of the platelets remained when aggregation was maximal. 

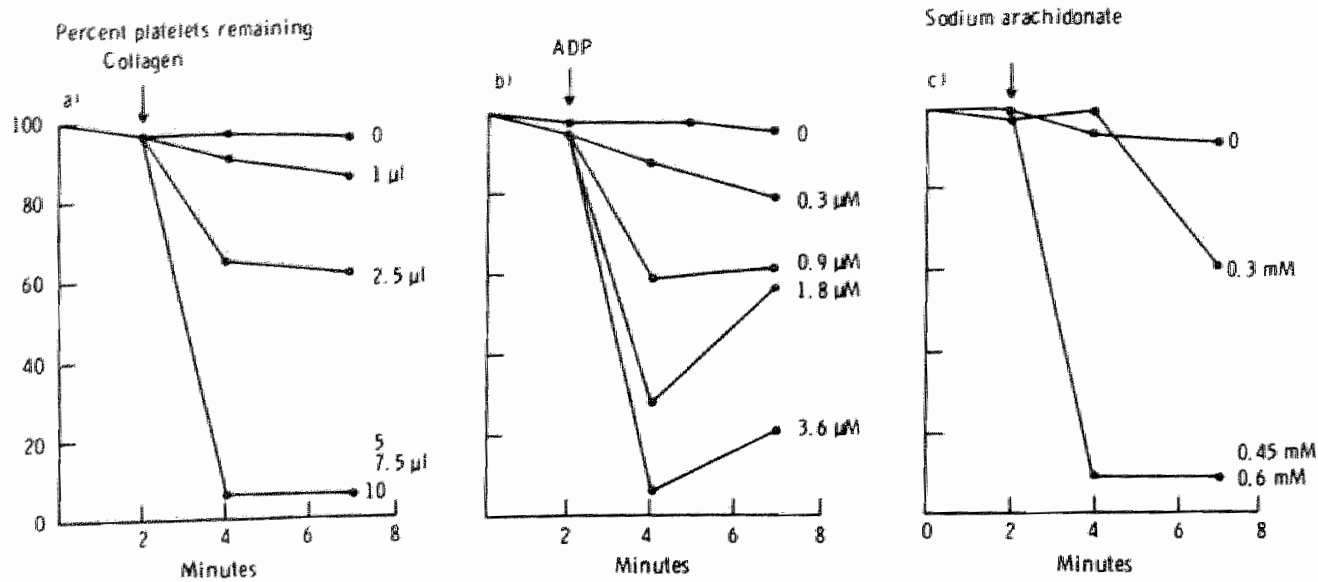

\section{Figure 6}

Fig. 6

The number of single platelets remaining in $\mathrm{CB}$ (expressed as a percentage of the number in EB) after stirring samples at $37^{\circ} \mathrm{C}$ in the presence of a) collagen (1 to $10 \mathrm{\mu l}$ of a collagen preparation), b) $\mathrm{ADP}$ (final concentration 0.3 to $3.8 \mu \mathrm{M}$ in $\mathrm{CB}$ ) and c) sodium arachiclonate $(0.3$ to $0.6 \mathrm{mM}$ in $\mathrm{CB})$. The points at which the agents were added are indicated.

\section{Discussion}

These experiments have shown that the Ultra-Flo 100 is a convenient means of monitoring platelet aggregation in whole blood. They have also shown that platelets in whole blood aggregate spontaneously when the sample is stirred. Other workers have used the Ultra-Flo 100 to monitor platelet aggregation but have not commented on this 'spontaneous' aggregation $(7,8)$.

Platelet aggregates form in citrated and heparinised whole blood during storage, and form more rapidly when the blood is stirred. They do not form when EDTA is present, implying that the aggregation is dependent on the presence of divalent cations. Aggregation is more rapid when the whole blood is exposed to plastic rather than glass. The different rates of 'spontaneous' aggregation in whole blood from different individuals, coupled with the consistency of this observation, implies that its measurement may be of value for the comparative study of platelets from different individuals. It is now important to measure 
'spontaneous' aggregation in whole blood from subjects with, or at risk of, vascular disease as well as in blood from healthy volunteers. Such studies could help explain the presence of "circulating" platelet aggregates in blood from at least some individuals (9) and might give information on whether they are truely "circulating" or formed in vitro.

In an attempt to find out why platelets aggregate 'spontaneously' in stored and stirred whole blood we looked at the effect of the ADP-removing enzyme systems, PEP/PK and $\mathrm{CP} / \mathrm{CPK}$. These abolished the aggregation but $\mathrm{PK}$ and CPK used alone also reduced aggregation. Perhaps the amount of ADP that is required to induce platelets to aggregate 'spontaneously' in whole blood is very small and adequate amounts of the substrates for PK and CPK are available in the plasma.

The platelet aggregation that is induced by agents such as collagen, ADP or sodium arachidonate is usually studied in platelet-rich plasma (PRP). The present experiments have shown however, that the aggregation induced by these agents can also be studied in whole blood. Our preliminary investigations suggest that the changes in the number of single platelets in whole blood that can occur after adding an aggregating agent are remarkably similar to the changes in light transmission that occur when the agent is added to PRP. Collagen induced an apparently irreversible fall in platelet count, the extent of which was dose dependent; it induces similar dose-dependent changes in light transmission (10). The reversible changes in platelet count that occurred in response to some concentrations of ADP are similar to the reversible changes seen in PRP (1). The rapid fall in platelet count seen after adding some concentrations of sodium arachidonate to whole blood, and the delay prior to the fall in platelet count when a lower concentration of sodium arachidonate was used, also reflect the changes in light transmission that occur in PRP. A criticism of studies of platelet aggregation in PRP is that large clumps of platelets that are visible to the naked eye contain many hundreds of cells need to form before changes in light transmission can occur. This criticism cannot be levelled at the present technique and it focuses only on the single platelets that remain, and we consider that further studies of platelet aggregation in whole blood using the Ultra-Flo 100 are warranted. 


\section{References}

1. Born GVR. Aggregation of blood platelets by adenosine diphosphate and its reversal. Nature (Lond) 1962, 194:927-929.

2. Adams J, Heptinstall S, Mitchell JRA. A six channel automated platelet aggregometer. Thrombos Diathes Haemorrh 1975, 34:821-824.

3. Gaarder A, Jonsen J, Laland $\$$, Hellem AJ, Owren PA. Adenosine diphosphate in red cells as a factor in the adhesiveness of human blood platelets. Nature 1961, 192: 531532 .

4. Benveniste J, Cochrane $\mathrm{CG}_{3}$ Henson PM. Leukocyte-dependent histamine release from rabbit platelets: the role of IgE, basophils and platelet-activating factor. J Exp Med $1972,136: 1356-1377$.

5. Blackwell GJ, Flower RJ, Russell-Smith N, Salmon JA, Thorogood PB and Vane JR. Prostacyclin is produced in whole blood. Br J Pharmacol 1978, 64: 436.

6. Bacus IW, Watt S, Trobaugh FE. Clinical evaluation of a new electrical impedance instrument for counting platelets in whole blood. Am J Clin Pathol 1980, 73: 655-663.

7. Butchers I, Humphrey PPA, Hyde J, Lumley P and Spurling NW. The evaluation of a new electronic counting technique for measurement of platelet aggregation in human whole blood in vitro. Br J Pharmacol 1980, 70:160-161.

8. Nunn B. Comparison of the effects of aspirin in humans ex vivo in platelet-rich plasma and whole blood. Thromb Haemostas $1981,46: 50$ (Abst.).

9. Wu KK, Hoak JC. A new method for the quantitative detection of platelet aggregates in patients with arterial insufficiency. Lancet 1974, 2: 924-927.

10. Heptinstall S, Taylor PM. The effects of citrate and extracellular calcium ions on the platelet relase reaction induced by adenosine diphosphate and collagen. Thromb Haemostas $1979,42: 778-793$. 


\title{
CHAPTER 3
}

\section{Spontaneous platelet aggregation in heparinised blood during pregnancy.}

Burgess-Wilson ME, Morrison R and Heptinstall S. Thromb Res 1986, 37: $385-$ 393.

\begin{abstract}
A marked increase in spontaneous platelet aggregation (SPA) in whole blood was found during pregnancy. The increased spontaneous aggregation was most evident in whole blood anticoagulated with heparin; it was less marked in blood that contained citrate as anticoagulant. Studies of blood that contained both anticoagulants indicated that it is heparin that potentiates aggregation in blood taken during pregnancy rather than citrate that inhibits it. Increased SPA was seen in normotensive pregnancy, in pregnancy complicated by essential hypertension and in pregnancy-induced hypertension. In normotensive pregnancy it was evident at 16 weeks gestation but in pregnancy complicated with essential hypertension it was not evident until 24 weeks gestation. For all the women SPA had returned to nomal six weeks after delivery.
\end{abstract}




\section{Introduction}

There are reports that platelets in citrated platelet rich plasma (PRP) prepared from blood from pregnant women are more sensitive to aggregating agents $(1,2)$ and are less sensitive to the inhibitory effects of prostacyclin $(3,4)$. When we attempted to confirm and extend these observations we found that the PRP prepared from blood that had been anticoagulated with heparin often contained very few platelets compared with PRP from blood that had been anticoagulated with citrate, or compared with heparinised PRP from non-pregnant subjects. To explore the reasons for this we have measured spontameous platelet aggregation (SPA) in samples of heparinised whole blood and citrated whole blood taken from women during pregnancy. We have compared the results with the values obtained for the same women six weeks after delivery, and with those obtained for non-pregnant female controls. We have also compared the results obtained during normal pregnancy, pregnancy complicated with essential hypertension (EHT) and pregnancy-induced hypertension (PIH).

\section{Materials and methods}

\section{Subjects}

Forty-six pregnant women and ten non-pregnant women took part in the study. Fourteen of the pregnant women remained normotensive throughout the study, eleven presented with EHT (blood pressure in early pregnancy $>140 / 85 \mathrm{~mm}$ $\mathrm{Hg}$ ), and 21 women were enrolled when they developed PIH in the third trimester of pregnancy. PIH was diagnosed, if a previously normotensive women developed a blood pressure of $>140 / 90 \mathrm{~mm} \mathrm{Hg}$ on two occasions separated by more than 24 hours. The mean ages of the normotensive, EHT and PIH groups were 28,30 and 26 years respectively, and the mean age of the nonpregnant controls was 27 years. None of the non-pregnant controls were taking oral contraceptives.

Venous blood samples were taken from the normotensive women and those with EHT at 16,24, 32 and 36 weeks gestation and then six weeks after delivery. For the women with PIH one blood sample was taken in the third trimester and another six weeks after delivery. Only one sample was taken from the non-pregnant controls.

Blood was taken from a further six women to study the effect of the combination of citrate and heparin on SPA. Three were normotensive, pregnant 
women who were between 32 and 36 weeks gestation and three were agematched non-pregnant controls.

\section{Materials}

The heparin that we used was bactericide-free heparin injection B.P. (Monoparin) from Weddel Pharmaceuticals Limited, supplied at a concentration of 5000 units $/ \mathrm{ml}$. The solution that was used to anticoagulate blood was a 1 in 10 dilution of the stock solution in saline (infusible saline from Travenol Laboratories). The solution of citrate that was used to anticoagulate blood was $3.8 \%(\mathrm{w} / \mathrm{v})$ trisodium citrate dihydrate. Tubes $(4 \mathrm{ml}$ capacity) that contained 6.4 mg of potassium EDTA were obtained from Labco Limited.

\section{Blood collection}

Blood was collected with minimum venous stasis from the antecubital vein into polypropylene syringes using a 19 gauge, 2 inch needle. Care was taken to ensure that minimal local trauma was caused by the venepuncture. In cases where there was difficulty in drawing the blood the sample was discarded. Aliquots were dispensed into two polystyrene tubes ( $10 \mathrm{ml}$ capacity), one that contained heparin ( $200 \mu \mathrm{l}$, final concentration $10 \mathrm{units} / \mathrm{ml}$ of blood) and another that contained citrate $(1 \mathrm{ml})$. A further aliquot of blood $(4 \mathrm{ml})$ was dispensed into a tube that contained EDTA, and another aliquot $(10 \mathrm{ml})$ was dispensed into an empty glass tube in which the blood was allowed to clot.

The $10 \mathrm{~m} \|$ samples of heparinised blood (HB) and citrated blood (CB) were used to measure SPA. After the platelet studies had been performed the remaining $\mathrm{CB}$ was centrifuged at $1200 \mathrm{~g}$ for 15 minutes at room temperature and the supernatant plasma removed and stored at $-50^{\circ} \mathrm{C}$. The plasma was later assayed for fibrinogen and albumin. The EDTA blood was used to measure the white cell count, platelet count and haematocrit. The clotted blood sample was used to measure the urate level.

To study the effects of the combination of heparin and citrate on SPA, two $10 \mathrm{ml}$ samples of $\mathrm{HB}$ and $\mathrm{CB}$ were obtained as described above. Exactly five minutes after venepuncture heparin $(200 \mu l)$ was added to one of the samples of $C B$, and citrate $(\mathrm{Iml})$ was added to one of the samples of HB. 


\section{Spontancous platelet aggregation (SPA)}

SPA was always measured between 30 and 40 minutes after venepuncture. In the interim the samples were allowed to stand at room temperature. SPA was assessed by counting the number of single platelets in samples taken from whole blood that was stirred at $37^{\circ} \mathrm{C}(6)$. Blood samples (400ul) were placed in polystyrene tubes and stirred at $1000 \mathrm{rpm}$ using stirrer bars that were $4 \mathrm{~mm}$ lengths of stainless steel wire ( $1 \mathrm{~mm}$ diameter). Care was taken to avoid the formation of bubbles and it was noted that no settling of the sample occurred during testing. Aliquots $(10 \mu l)$ were removed before stirring and every two minutes thereafter up to eight minutes. In most experiments the number of platelets in these samples were determined immediately using the Ultra-Flo 100 whole blood platelet counter as previously described (5). In the experiments in which the effect of the combination of heparin and citrate was measured the samples were fixed prior to platelet counting (6). The extent of SPA was expressed as the percentage fall in the number of single platelets in the preparation compared with the number of platelets in a sample of blood collected into EDTA.

\section{Plasma proteins and other haematological indices}

The method described by Clauss (7) was used to measure thrombin-clottable fibrinogen. The albumin levels were measured using the technique described by Doumas and Biggs (8). The white cell count, platelet count and haematocrit were determined using a Coulter Counter (model S+IV). Urate was determined using the Urica Color Kit supplied by Boehringer Mannheim.

\section{Statisrical analysis}

The Student t-test was used for group comparisons. 


\section{Results}

\section{Spontaneous Platelet Aggregation (SPA)}

Six weeks after delivery SPA in HB was very similar to that in the non-pregnant controls (figure 1). However, during pregnancy, SPA was markedly increased. In normotensive pregnancy the increase in SPA was already evident at 16 weeks gestation ( $p<0.01$ c.f. post-natal), but was most pronounced between 24 and 36 weeks. In EHT, SPA in HB was also increased but in this case it was not until 24 weeks that a significant difference between the value obtained during pregnancy and the value obtained six weeks after delivery was evident $(p<0.001)$. In PIH, SPA in the third trimester was similar to that in normotensive pregnancy and in EHT. Results obtained for patients with mild PIH (urate level of $400 \mu \mathrm{mol} / 1$ and below, $n=12$ ) and those with more severe $\mathrm{PIH}$ (urate levels in excess of $400 \mu \mathrm{mol} / 1, \mathrm{n}=9$ ) were not significantly different.

Normotensive Pregnancy

EHT
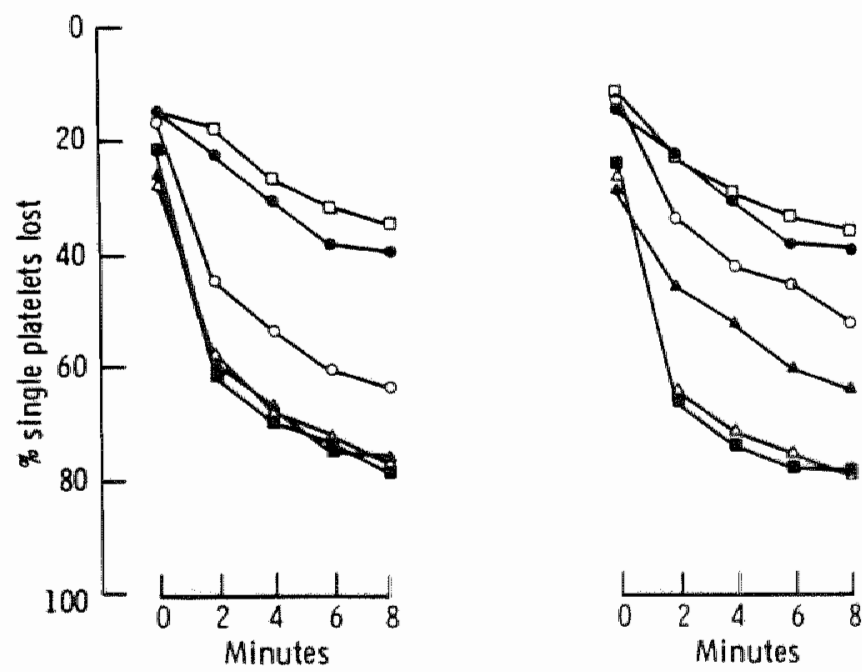

Figure I

Fig. 1 SPA in HB during normotensive pregnancy and EHT. Blood samples were tested at 16 $(0), 24(\Delta), 32(\Delta)$ and $36(\mathbf{D})$ weeks gestation, and a six weeks post-natal ( $\square)$. Also shown are results obtained for the controls $(\bullet)$. 
In $\mathrm{CB}$ any changes in SPA in normotensive pregnancy, EHT and PIH were less marked than those in $\mathrm{HB}$ (figure 2). In normotensive pregnancy the SPA in samples at 16 and 24 weeks gestation was increased compared with the postnatal sample $(p<0.01)$. In EHT the SPA in the sample taken at 32 weeks gestation was increased compared with the post-natal sample $(p<0.02)$. When citrate was added to HB taken from either late-pregnant or non-pregnant female subjects there was no change in the extent of SPA seen (figure 3). When heparin was added to $C B$ from non-pregnant female subjects, again there was no significant change in SPA. However, when heparin was added to $C B$ from latepregnant subjects there was a marked increase in SPA $(p<0.05$ c.f. citrated blood).
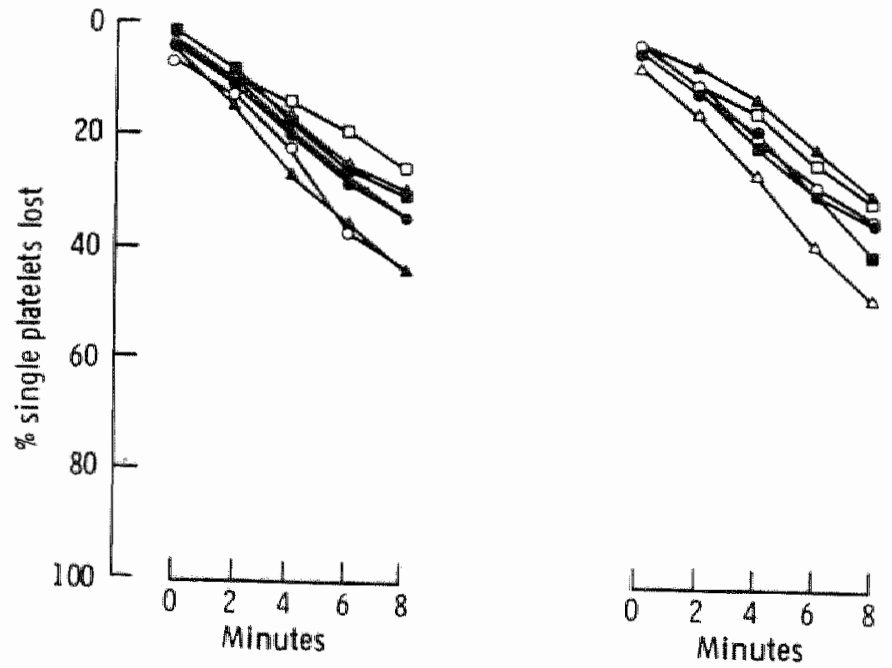

\section{Figure 2}

Fig. 2 SPA in CB during nomotensive pregnancy and EHT. Blood samples were tested at 16

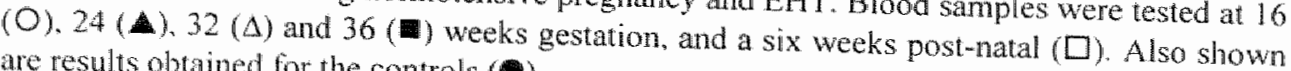
are results obtained for the controls ( ). 


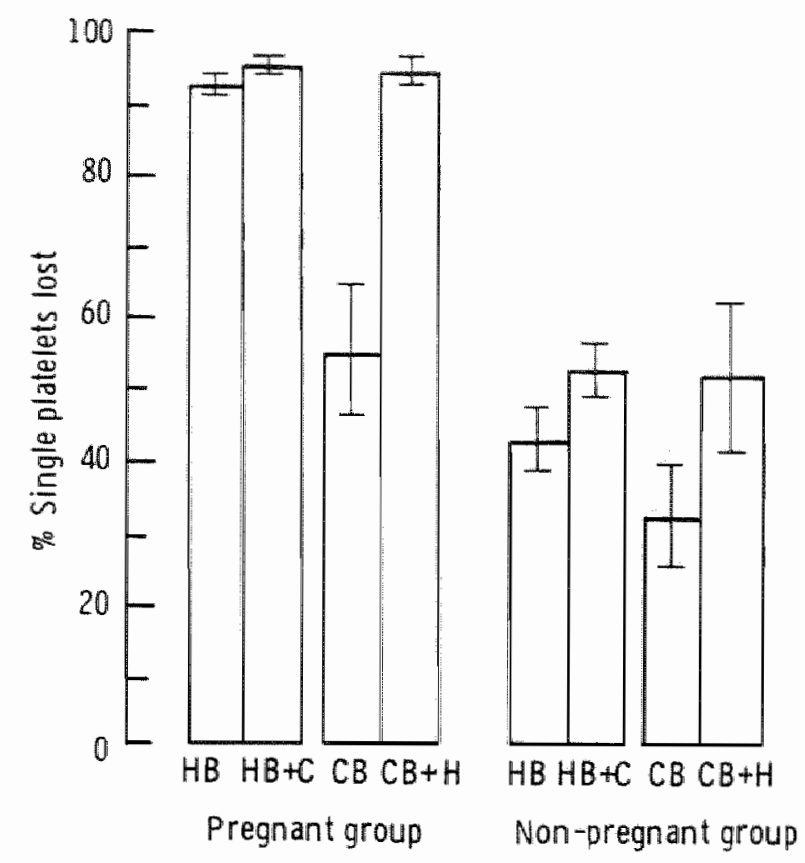

Figure 3

Fig. 3 SPA in $\mathrm{HB}, \mathrm{HB}$ to which citrate had been added $(\mathrm{HB}+\mathrm{C}), \mathrm{CB}$ and $\mathrm{CB}$ to which heparin had been added $(\mathrm{CB}+\mathrm{H})$. Samples were obtained from three pregnant women and three non-pregnant controls. Values are the percentage of platelets lost after stirring the blood for 8 minutes (mean \pm SEM).

\section{Plasma proteins and other haematological indices}

All the results that were obtained in normotensive pregnancy and EHT are given in table 1. Results obtained in PIH were similar to those obtained in EHT. The changes seen in plasma proteins and other haematological indices were similar to those seen in previous investigations of pregnancy (9-12). An additional observation was that the plasma fibrinogen levels were higher in EHT than in normotensive pregnancy. 


\section{Table 1}

Changes in fibrinogen, Albumin, White cell count, Platelet count and Haematocrit during Pregnancy.

\begin{tabular}{|c|c|c|c|c|c|c|c|c|c|c|c|}
\hline \multirow[t]{3}{*}{ Indices } & \multicolumn{10}{|c|}{ Gestation (weeks) } & \multirow[t]{3}{*}{ Controls } \\
\hline & \multicolumn{2}{|c|}{16} & \multicolumn{2}{|c|}{24} & \multicolumn{2}{|c|}{32} & \multicolumn{2}{|c|}{36} & \multicolumn{2}{|c|}{$6 \mathrm{PN}$} & \\
\hline & $\mathrm{NT}$ & $\mathrm{EHT}$ & NT & EHT & $\overline{\mathrm{NT}}$ & $\mathrm{EHT}$ & NT & EH' & $\mathrm{NT}$ & EHT & \\
\hline & & & & & & & & & & & \\
\hline Fibrimogen & 315 & 364 & 332 & 370 & 341 & 412 & 410 & 442 & 233 & 272 & 252 \\
\hline$(\mathrm{mg} / \mathrm{d})$ & \pm 18 & \pm 29 & \pm 16 & \pm 29 & \pm 20 & \pm 26 & \pm 21 & \pm 33 & \pm 15 & \pm 17 & 417 \\
\hline & & & & & & & & & & & \\
\hline Albumin & 32 & 34 & 31 & 37 & 31 & 34 & 30 & 34 & 36 & 38 & 37 \\
\hline$(\mu \mathrm{mol} \|)$ & \pm 1 & \pm 1 & \pm 1 & \pm 1 & \pm 1 & \pm 1 & \pm 1 & \pm 1 & \pm 1 & \pm 1 & \pm 1 \\
\hline WCC & & & & & & & & & & & \\
\hline$\frac{w c c}{\left(\times 10^{y} / 1\right)}$ & 8.0 & 9.5 & 9.5 & 9.1 & 8.6 & 9.4 & 9.2 & 8.9 & 5.9 & 6.6 & 6.1 \\
\hline$(x \mid 0 y, 1)$ & \pm 0.4 & \pm 0.8 & \pm 0.6 & \pm 0.7 & \pm 0.4 & \pm 0.6 & \pm 0.6 & \pm 0.5 & \pm 0.4 & \pm 0.6 & \pm 0.5 \\
\hline $\mathrm{PC}$ & 229 & & & & & 647 & $7 \%$ & & & & \\
\hline$\left(\times 10^{\circ} / 1\right)$ & & 231 & 226 & 222 & 203 & 213 & 213 & 204 & 247 & 246 & 261 \\
\hline & & \pm 29 & \pm 13 & \pm 20 & \pm 12 & \pm 23 & \pm 20 & \pm 16 & \pm 14 & \pm 20 & \pm 18 \\
\hline Haematocrit & 37 & 37 & 34 & 33 & 35 & 33 & 35 & 36 & 37 & 40 & 38 \\
\hline$(\%)$ & \pm 1 & \pm 1 & \pm 1 & \pm 1 & \pm 1 & 12 & \pm 1 & \pm 1 & \pm 1 & \pm 1 & \pm 1 \\
\hline & & & & & & & & & & & \\
\hline
\end{tabular}

Abbreviations: $\mathrm{NT}$ - nomotensive pregnancy, EHT - essential hypertension in pregnancy, $6 \mathrm{PN}-6$ weeks post natal, WCC - white cell count, PC - platelet count. Results are mean $上$ SEM.

\section{Discussion and conclusions}

These experiments show that SPA is markedly increased in HB during pregnancy; in CB the increase in SPA is less marked. The results of the experiments in which both anticoagulants were added to whole blood suggest that it is likely that heparin potentiates platelet aggregation in blood taken during pregnancy, rather than citrate inhibits it. The increased rate of SPA in 
HB was evident in normal pregnancy, in EHT and in PIH. In normotensive pregnancy it was evident at 16 weeks gestation but in EHT it did not become significant untill later in pregnancy.

In a study of SPA in normal, non-pregnant subjects (13) we found that age and haematocrit were independent determinants of $\mathrm{SPA}$ in both $\mathrm{HB}$ and $\mathrm{CB}$ and that there is also a relationship between SPA and fibrinogen and between SPA and platelet count. In the present study, care was taken to ensure that any differences in SPA could not be due to differences in the age of the individuals in the groups studied. As the haematocrit and platelet count are reduced in pregnancy it is unlikely that these indices could account for the increased SPA. Plasma fibrinogen levels increased during pregnancy but the pattern of this change did not match the pattern obtained for SPA. Moreover, in the present study increased SPA was only observed in HB during pregnancy whereas in our previous study the relationship that we observed between SPA and fibrinogen was evident in both $\mathrm{HB}$ and $\mathrm{CB}$. In addition, fibrinogen levels were higher in EHT than in normotensive pregnancy yet the increased SPA was evident earlier in normotensive pregnancy than in EHT. We consider it unlikely, therefore, that increased SPA in pregnancy is a consequence of increased fibrinogen levels.

It has long been recognised that in some circumstances heparin and heparin-like substances can cause platelet aggregation and release of platelet granule contents $(14,15)$, and the existence of a plasma component that acts as a cofactor for the aggregation has been postulated (16). Whether the increased platelet aggregation in $\mathrm{HB}$ during pregnancy is due to an increased level of this co-factor, or is a reflection of increased sensitivity of platelets to aggregatory effects of heparin is unknown. It is believed that SPA in whole blood is a consequence of ADP being liberated from red cells $(5,13,17)$. Perhaps heparin enhances the rate at which ADP is liberated.

These experiments may explain the reduced platelet counts found in PRP prepared from HB taken during pregnancy. They suggest that heparin enhances platelet aggregation so that during the centrifugation that is required to prepare PRP the platelets aggregates would enter the red cell layer and would be removed from the PRP. It is possible that the platelets remaining in PRP prepared from $\mathrm{HB}$ taken during pregnancy are a hypoaggregable subfraction of the original platelet population and are not representative of that original population. Thus care should be taken in extrapolating data obtained using heparinised PRP in this condition. 
The observation that platelets aggregate much more readily in the presence of heparin during pregnancy than in the non-pregnant state may have implications for the use of heparin in the thromboembolic conditions that can occur during pregnancy. Clearly, further studies to assess the possible deleterious consequences of heparin administration during pregnancy are desirable.

\section{Acknowledgments}

We are grateful for the technical assistance of Miss $J$ Crawford and Miss M Hawkins. We thank the Department of Haematology, University Hospital, Nottingham for measuring the haematocrit, platelet count and white cell counts, and the Department of Clinical Chemistry for the albumin and urate analyses. This work was partly supported by a grant from CORDA.

\section{References}

1. Lewis PJ, Boylan P, Friedman LA, Hensby CN and Downing 1. Prostacyclin in pregnancy. Br Med J 1980; 280; 1581-1582.

2. Andrews NP, Broughton Pipkin F and Heptinstall S. Blood platelet behaviour in mothers and neonates. Thromb Haemostas 1985; 53: 428-432.

3. Hoche C, Kellalides A, Dadad C and Sinzinger H. Platelet sensitivity to prostacyclin in pregnancy and puerperium. In: Prostacyclin in pregnancy. P.J.Lewis, ed. Raven Press, New York, 1983, pp 189-193.

4. Briel RC and Lippert TH. Platelet sensitivity to prostacyclin in normal and complicated pregnancy. In: Prostacyclin in pregnancy. P.J.Lewis, ed. Raven Press, New York, 1983, p 195-197.

5. Fox SC, Burgess-Wilson M, Heptinstall $\mathrm{S}$ and Mitchell JRA. Platelet aggregation in whole blood determined using the Ultra-Flo 100 platelet counter. Thromb Haemostas $1982 ; 48: 327-329$.

6. Bevan $\mathbb{J}$ and Heptinstall $\mathrm{S}$. Serotonin-induced platelet aggregation in whole blood and the effect of Ketanserin and Mepyramine. Thromb Res 1985; 38: 189-194. 
7. Clauss Von A. Gerimmngphysiologische schellmethode zur bestimmung des fibrinogens. Acta Haematol 1957; 17:237-247.

8. Doumas, B.T. and Biggs H.G. Detemination of serum albumin. In: Standard melhods of clinical chemistry. G.R.Cooper, ed. Acadenic Press Ine. Nev York, 1972, pp 175.

9. Tooke JE and Nicol GP. Thrombotic disorders associated with pregnancy and the pill. Clinics in Haematol $1981 ; 10: 613-630$.

10. Howie PW, Prentice CRM and MoNicol GP. Coagulation, fibrinolysis and platelet function in pre-eclampsia, essential hypertension and placental insufficiency. I Obster Gynaecol 1971; 78: 992-1003.

11. Gilabert J, Aznar J, Parrilla JJ, Reganon E, Vila V and Estelles A. Alterations in the coaguation and fibrinolysis system in pregnancy, labour and puerperium, with special reference to a possible transitory state of intravascular coagulation during labour. Thromb Haemostas $1978 ; 40: 387-396$.

12. Howie PW. Blood clotting and fibrinolysis in pregnancy. Postgrad Med J 1979; 55 : $362-366$.

13. Burgess-Wilson ME, Green S, Heptinstall S and Mitchell JRA. Spontaneous platelet aggregation in whole blood: dependance on age and haematocrit. Lancet 1984; ii: 1213.

14. Eika $\mathrm{C}$. On the mechanism of platelet aggregation induced by heparin, protanine and polybrene. Scand J Haematol 1972; 9: 248-257.

15. Thomson C, Forbes CD and Prentice CRM. The potentiation of platelet aggregation and adhesion by heparin in vitro and in vivo. Clin Sci Mol Med 1973; 45: $485-494$.

16. Salzman EW, Rosenberg RD, Smith MH, Lindon JN and Favreau L. Effect of heparin and heparin fractions on platelet aggregation. J Clin Invest $1980 ; 65: 64-73$.

17. Saniabadi AR, Lowe GDO, Barbenel IC and Forbes CD A comparison of spontaneous platelet aggregation in whole blood with platelet rich plasma Additional evidence for the role of ADP. Thromb Haemostas 1984;51:115-118. 


\title{
CHAPTER 4
}

\section{Further study of heparin-induced whole blood platelet aggregation.}

\begin{abstract}
We had previously observed that the extent of heparin-induced whole blood platelet aggregation (HIA) was much greater in blood samples collected from pregnant subject when compared with age/sex matched controls. Further study suggests the existence of an undefined plasma 'factor' which facilitates HIA. We observed that the extent of HIA observed in the whole blood from one individual could be modified by the addition of plasma from another individual. It was possible to inhibit HIA with substances that are known to inhibit platelet activation, e.g. apyrase and prostacyclin. The extent of HIA was found to vary considerably between different normal individuals, being greater in normal females than in normal males. The extent of HIA is a measurement of the sensitivity of the blood to heparin, and it could be quantified by measuring the extent of whole blood platelet aggregation induced using a range of heparin concentrations added to citrated whole blood. Further studies are needed to investigate the clinical utility of this technique.
\end{abstract}




\section{Introduction}

Heparin can be used to inhibit clotting in vivo and in vitro. It can also induce platelet aggregation in vivo and in vitro. Heparin-induced thrombocytopenia (HIT) has been recognised for many years, and is one of the most important drug reactions occurring today. It is quite common, and is seen in between $2-10 \%$ of the patients who receive heparin therapy. Heparin-induced platelet aggregation (HIA) is a phenomenon that may be observed when platelets are exposed to heparin in vitro. This has led to the development of several laboratory tests designed to detect HIA (Salam and Van der Weyden 1983, Kelton et al 1984, Sheridan et al 1986, Greinacher et al 1991, Kelton 1994). These tests of in vitro HIA are designed to identify individuals at risk of developing HIT. The link between the HIA observed in vitro and the clinical complication of HIT has not yet, however, been clarified.

Systematic studies of platelets have shown that heparin can specifically potentiate platelet aggregation in PRP, gel-filtered, washed and formaldehyde-fixed platelet preparations (Saba et al 1984). Isolated platelets were shown to behave quite differently from the platelets suspended in PRP. It was suggested that the activity of a plasma 'factor' produces HIA and that this activity might vary in different individuals, and might be altered during different pathological states. When studying blood samples from pregnant women marked HIA was observed, the extent of which increased during pregnancy (Burgess-Wilson et al 1986). When the same women were tested 6 weeks after delivery they did not differ from age/sex matched controls. Blockmans et al (1986) showed that a 'factor' that is present in the plasma can cause HIA in some cases of HIT.

In this study we describe a test using whole blood platelet aggregation that can be used to detect and quantify HA in vitro. The principle of the test is based on the possibility that a plasma 'factor' of some type is responsible for the HIA. We have investigated the mechanism of the effect and ways of inhibiting it.

\section{General methods}

Sample collection. Blood was collected as described previously (Burgess-Wilson et al 1986), into EDTA and citrate as anticoagulants. An extra $10 \mathrm{ml}$ citrate sample was collected for the preparation of plasma for storage.

Plasma preparation and storage. Samples of plasma were prepared by centrifuging a $10 \mathrm{ml}$ sample of citrated blood at $1200 \mathrm{~g}$ for 15 minutes at room 
temperature. The supernatant plasma was removed and stored at $-50^{\circ} \mathrm{C}$. Prior to use, plasma was thawed out by incubation in a $37^{\circ} \mathrm{C}$ waterbath for 5 minutes.

Blood grouping. The $\mathrm{ABO}$ and Rhesus D typing of each subject tested was performed by the Transfusion laboratory, Haematology Department, University Hospital, Nottingham. In the experiments where plasma from one individual was added to a whole blood sample from another individual care was taken to use $\mathrm{ABO}$ and Rhesus $\mathrm{D}$ compatible donors.

Whole blood aggregation studies. When not otherwise specified the whole blood aggregation testing was performed using the methods described previously by Bevan and Heptinstall 1985. Specific method details are described in the following method and results section.

\section{Materials}

The heparin stock solution was heparin injection B.P. (Monoparin) from Weddel Pharmaceuticals Limited, supplied at a concentration of 5000 units $/ \mathrm{ml}$. The solution that was used to anticoagulate blood was a 1 in 10 dilution of the stock solution in $0.9 \%$ saline. The solution of citrate that was used to anticoagulate blood was $3.8 \%(\mathrm{w} / \mathrm{v})$ trisodium citrate dihydrate. Tubes $(4 \mathrm{ml}$ capacity) that contained $6.4 \mathrm{mg}$ of potassium EDTA were obtained from Labco Limited.

Adenosine 5'-diphosphate (ADP, sodium salt) adrenaline, apyrase (Grade 1), prostacyclin, aspirin and arachidonic acid (AA, grade 1 from porcine liver) were obtained from Sigma Chemical (St. Louis). ADP, adrenaline, apyrase, aspirin and prostacyclin were dissolved in saline before use; AA was converted to the sodium salt before use by dissolving in $0.1 \mathrm{~mol} / \mathrm{l}$ of sodium carbonate. Collagen (suspended equine collagen fibrils) was obtained from Hormon-Chemie and used as directed. The $0.9 \%(w / v) ~ N a C l$ used was prepared by the Boots Chemical $\mathrm{Co}$. for infusion purposes. The dipyridamole was supplied by Boehringer Ingelheim. And the UK 37248 (a thromboxane synthetase inhibitor) was supplied by Pfizer. All other materials were of Analar grade. 


\section{A) Investigation of the mechanism of HIA in vitro}

\section{Methods}

i) The addition of plasma samples to whole blood and heparin

Aliquots (100 II) of stored citrated plasma collected from pregnant $(n=4)$ and nonpregnant females $(n=4)$ was added to $400 \mu$ l of citrated whole blood freshly collected from a non-pregnant female. The mixture had $20 \mu$ l of a heparin solution added giving a final concentration of $10 \mathrm{~J} / \mathrm{ml}$. The tube was then gently mixed and then incubated without further mixing at room temperature for 30 minutes. The mixture was tested using the 'fixing method' described previously (Bevan and Heptinstall 1985) to measure platelet aggregation. Platelet loss was measured only after 8 minutes stirring.

ii) The effect of incubating mixtures of plasma, whole blood and heparin In another experiment, the same methodology was used as previously (i), but the samples of plasma from pregnant subjects $(n=3)$ were incubated unstirred at room temperature with whole blood from a non-pregnant female and heparin for increasing lengths of time. As previously the samples were then stirred for 8 minutes; and the $\%$ of platelets lost determined.

\section{iii) The effect of heat-treating plasma samples}

In the third experiment, citrated plasmas from pregnant females $(n=5)$ were divided into two portions; one portion was heated for 20 minutes at $56^{\circ} \mathrm{C}$, the other stored at room temperature. The heat-treated plasmas were then centrifuged

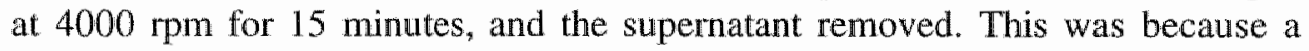
small amount of precipitation appeared, which was removed by the centrifugation. The heat-ireated and non heat-treated citrated plasmas were then added to the citrated whole blood samples from a non-pregnant subject using the previous method (i), and incubated for 30 minutes at room temperature. As previously the samples then were stirred for 8 minutes, and the $\%$ of platelets lost determined.

\section{Results}

i) The addition of plasma samples to whole blood and heparin

Table 1 shows the extent of whole blood platelet aggregation (\% platelets lost after 8 minutes stirring) after plasma from pregnant subjects and non-pregnant females was added to whole blood from a non pregnant female. The extent of platelet aggregation was significantly higher $(p=0.016)$ when plasma from pregnant subjects was added. 


\section{Table 1 Platelet loss $(\%)$ in pregnant and non-pregnant females}

\begin{tabular}{|c|c|c|}
\hline Subject & Pregnant & Non-pregnant \\
\hline 1 & 47 & 26 \\
2 & 70 & 43 \\
3 & 44 & 31 \\
4 & 61 & 24 \\
\hline Mean & $\mathbf{5 6}$ & $\mathbf{3 1}$ \\
SEM & 6.1 & 4.3 \\
\hline
\end{tabular}

ii) The effect of incubating mixtures of plasma, whole blood and heparin Figure 1 shows the effect of incubating the adding plasma samples from pregnant subjects to whole blood from a non-pregnant female and incubating the mixture (unstirred) with heparin for increasing lengths of time. The samples were again stirred for 8 minutes, and the \% of platelets lost determined.

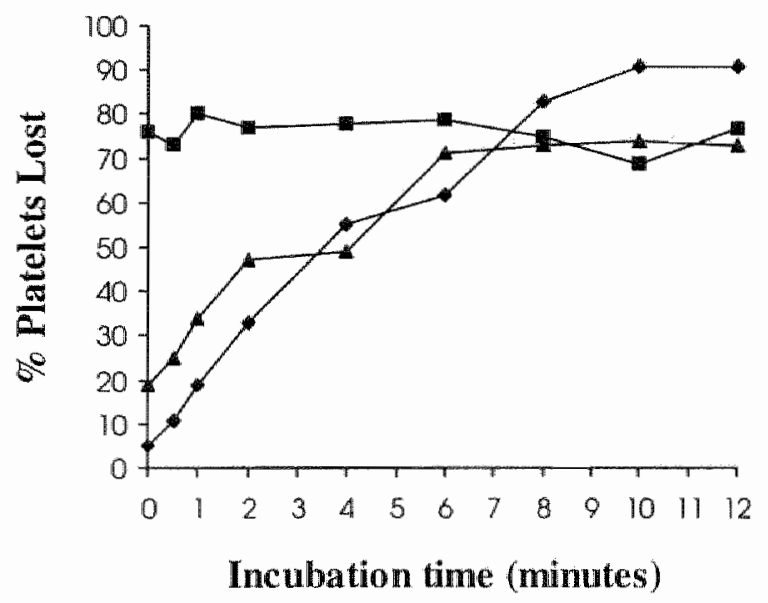

\section{Figure I}

Fig. 1 The time course over which HIA developed when blood was incubated unstirred at room temperature. Plasma from three different pregnant subjects was used; Subjects $\mathrm{A}(\mathbf{-}), \mathrm{B}(\mathbf{A})$, and $\mathrm{C}(\mathbf{H})$.

iii) The effect of heat-treating plasma samples

Table 2 shows the results from the plasmas (heat-treated or none heat-treated). There was no significant difference in the results obtained with heat-treated or none heat-treated plasma. 


\section{Table 2 The effect of heat-treatment (\% platelet loss)}

\begin{tabular}{|c|c|c|}
\hline Subject & $\begin{array}{c}\text { None heat- } \\
\text { treated }\end{array}$ & Heat-ireated \\
\hline 1 & 57 & 52 \\
2 & 66 & 76 \\
3 & 23 & 24 \\
4 & 65 & 62 \\
5 & 53 & 59 \\
\hline Mean & $\mathbf{5 3}$ & $\mathbf{5 5}$ \\
SEM & 8.8 & 9.6 \\
\hline
\end{tabular}

\section{B) A quantitative method for measuring HIA in vitro}

\section{Method}

In this experiment the effect of adding a range of heparin concentrations to citrated whole blood was investigated. A stock solution of heparin $(5000 \mathrm{U} / \mathrm{ml})$ was diluted and $50 \mu \mathrm{l}$ of each dilution was added to 10 plastic tubes, so that when 450 ul of whole blood was added the mixtures would contain final heparin concentrations of : $0.001,0.003,0.01,0.03,0.1,0.3,1,3,10$ and $30 \mathrm{U} / \mathrm{ml}$ respectively. A control tube (zero heparin) containing only $50 \mu \mathrm{l}$ saline was also prepared. A $20 \mathrm{ml}$ citrated blood sample and a $4 \mathrm{ml}$ EDTA sample were collected, and immediately $450 \mu \mathrm{l}$ of citrated whole blood was added to each of the 11 tubes. Each was mixed thoroughly without creating any bubbles and stored at room temperature. At exactly 30 minutes after collection the set of 11 tubes containing citrated blood plus different heparin concentrations were mixed, and then stired for 12 minutes, after which the platelet count was determined. The fixing method of measuring whole blood aggregation was used (Bevan and Heptinstall 1985). This testing (called the HIA Threshold testing) was performed on four different subject groups;

i) normotensive pregnant females between 24 to 37 weeks gestation ( $n=11$ )

ii) non-pregnant normotensive females between $14-50$ years old $(n=9)$

iii) normotensive males between 14-50 years old $(n=6)$

iv) hypertensive males $(\mathrm{n}=3$ )

Results. The mean results for each group are shown in Figure 2. The \% platelet loss at each concentration of heparin are plotted as a heparin threshold profile curve. The extent of SPA is shown where the heparin concentration was zero. 


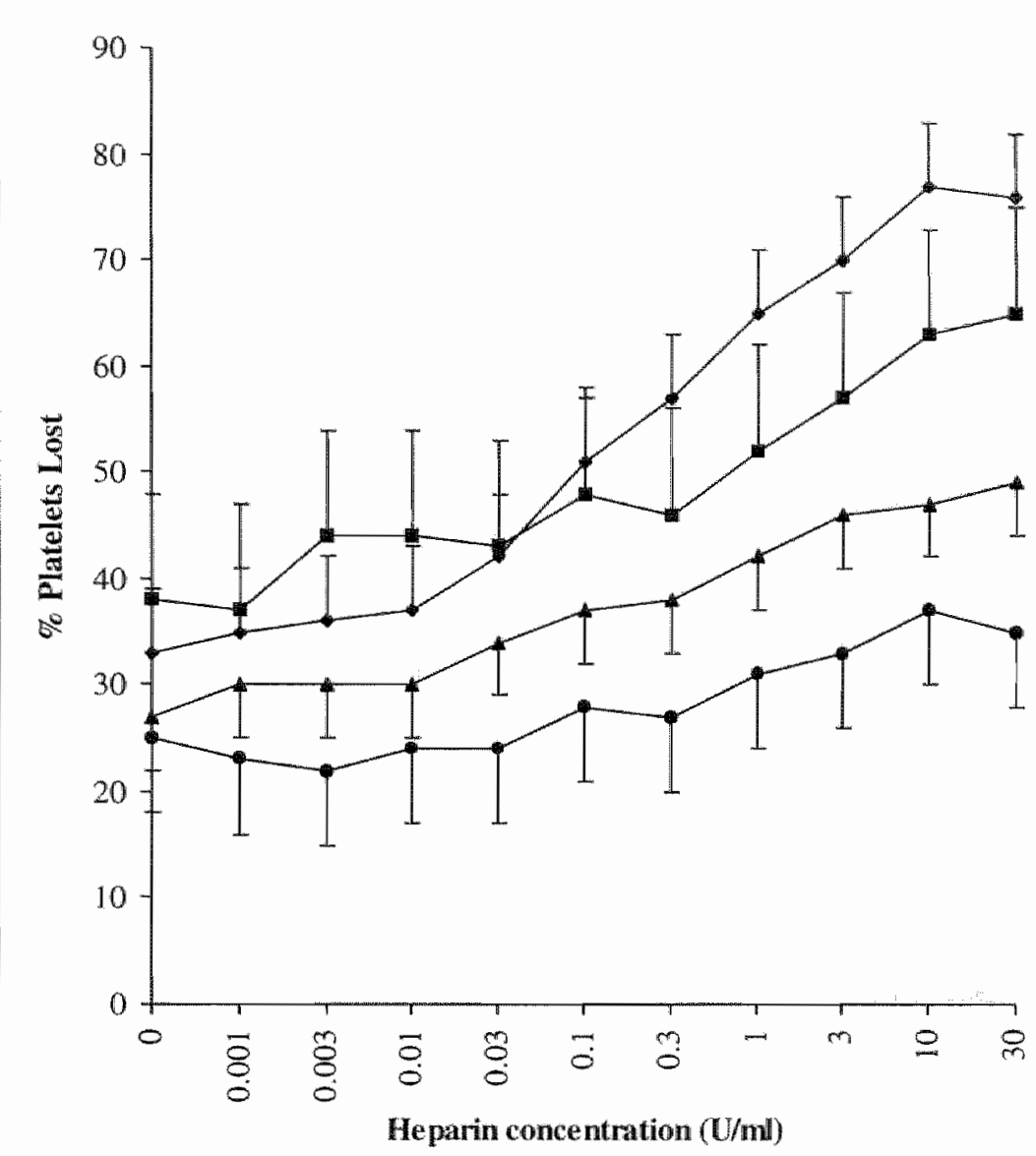

\section{Figure 2}

Fig. 2 The mean $\%$ platelets lost (with the SEM) is shown for the different groups of subjects; pregnant females $(n=11)$ ( ); non-pregnant females $(n=9)(\mathbf{A})$; normotensive males $(n=6)(-)$; and hypertensive males $(n=3)(\mathbf{v})$.

Threshold definition. The 'HIA threshold' was calculated after making the following arbitrary definition: "HIA threshold equals the heparin concentration where an increase of $10 \%$ loss compared with loss due to saline addition was observed". The HIA threshold was calculated for each individual subject and these results are shown in Table 3. 
Table 3 HIA Thresholds for individual subjects

\begin{tabular}{|c|c|c|c|c|}
\hline $\begin{array}{c}\text { HL Threshold } \\
\text { Range }\end{array}$ & $\begin{array}{c}\text { Pregnant } \\
\text { females }\end{array}$ & $\begin{array}{c}\text { Non-pregnant } \\
\text { females }\end{array}$ & $\begin{array}{c}\text { Normotensive } \\
\text { Males }\end{array}$ & $\begin{array}{c}\text { Hypertensive } \\
\text { males }\end{array}$ \\
\hline $0.003-0-03$ & 4 & 2 & 0 & 0 \\
\hline $0.1-1$ & 6 & 4 & 3 & 1 \\
\hline $3-10$ & 1 & 3 & 3 & 2 \\
\hline
\end{tabular}

Other data. The mean age, platelet count, red cell count and $\%$ platelets lost with $10 \mathrm{U} / \mathrm{ml}$ heparin are summarized in Table 4 . The only significant differences found were between the pregnant females and the other three groups. The pregnant females are younger and have a lower red cell count (which is normal for pregnancy), and higher \% platelet loss with $10 \mathrm{U} / \mathrm{ml}$ heparin.

Table 4 Other test parameters

\begin{tabular}{|c|c|c|c|c|}
\hline Parameter & $\begin{array}{c}\text { Pregnant } \\
\text { females }\end{array}$ & $\begin{array}{c}\text { Non- } \\
\text { Pregnant } \\
\text { female }\end{array}$ & $\begin{array}{c}\text { Normo- } \\
\text { tensive } \\
\text { males }\end{array}$ & $\begin{array}{c}\text { Hyper- } \\
\text { tensive } \\
\text { Males }\end{array}$ \\
\hline $\mathrm{n}=$ & 11 & 9 & 6 & 3 \\
\hline Age (years $)$ & 26 & 33 & 37 & 48 \\
\hline Platelet count $\left(\times 10^{9} / \mathrm{l}\right)$ & 277 & 276 & 245 & 228 \\
\hline Red Cell count $\left(\times 10^{12} /\right)$ & 3.83 & 4.54 & 4.91 & 5.50 \\
\hline Platelets lost $(10 \mathrm{U} / \mathrm{ml}$ Heparin) & 77 & 47 & 37 & 63 \\
\hline
\end{tabular}




\section{C) The effect of anti-platelet agents on HIA in vitro}

\section{Methods}

i) The in vitro effect of several platelet inhibitors on whole blood aggregation in the presence and absence of heparin

In this experiment we investigated the in vitro effect of the inhibitors on stirring induced aggregation (which is called elsewhere, "spontaneous platelet aggregation" or SPA), and on heparin-induced aggregation (HIA) in response to two concentrations of heparin. The change in the extent of whole blood aggregation induced by the addition of plasma from three pregnant subjects to whole blood from a non-pregnant female was measured. Citrated whole blood $(400 \mu 1)$ from a non-pregnant female was aliquoted into plastic tubes that contained $20 \mu \mathrm{l}$ of one of the following inhibitors to give final concentrations of; dipyridamole $\left(10^{-4} \mathrm{M}\right)$, prostacyclin $(0.5 \mathrm{ng} / \mathrm{ml})$, UK37248 $\left(10^{-4} \mathrm{M}\right)$, apyrase (1 $\mathrm{U} / \mathrm{ml})$ or aspirin $\left(10^{-4} \mathrm{M}\right)$. The tubes were mixed and allowed to stand at room temperature for 30 minutes. A $100 \mu$ aliquot of stored plasma from a pregnant subject was then added. Then for each inhibitor $20 \mu$ of either saline (no heparin), or heparin $(1 \mathrm{U} / \mathrm{ml}$ or $10 \mathrm{U} / \mathrm{ml})$ was added to a tube. The tubes were then gently mixed and incubated at room temperature for a further 10 minutes. The mixture was tested using the 'fixing method' (Bevan and Heptinstall 1985) to measure platelet aggregation. The $\%$ platelet loss after 8 minutes of stirring was calculated.

\section{ii) The in vitro effect of dipyridamole with and without aspirin on HIA}

Another experiment investigated specifically the effect of dipyridamole in the presence and absence of aspirin. Blood samples were collected into citrate from ten pregnant subjects between $24-36$ weeks gestation. The citrated blood was aliquoted (440 1 ) into two sets of plastic tubes that contained a $20 \mu l$ alliquot of heparin (giving a final concentration $10 \mathrm{U} / \mathrm{ml}$ ), then $20 \mu \mathrm{l}$ of dipyridamole was added (to give final concentrations of $0,1,3,10$ and $30 \mu \mathrm{M}$ ). Into one set of tubes a $20 \mu \mathrm{l}$ aliquot of aspirin (giving a final concentration $100 \mu \mathrm{M}$ ) was added, and into the other set a $20 \mu \mathrm{l}$ aliquot of saline. The tubes were mixed carefully and allowed to stand at room temperature for 30 minutes. The mixture was tested using the 'fixing method' (Bevan and Heptinstall 1985) to measure platelet aggregation. The \% platelet loss after 8 minutes stirring was calculated. 


\section{Results}

i) The in vitro effect of several platelet inhibitors on whole blood aggregation in the presence and absence of heparin

The mean results are shown in figure 3 , which shows the effect of the inhibitors aggregation in the presence of saline (SPA), $1 \mathrm{U} / \mathrm{ml}$ heparin and $10 \mathrm{U} / \mathrm{ml}$ heparin

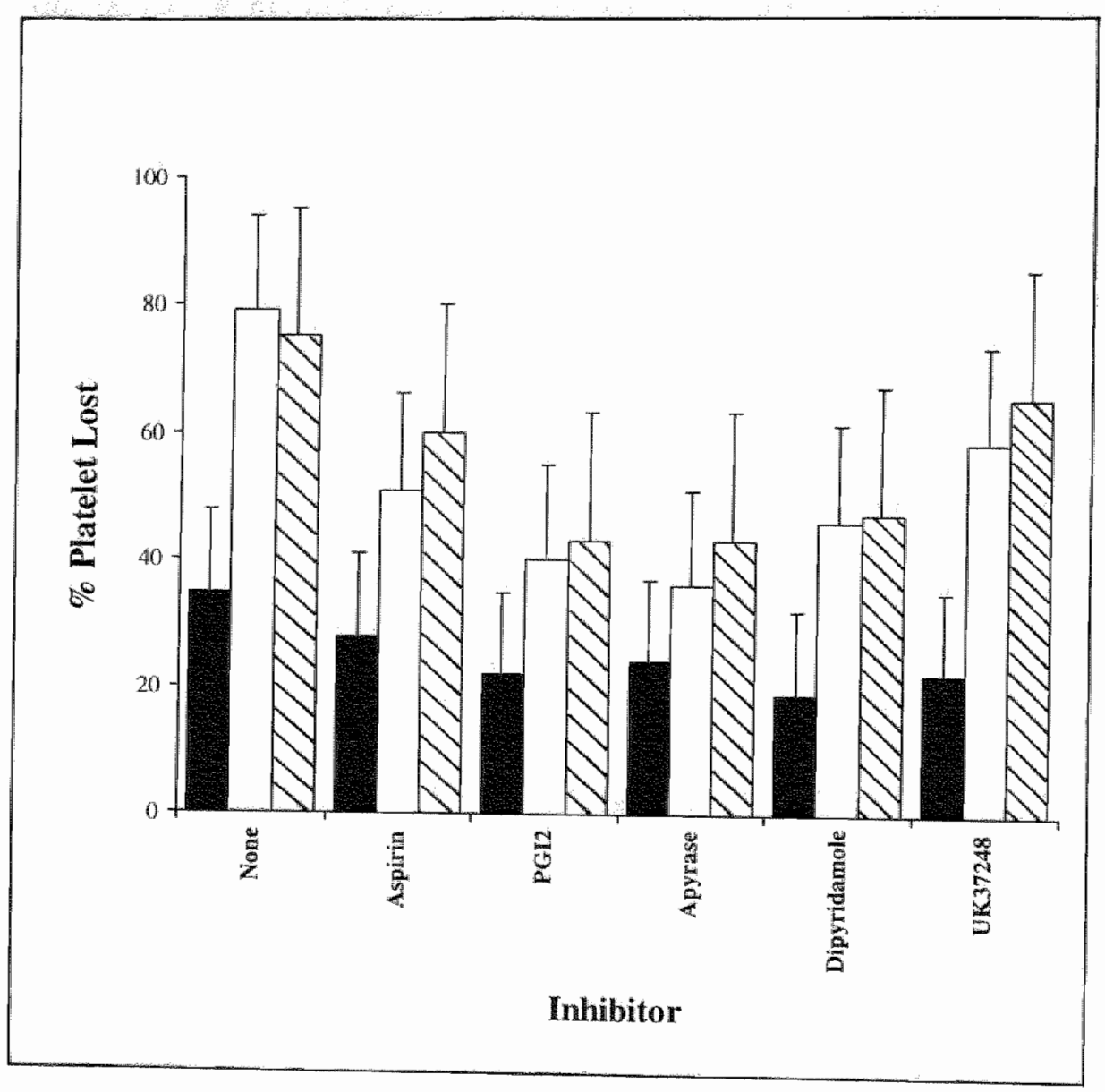

\section{Figure 3}

Fig. 3 The results are the mean \% platelets lost (with SEM) from three experiments. They show the effect of adding different inhibitors, when no heparin is present (solid bars), and when HIA has been induced by $1 \mathrm{U} / \mathrm{ml}$ heparin (open bars) and $10 \mathrm{U} / \mathrm{ml}$ heparin (light shaded bars). Inhibitor concentrations were; aspirin $10^{-4} \mathrm{M}$, prostacyclin $0.5 \mathrm{ng} / \mathrm{ml}$, apyrase $1 \mathrm{U} / \mathrm{ml}$, 
(where the data includes SPA and HIA). With these inhibitor concentrations significant inhibition $(p<0.05)$ was seen only with apyrase and prostacyclin $\left(\mathrm{PGI}_{2}\right)$. Apyrase removes $\mathrm{ADP}$ whilst $\mathrm{PGI}_{2}$ is an adenylate cyclase inhibitor. Aspirin and dipyridamole caused some reduction in \% platelets lost, but the extent of the loss was not statistically significant. UK37248, a thromboxane synthetase inhibitor, appeared to have little effect on the \% platelets lost.

\section{ii) The in vitro effect of dipyridamole with and without aspirin on HLA}

Figure 4 shows the mean results for the 10 subjects. There was a significant reduction in $\%$ platelet loss with $10 \mu \mathrm{M}(\mathrm{p}<0.01)$ and $30 \mu \mathrm{M}$ dipyridamole $(p<0.001)$. Aspirin further reduced the extent of aggregation by about $20 \%$ in the presence and absence of dipyridamole.

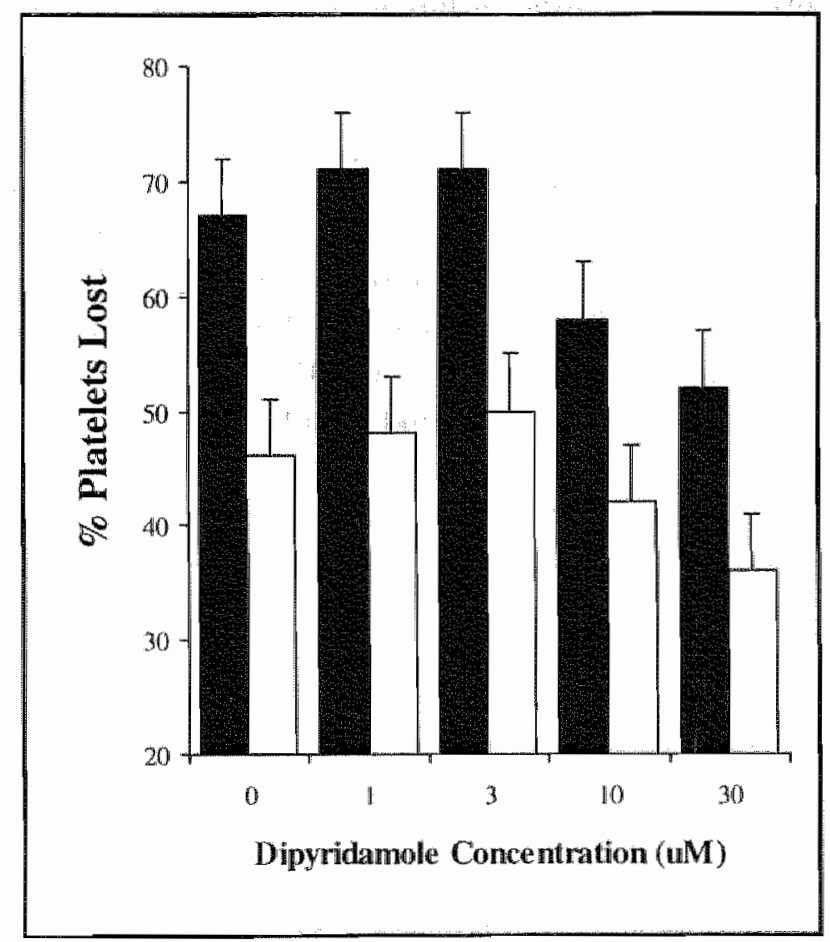

\section{Figure 4}

Fig. 4 The results shown are the mean $\%$ platelet loss plus the SEM. The effect of dipyridamole on HIA is seen on samples for heparinised blood from pregnant subjects ( $n=10$ ), in the presence (open bars) and absence of $100 \mu \mathrm{M}$ aspirin (solid bars). 


\section{D) The effect of aspirin on HIA in an $e x$ vivo study}

\section{Methods}

HIA Thresholds. Aspirin was ingested by 5 normotensive pregnant women, who were day-patients under the care of Department of Obstetrics and Gynaecology, Queens Medical Centre, University Hospital Nottingham. Informed consent was obtained. The mean term was 30 weeks, and the mean age 25 years. Citrated blood samples were obtained just prior to aspirin ingestion (orally, one $75 \mathrm{mg}$ tablet) and approximately 1 hour post ingestion. HIA threshold measured as previously described (see section B). Note that the platelet count was measured after 8 minutes stirring.

Agonist-induced aggregation. The platelet aggregation in citrated blood in response to various agonists was also measured to confirm the effect of aspirin. In these experiments there are some differences in the method compared with the previous experiment. Due to practical considerations, the citrated blood (480 $\mu 1$ ) samples were tested between 10 and 20 minutes after collection (which is sooner than normal). The samples were stirred for 2 minutes, the aggregating agent ( $20 \mu$ 1) was added, and then after only 1 and 2 minutes of stirring (usually samples were stirred for 8 minutes) the platelet count was measured using the fixing technique (21). This can be done because agonist-induced aggregation occurs rapidly, and platelet loss can easily be measured after 1 to 2 minutes of stirring. The method used to calculate the results was different. Instead of using the EDTA platelet count as $100 \%$, the citrated blood platelet count after 2 minutes stirring just prior to adding the agonist was used as the $100 \%$. This calculation method removes the SPA and makes it a measurement of only agonist-induced aggregation.

\section{Results}

HIA Thresholds. The mean results (plus the SEM) before and after the aspirin ingestion can be seen in Figure 5. The post-aspirin ingestion results show a significant reduction $(\mathrm{p}<0.05)$ in the extent of spontaneous aggregation (SPA) in citrated blood (mean $=25 \%)$ compared with pre-aspirin ingestion results (mean $=38 \%$ ). The HIA thresholds, calculated using the previous criteria, increased significantly $(p<0.05)$ from a mean of $0.76 \mathrm{U} / \mathrm{ml}$ pre-aspirin ingestion to $1.88 \mathrm{U} / \mathrm{ml}$ post aspirin ingestion. This suggests that aspirin only reduced the SPA and not the HIA. 
Agonist-induced aggregation. The mean results (plus the SEM) for the 5 subjects are shown in Figure 6. Arachidonic acid (AA) induced platelet aggregation was reduced. Aggregation induced by ADP and collagen, and SPA were reduced, but not significantly.

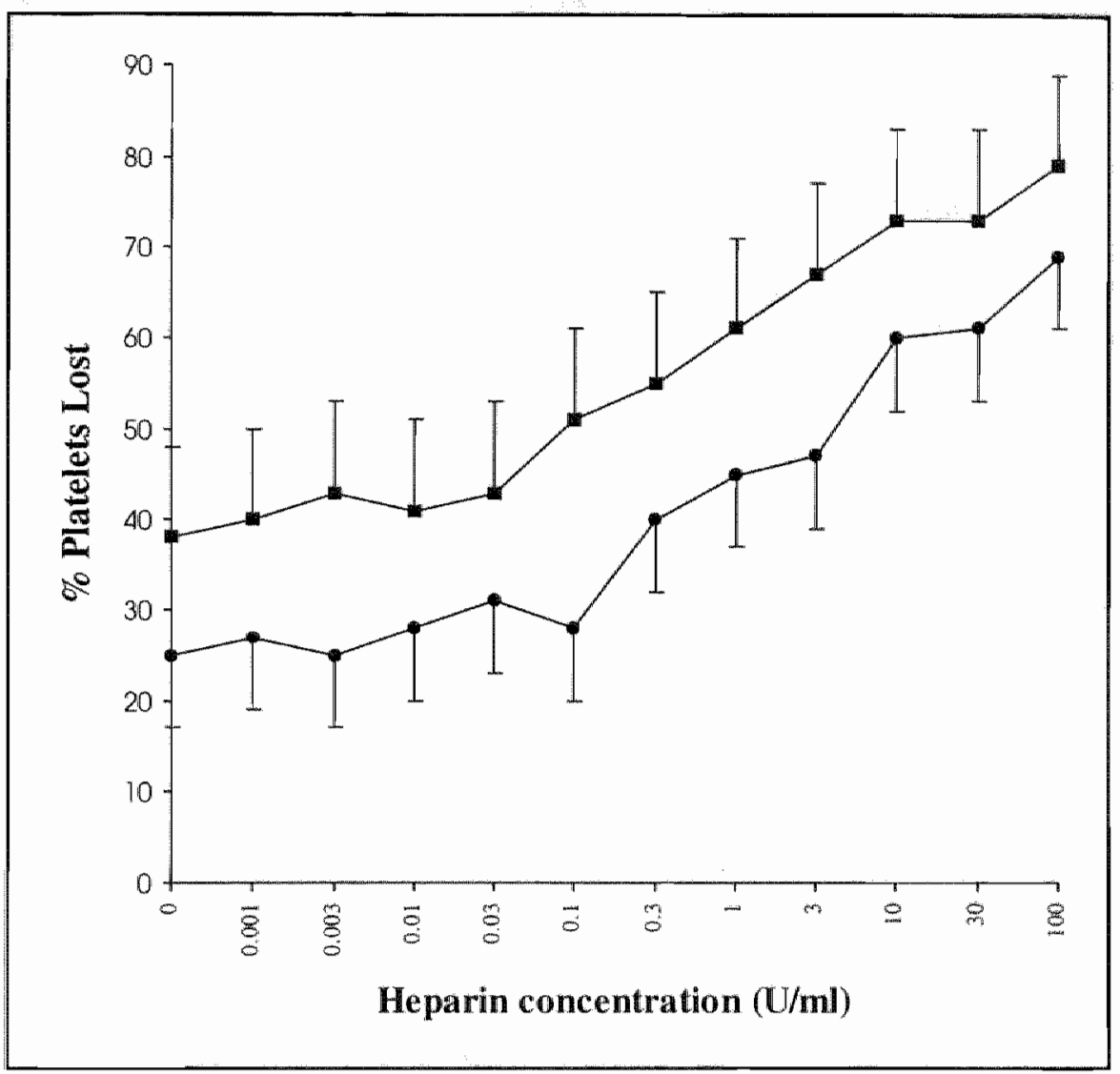

Figure 5

Fig. 5

The results shown are the mean $\%$ platelet loss plus the SEM. The mean HIA threshold measurements in heparinised blood from pregnant subjects ( $\mathrm{n}=5$ ) before aspirin ingestion ( $)$, and 1 hour post ingestion (-). 


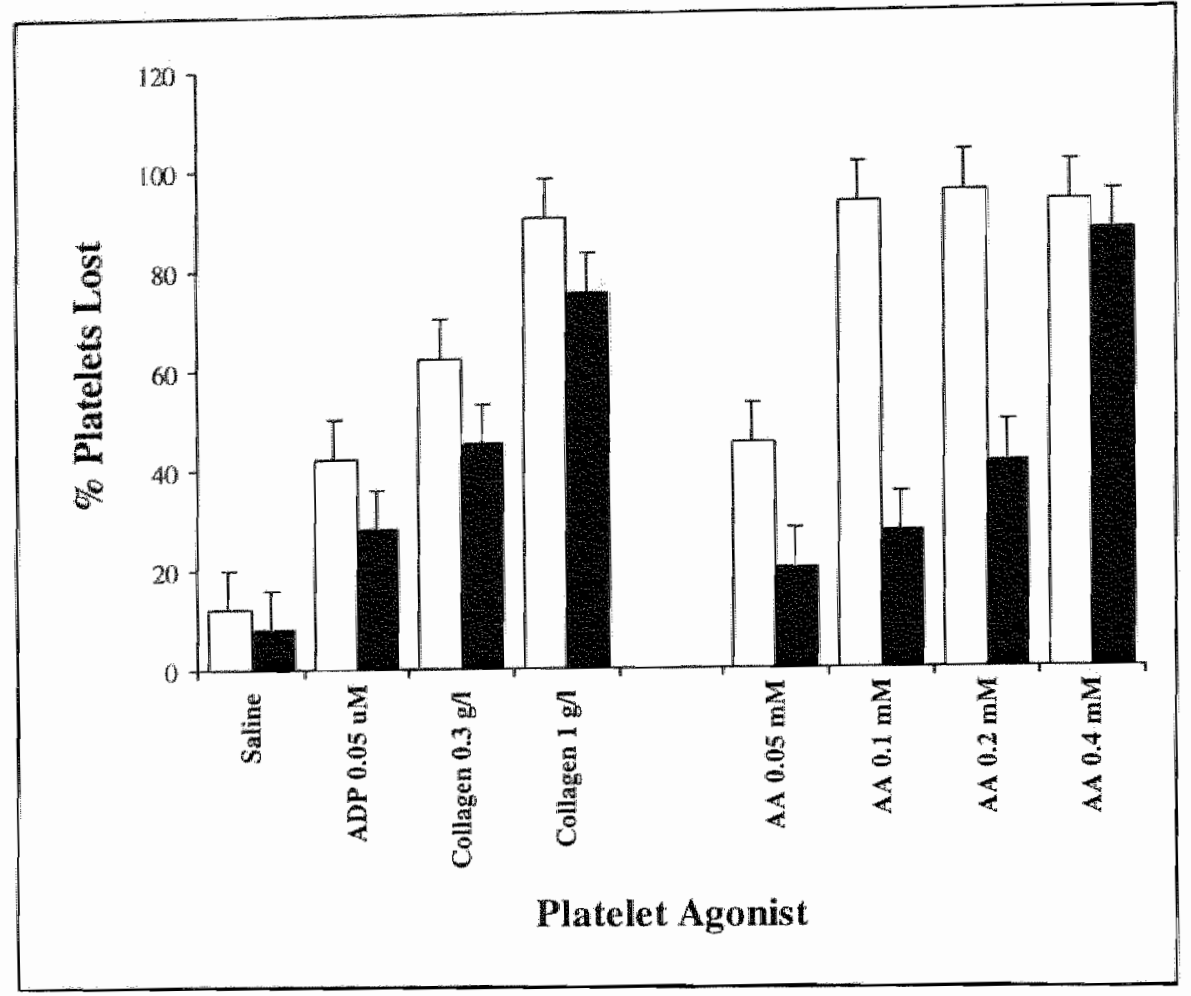

\section{Figure 6}

Fig. 6

The results shown are the mean o platelet loss plus the SEM. Citrated blood from 10 pregnant subjects taken just before (open bars); and 1 hour after (solid bars); the ingestion of $75 \mathrm{mg}$ aspirin. The concentrations of platelet agonists used are described in Table 5.

\section{Discussion}

Further aspects of the mechanism of heparin-induced platelet aggregation (HIA) have been investigated in this study. The results provide evidence that an, as yet, undefined 'factor' present in plasma is responsible for the increased extent of heparin-induced aggregation (HIA) seen in pregnant females. HIA could be stimulated in a whole blood sample from one subject by adding the plasma from another subject. Heating the plasma for 20 minutes at $56^{\circ} \mathrm{C}$ did not change the extent of HIA induced, suggesting that the "HIA factor" is a heat stable, plasma component. Johnson et al (1984) reported the use of heat-treated serum in their test for HA supporting the idea that this plasma factor is heat stable. Certain plasma induced very rapid ( $<30$ seconds) HIA when added to whole blood, 
whereas other plasma needed to be incubated for several minutes with the blood before the HIA became maximal.

HIA could be induced in the whole blood from pregnant subjects at concentrations of heparin as low as $0.1 \mathrm{U} / \mathrm{ml}$, and the effect could also be demonstrated, to a lesser extent, in blood collected from non-pregnant women. This led to attempt to use the whole aggregation technique to quantify HIA. The method involved exposing citrated blood to a range of heparin concentrations and then measuring the extent of platelet loss on stirring. A "HIA Threshold" was defined as a $10 \%$ increase in aggregation (\% platelets lost) due to heparin addition. These "HIA Threshold" measurements were made in four patient groups; pregnant and non-pregnant females, normo- and hypertensive males. When the full threshold testing profiles were compared, the subject groups were clearly quite different (Figure 2). The highest heparin-induced platelet loss (HIA) occurred in the normotensive pregnancy group, and progressively less HIA was seen in the hypertensive males, the non-pregnant females, with the normotensive males showing the least HIA. The extent of 'spontaneous platelet aggregation' (SPA) in these groups also varied (Figure 2), being higher in pregnant females and hypertensive males $(p<0.05)$. If the SPA is subtracted from the HIA results obtained with both the normotensive and hypertensive males, then the response of both groups to heparin is similar. Further hypertensive males should be tested as conclusions can only be limited with such a small study group $(n=3)$. When the "HIA Thresholds" for the individual subjects are calculated (Table 3 ) there is significant of overlap between the subject groups. However, the pregnant women clearly have a greater proportion of low thresholds, which agrees with our initial report (Burgess-Wilson et al 1986). There are some low threshold values found in non-pregnant women. This group is worth further study to determine if they are at risk of developing HIT. The males on average had higher thresholds than females, with hypertensive men being similar to normotensive men as previously mentioned. The only significant differences seen between the groups in the other parameters measured are that the pregnant subjects are younger, and had lower red cell counts than the other three groups. The observed reduction of the red cell count is a normal feature of pregnancy, and it is difficult to link this observation with the low HIA thresholds, because the pattern of increase in HIA in pregnancy does not correlate with the changes in red cell count (Burgess-Wilson et al 1986).

If we conclude that some plasma factor that causes HIA, and that this protein/antibody is present at increased levels during pregnancy, then it appears that this substance also exists in non-pregnant females at lower levels. In our studies it was possible to stimulate HIA (using the HIA threshold as defined) in $82 \%$ of the pregnant subjects, and $44 \%$ of the non pregnant females with $0.3 \mathrm{U} / \mathrm{m}$ 
of heparin (a level that are commonly achieved during its therapeutic use). It is likely that even higher levels are achieved during heparin therapy at some points, and that HIA might be therefore be stimulated in large percentage of females. However, although it is possible to create this effect in vitro, the incidence of HIT with clinical complications is quite low. It has been reported that many patients on heparin do develop, transiently, a mild thrombocytopenia (Johnson et al 1984), and it is possible that the HIA Threshold measurement might be able to predict these patients. Perhaps the definition of the 'HIA threshold' will have to be changed to allow identification of patients where severe, persistent thrombocytopenia could occur. It is entirely possible, however, that the artificial environment of blood in the test procedure allows HIA to occur in witro, when it would not occur in vivo, and only a clinical study of patients receiving heparin will answer this question. The relevance of any in vitro test should be questioned until the appropriate clinical studies have been performed.

Anti-platelet agents have been used in many conditions where it is felt that increased platelet activity might lead to thrombosis. In the case of heparininduced thrombocytopenia (HIT), bleeding rather than thrombosis is a more usual complication, although thrombosis does occasionally occur. In pregnancy there is evidence that platelets have increased activity (Lewis et al 1980, Andrews et al 1985, Hoche et al 1983, Briel and Lippert 1983). Anti-platelet treatment in the form of dipyridamole and aspirin, was given to a group of mothers who had a high risk of developing pregnancy-induced hypertension, and it significantly improved foetal outcome (Beaufils et al 1985). Our in vitro studies of anti-platelet agents gave clear indications that both SPA and HIA could be inhibited by aspirin, prostacyclin, apyrase and dipyridamole. The concentrations used, however, were somewhat higher than those achieved in vivo during therapy. In our experiments we normally stimulated HIA with $10 \mathrm{U} / \mathrm{ml}$ heparin, but if $1 \mathrm{U} / \mathrm{ml}$ heparin was used a greater inhibition of HIA occurred (Figure 4). Further in vitro studies showed that dipyridamole at concentrations of $10-30 \mu \mathrm{M}$ significantly $(\mathrm{p}<0.01)$ reduced the extent of HIA, and that aspirin $(100 \mu \mathrm{M})$ also showed inhibition of SPA in whole blood, but not HIA. An ex-vivo study of aspirin ingestion $(75 \mathrm{mg}$ ) during late pregnancy also showed evidence of a reduction in platelet activity. There was no significant effect on the HIA threshold which increased post aspirin to $1.88 \mathrm{U} / \mathrm{ml}$, compared with $0.76 \mathrm{U} / \mathrm{ml}$ pre ingestion. Agonist-induced aggregation results showed that aspirin significantly reduced AA-induced aggregation (at concentrations of 0.1 and $0.2 \mathrm{mM}$ ). A reduction in the aggregation induced by ADP and collagen and SPA was seen but was not significant. The SPA results were obtained on blood tested 10 minutes after venepuncture. When blood was tested 30 minutes after venepuncture the extent of SPA was greater and there was a significant reduction $(\mathrm{p}<0.05)$ in SPA due to 
aspirin. Louden et al (1992) in a more comprehensive study has also looked at the effect of aspirin (60mg daily) on SPA and found no effect. It is, however, unclear exactly how long after venepuncture the blood was tested. This emphasises the effect that different methodology can have on the results obtained and the conclusions drawn.

\section{Conclusions}

In this study heparin-induced aggregation (HIA) appeared to be due to a heat stable "factor" present in plasma. This supports the findings of Salem and Van der Weyden (1983), Sheridan et al (1986), Saba et al (1984) and Blockmans et al (1986). The study of normal males and females suggested that this "HIA factor" might be present in the general population, but is at a higher concentration in females than in males. Previous studies (Burgess-Wilson et al 1986) suggested that the "HIA factor" reached very high levels during late pregnancy. Conversely, the increases in HIA seen might be due to a reduction in the level of some other substance that normally inhibits HIA. It would be useful to isolate and identify the "factor", or the lack of it. This might lead to a better understanding of whole blood aggregation in general, the relevance of the measurement of platelet aggregation in whole blood, its relationship to the activation status of platelets and to the clinical condition of heparin-induced thrombocytopenia (HIT).

The in vitro and ex vivo studies suggest that certain anti-platelet agents, namely apyrase $(1 \mathrm{U} / \mathrm{ml})$, prostacyclin $(0.5 \mathrm{ng} / \mathrm{ml})$ and dipyridamole $(10 \mu \mathrm{M})$ inhibit HIA. As these all have rather different modes of action, it is not possible to conclude the mechanism of the inhibition. Aspirin ( $10-4 \mathrm{M}$ ) and a thromboxane synthetase inhibitor UK $37248(10.4 \mathrm{M})$ had no significant effect in vitro. In an ex vivo study, aspirin at a concentration that reduced AA-induced aggregation did not inhibit HIA, but appeared to inhibit SPA.

Using whole blood platelet aggregation it is possible to quantify the platelets' sensitively to heparin (i.e. measure the "HIA threshold"). Whole blood platelet aggregation techniques do not appear to been used previously in any prospective studies of patients receiving heparin. Such a study, measuring the "HIA threshold', would allow the method to be evaluated as a tool for detecting patients at risk of developing heparin-induced thrombocytopenia (HIT). At present there seems to be no single, universally accepted test of choice for the detection of HIT (Kelton 1994, Messmore et al 1994, Greinacher et al 1994) and an adaptation of the HIA threshold measurement described here might perhaps provides a more simple, relevant test for the clinician. 


\section{Acknowledgments}

I would like to thank the Prof. Symonds and the Department of Obstetrics and Gynaecology, Queens Medical Centre, University Hospital Nottingham for kind permission to take blood samples from their patients and for supporting the ex vivo aspirin study. The Haematology Department, University Hospital Nottingham for performing the full blood counts.

\section{References}

Andrews NP, Broughton Pipkin F and Heptinstall S. Blood platelet behaviour in mothers and neonates. Thromb Haemost 1985; 53: 428-432.

Beaufils M, Uzan S, Donsimoni R and Colan JC. Prevention of pre-eclampsia by early antiplatelet therapy. Lancer April 13 1985; i: 840-842.

Bevan J and Heptinstall S. Serotonin-induced platelet aggregation in whole blood and the effects of ketanserin and mepyramine. Thromb Res 1985; 38: 189-194.

Blockmans D, Bounameatux $H$, Vermylen $J$ and Verstraete $M$. Heparin-induced thrombocytopenia. Platelet aggregation studies in the presence of heparin fractions or semisynthetic analogues of various molecular weights and anticoagulant activities. Thromb Haemost 1986; 55(1): 90-93.

Briel RC and Lippert TH. Platelet sensitivity to prostacyelin in normal and complicated pregnancy. In: Prostacyclin in pregnancy, Lewis PJ, Ed. Raven Press, New York, 1983, pp 195-197.

Burgess-Wilson ME, Morrison R and Heptinstall S. Spontaneous platelet aggregation in heparinised blood during pregnancy. Thromb Res 1986; 37: 385-393.

Greinacher A, Michels I, Kiefel $V$ and Mueller-Eckhardt $C$. A rapid and sensitive test for diagnosing heparin-associated thrombocytopenia. Thromb Haemost 1991; 66: 734-736

Greinacher A, Amiral J, Dummel V, Vissac A, Kiefel V and Mueller-Eckhardt C. Laboratory diagnosis of heparin-induced thrombocytopenia and comparison of platelet aggregation test, heparin-induced platelet activation test, and platelet factor theparin enzyme-linked immunosorbant assay. Transfusion 1994; 34: $381-385$.

Hoche C, Kefalides A, Dadad C and Sinzinger H. Platelet sensitivity to prostacyclin in pregnancy and puerperium. Im: Prostacyclin in pregnancy, Lewis PJ, Ed. Raven Press, New York, 1983, pp 189-193.

Johnson RA, Lazarus $\mathrm{KH}$ and Henry DH. Heparin-induced thrombocytopenia: a prospective study. Am J Hematol 1984: 17: 349-353. 
Kelton JG, Sheridan D, Brain H, Powers PJ, Turpie AG and Carter CJ. Clinical usefulness of testing for a heparin-dependent platelet-aggregating factor in patients with suspected heparinassociated thrombocytopenia. J Lab Clin Med 1984; 103(4): 606-612.

Kelton JG. Heparin induced thrombocytopenia. Hemostasis and thrombosis update 94 . II th Annual 3 Day Seminary and Workshop. Aprill 1994, pp $15-19$.

Lewis PJ, Boylan P, Friedman LA, Hensby CN and Downing 1. Prostacyclin in pregnancy. BMJ 1980;280: $1581-1582$.

Louden KA, Broughton Pipkin F, Symonds EM, Tuohy P, O'Callaghan C, Heptinstall S, Fox S and Mitchell JRA. A randomized placebo-controlled study of the effect of low dose aspirin on platelet reactivity and serum thromboxane $B_{2}$ production in non-pregnant women, in normal pregnancy, and in gestational hypertension. Br J Obstet Gynaccol 1992; 99: 371-376.

Messmore H, Koza M, Griffin N, Farid $S$ and Fabbrimi N. The specificity of tests for heparin induced thrombocytopenia of the immune type. Frontiers in vascular medicine. An international symposium, Frankfurt 1994. Abstract, pp 37.

Saba HI, Saba SR and Morelli GA. Effect of heparin on platelet aggregation. Am J Hematol $1984 ; 17: 295-306$.

Salem HH and Van der Weyden MB. Heparin-induced thrombocytopenia. Variable plateletrich plasma reactivity to heparin dependent platelet aggregating factor. Pathology 1983;15: 297-299.

Sheridan D, Carter C and Kelton JG. A diagnostic test for heparin-induced thrombocytopeniat. Blood 1986; 67(1): 27-30. 


\title{
CHAPTER 5
}

\section{Spontaneous Platelet Aggregation in Whole Blood from Normal Individuals}

\begin{abstract}
Two studies of spontaneous whole blood platelet aggregation were performed. The first was a preliminary study that investigated 67 nomal healthy individuals and showed strong positive correlation's between the extent of whole blood aggregation and the age and haematocrit of the individual. However, because of the small data base and the age/sex distribution of the group, it was not possible to exclude the possibility that correlation"s of whole blood aggregation with other parameters had arisen because of their association with age. In the second study 102 nomal healthy individuals were investigated. The strong association between the extent of whole blood aggregation with age was again seen, but all other correlation"s where shown to be indirect.
\end{abstract}

5.1 Spontaneous Platelet Aggregation in Whole Blood: Dependence on age and haematocrit.

5.2 Spontaneous whole blood platelet aggregation in 102 normal individuals: A strong association with age. 


\subsection{Spontaneous Platelet Aggregation in Whole Blood : Dependence on age and haematocrit.}

Burgess-Wilson ME, Green S, Heptinstall S and Mitchell J.R.A. Letter. Lancet, Nov 24, 1984; ii: pp 1213.

\section{Abstract}

Blood samples were taken from 67 normal individuals and were tested using the Ultra-Flo 100 to measure whole blood aggregation. Several other parameters that have been used to assess the risk of thrombosis were also measured, and compared with the extent of whole blood aggregation measured. Positive correlation between 'Spontaneous" platelet aggregation (SPA) and age, fibrinogen level, haematocrit and platelet count were observed. Regression analysis revealed that the major correlation's were between SPA in citrated blood and age $(p<0.01)$ and haematocrit $(p<0.01)$. This suggests that SPA may be a useful risk marker for thrombosis. 
Sir,- Two groups have reported on the effect of haematocrit (Hot) on spontaneous platelet aggregation (SPA) in whole blood. Saniabadi et al (1) and now Dr Harrison and his colleagues (Oct 27, p.991) manipulated Hot in vitro and showed that SPA, as measured by the fall in number of single platelets in stirred citrated blood, increases with increasing Hct. We have measured SPA in citrated blood and in heparinised blood obtained from different subjects and compared the values obtained with the Hct of the samples. We also looked for relationship between SPA and age, plasma fibrinogen (a co-factor in the aggregation process), and several other haematological indices and found that SPA correlates with age, Hct, fibrinogen, and platelet count, the major independent determinants being age and Hot.

Blood was taken from 67 healthy subjects aged 18-88 years, and collected into citrate, heparin and EDTA (2). Citrated and heparinised samples were used to determine SPA; $400 \mu \mathrm{l}$ volumes were stirred at $37^{\circ} \mathrm{C}$ for $8 \mathrm{~min}$. and the number of single platelets that remained were determined in an 'Ultra Flo $100^{\prime}$ wholeblood platelet counter (2). The fall in the number of single platelets was expressed as a percentage of the number of platelets in the EDTA blood. Plasma fibrinogen was determined (3) in 31 of the citrated samples, and Hct and the other haematological indices were measured in 54 of the EDTA samples.

SPA in whole blood taken from different individuals ranged from 0 to $96 \%$ (citrated) and 8 to $82 \%$ (heparinised). After the samples had been stirred for 8 minutes the values obtained in citrated blood and heparinised blood for the different individuals were closely correlated $(r=0.87, p<0.001)$, so SPA does not vary greatly with the type of anticoagulant. Significant positive correlation's were found between SPA (citrated blood) and the individual's age, fibrinogen, haematocrit and other red-cell related indices, and platelet count (Table 1). Multiple regression analysis showed that the major contributors to the variation in SPA in citrated blood are age $(p<0.01)$ and Hct $(p<0.01)$, the contributions from fibrinogen and platelet count being non-significant. 
Table 1 Correlation's between SPA in citrated blood and other indices

\begin{tabular}{|l|c|c|}
\hline & $\boldsymbol{r}$ & $\mathrm{p}$ \\
\hline Age & 0.57 & $<0.001$ \\
\hline Haematocrit & 0.46 & $<0.001$ \\
\hline Fibrinogen & 0.59 & $<0.001$ \\
\hline Haemoglobin & 0.43 & $<0.01$ \\
\hline Red cell volume & 0.33 & $<0.02$ \\
\hline Platelet count & 0.31 & $<0.02$ \\
\hline
\end{tabular}

The clear relation between SPA and Hct that we and others have demonstrated may be a consequence of ADP being liberated from red cells. In 1960, Hellem (4) showed that the number of platelets that are lost when whole blood is passed through a column of glass beads is proportional to the Het of the sample, and this relation was attributed to ADP liberated from the red cells (5). Using an enzyme system that degrades ADP, Harrison and Mitchell (6) provided evidence that ADP liberated from red cells is a major contributor to the loss of single platelets from blood held in a rotating glass flask. Both Fox et al (2) and Saniabadi et all (7) have shown that enzymes that degrade ADP reduce SPA in stirred whole blood, and Harrison and colleagues have now shown that increased SPA at high Hct is associated with increased red cell damage.

SPA may be merely an in-vitro artifact of red cell damage and thus irrelevant to the in-vivo situation. However, the number of single platelets in whole blood falls even when the blood is simply allowed to stand on the bench (2) and it is possible that red cells are continually liberating small quantities of ADP and that this happens in vivo as well as in vitro. This could explain why there is an increased risk of thrombosis when Hct is high (8). Our results also show a clear relation between SPA in whole-blood and the subjects' age that is independent of Het, and there is no doubt that the risk of an individual experiencing coronary heart disease, stroke, or peripheral arterial disease increases with age (9). SPA may itself be a risk marker for thrombosis. Large-scale prospective studies in which this rapid and simple test of platelet behaviour is measured are now required to test this hypothesis. 
We thank Miss J.Crawford and Miss M.Hawkins for technical assistance and staff in the Haematology department, University Hospital, Nottingham, for the full blood counts. The work was supported by CORDA.

\section{References}

1. Saniabadi AR, Lowe GDO, Barbenel JC and Forbes CD. Haematocrit, bleeding time. and platelet aggregation. Lancet 1984; i: pp 1409-1410.

2. Fox SC, Burgess-Wilson M, Heptinstall $S$ and Mitchell JRA. Platelet aggregation in whole blood determined using the Ultra-Flo 100 platelet counter. Thromb Haemost 1982; 48: $327-329$.

3. Clauss Von A. Gerinnungsphysiologische schellmethode zur bestimmung des fibrinogens. Acta Haematol 1957; 17:237-247.

4. Hellem AJ. The adhesiveness of human blood platelets in vitro. Scand I Clin Lab Invest 1960: 12 (suppl 51).

5. Gaarder A, Jonsen J, Laland S, Hellem A and Owren PA. Adenosine diphosphate in red cells as a factor in the adhesiveness of human blood platelets. Nature 1961; 192: 531 532.

6. Harrison MJG and Mitchell JRA. The influence of red blood-cells on platelet adhesiveness. Lancet 1966; ii: pp 1163-64.

7. Saniabadi AR, Lowe GDO, Barbenel JC, Forbes CD. A comparison of spontaneous platelet aggregation in whole blood with platelet rich plasma: additional evidence for the role of ADP. Thromb Haemost 1984; 51: 115-18.

8. Dormandy JA. Haemorheology and thrombosis. In : Haemostasis and thrombosis. Bloom AL, Thomas DP, eds. Churchill Livingstone, Edinburgh, 1981, pp 610-625.

9. Dawber TR. Incidence of coronary heart disease, stroke, and peripheral arterial discase. In: The Framingham Study. Harvard University Press, Cambridge, Mass, 1980, pp 59. 75. 


\title{
5.2 Spontaneous whole blood platelet aggregation in 102 normal individuals : A strong association with age
}

Burgess-Wilson ME, Green S, Heptinstall S and Mitchell JRA. Submitted to Haemostasis (in press).

\begin{abstract}
A study of 102 normal healthy individuals showed a strong association of whole blood aggregation with age. This contrasted with a previous study of normal individuals where a correlation was also found between whole blood aggregation and haematocrit. The age/sex distribution of the individuals in this second study was more evenly spread, and all parameters where measured on each subject. When this larger population was tested all other correlation's where shown to be indirect. The previous correlation seen with haematocrit appears to be due to the composition of the group used. This stresses the importance of using large, well defined groups of subjects, with a careful examination of the parameter distributions.
\end{abstract}




\section{Introduction}

Although the concept that platelet hyperaggregability may contribute to thrombus formation is an attractive one, measurements of platelet aggregation in vitro have not yet proved to be of any predictive or diagnostic value in thrombotic disease. Platelet aggregation is usually studied after the platelets have been separated from other blood cells, by the preparation of platelet-rich plasma (PRP). This removes them from the physical influence of the other cells present in circulating blood, and from the influence of substances released from these cells. It is, however, possible to measure platelet aggregation in whole blood using a particle counting technique (Butchers et al 1980, Nunn and White 1980, Lumley and Humphrey 1981, Fox et al 1982). This involves counting the number of single platelets that remain in an anticoagulated whole blood sample as aggregation proceeds.

In an evaluation of this method it was observed that aggregation occurred when blood was stirred without that addition of agonists (Fox et al 1982). This phenomenon was called "spontaneous" platelet aggregation (SPA), whilst acknowledging that it was really stirring incluced aggregation, possibly due to ADP released from red cells that are damaged by the stirring. The extent of SPA was relatively similar for any normal subject studied over a period of months. The extent of SPA found in different normal subjects varied considerably, and an attempt was made to establish a normal range of results for SPA in citrated and heparinised blood.

However, when the results from a group of 67 normal individuals were analysed, the extent of SPA correlated strongly with the subjects age (BurgessWilson et al 1984). The extent of SPA also correlated with the individuals, haematocrit (Hct), fibrinogen level, haemoglobin value, red cell count and platelet count. But the main correlation's were between SPA and age, and between SPA and Hct. The results appeared to confirm the findings of other groups that found a correlation between SPA and Hct (Saniabadi et al 1984, Harrison et al 1984, Lowe and Forbes 1985). This relation between SPA and Hot was thought to be a consequence of ADP being liberated from red cells. The leakage of ADP from red cells and its effect on platelets had been previously described by several other investigators (Hellem 1960, Gaarder et al 1961, Harrison and Mitchell 1966, Saniabadi et al 1984).

Some studies have shown Hct, fibrinogen, viscosity and platelet count to be positively associated with increased risk of thromboembolic events such as coronary heart disease, myocardial infarction, stroke and peripheral arterial 
disease (Fulton and Duckett 1976, Preston et al 1979. Dawber 1980, Lowe et al 1980, Meade et al 1980, Dormandy 1981. Haines et al 1983).

It could not exclude the possibility that some of the correlation"s reported were indirect and due to associations between other parameters. Therefore testing continued until a much larger and complete database (comprising of complete sets of data from 102 normal individuals) was available. The statistical analysis of this data is presented in this report.

\section{Reagents and Methods}

Blood collection and testing. Blood was taken into citrate, heparin and EDTA and tested as previously described (Burgess-Wilson et al 1986) using the method described by Fox et al 1982. The other parameters (Table 1), where tested as previously described (Burgess-Wilson et al 1986), and the normal ranges are described by Eastham (1974).

Subjects tested. Blood was obtained from 102 different individuals, 50 males and 52 females with ages ranging from 18-88 years. Blood was collected from volunteers in the Department of Medicine, the coronary care unit as visitors, endoscopy day-patients (with no disease), the hospital cleaning staff and eye clinic out-patients. In the older subjects it is not possible to exclude all illness or some form of medication. But in all cases subjects with known bleeding or clotting disorders were excluded, and those subjects on any medications known to alter platelet behaviour were also excluded. Testing was performed over a twoyear period.

\section{Results}

The mean results obtained for the males and females is shown in Table 2. As expected the haemoglobin, Hct and red cell count were lower in females. All other parameters tested showed no significant difference due to gender. In this analysis the data from males and females were combined because there was no evidence that the relationships studied differed in males and females. 
Table 1 Normal ranges

\begin{tabular}{|l|c|c|c|c|}
\hline \multirow{2}{*}{ Parameter } & Units & \multicolumn{2}{|c|}{ Normal ranges } & $\begin{array}{c}\text { Abbreviation } \\
\text { used }\end{array}$ \\
\cline { 3 - 5 } & Years & Males & Females & \\
\hline Age & $\mathrm{mg} / \mathrm{dl}$ & $200-400$ & $200-400$ & Fib \\
\hline Fibrinogen & $\times 10^{9} / 1$ & $4-10$ & $4-10$ & WCC \\
\hline White cell count & $\times 10^{12} / 1$ & $4.5-6.5$ & $3.9-5.6$ & RBC \\
\hline Red cell count & $\times 10^{9} / 1$ & $150-340$ & $150-340$ & PBC \\
\hline Platelet count & 19 & $84-99$ & $84-99$ & MCV \\
\hline Mean (red) cell volume & $\mathrm{mg} / \mathrm{dl}$ & $13.5-18.0$ & $11.5-16.4$ & $\mathrm{Hb}$ \\
\hline Haemoglobin & - & $40-54$ & $36-47$ & $\mathrm{Hct}$ \\
\hline Haematocrit & $\mathrm{pg}$ & $27-32$ & $27-32$ & MCH \\
\hline Mean red cell Hb & $\%$ & Unknown & Unknown & SPA (Citrate) \\
\hline SPA (in citrate) & $\%$ & Unknown & Unknown & SPA (Heparin) \\
\hline SPA (in heparin) & & &
\end{tabular}

The strong association of the extent of SPA (after 8 minutes stirring) with age was found as described previously (Burgess-Wilson et al 1984). The individual SPA results can be seen in Figure 1 plotted against age. The SPA results obtained in citrated and heparinised blood were quite similar (Figure 2). There were some differences in the relationships between the two types of blood, but the differences were not statistically significant. More data would be needed for a rigorous comparison of the relationships for the two types of blood.

Table 3 shows the correlation coefficients (r) found. Although the three parameters; fibrinogen, WCC and MCV were positively associated with age, when the data was adjusted for age the WCC and MCV no longer showed statistically significant correlation with SPA. The fibrinogen did remain significantlly correlated with SPA after age adjustment. Figure 3 shows the individual fibrinogen levels plotted against the SPA seen in citrated blood. If, however, the two male subjects that had abnormally high fibrinogen levels ( 4.8 and $5.7 \mathrm{mg} / \mathrm{dl}$ ), are excluded from the analysis, the correlation between SPA and fibrinogen disappears. Further analysis showed that there is little relationship between fibrinogen and $\mathrm{WCC}$ and no relationship between fibrinogen and $\mathrm{MCV}$. 
Table 2 Mean values found in males and females

\begin{tabular}{|l|c|c|c|c|}
\hline Parameter & \multicolumn{2}{|c|}{ Males $(\mathbf{n = 5 0 )}$} & \multicolumn{2}{c|}{ Females $(\mathbf{n}=\mathbf{5 2})$} \\
\hline & Mean & SD & Mean & SD \\
\hline Age & 50.7 & 20.3 & 47 & 18.3 \\
\hline Fibrinogen & 2.66 & 0.8 & 2.64 & 0.49 \\
\hline Platelet count (PBC) & 282 & 63 & 289 & 66 \\
\hline WCC & 6.9 & 1.6 & 6.4 & 1.7 \\
\hline RBC & 4.9 & 0.5 & 4.4 & 0.4 \\
\hline MCV & 91 & 5 & 90 & 4.5 \\
\hline Hb & 14.8 & 1.1 & 13.2 & 1.2 \\
\hline Hct & 0.45 & 0.04 & 0.40 & 0.04 \\
\hline MCH & 30.0 & 1.9 & 29.9 & 1.8 \\
\hline SPA (Citrate) & 41.0 & 22.8 & 38.2 & 21.3 \\
\hline SPA (Heparin) & 43.9 & 16.1 & 42.9 & 14.6 \\
\hline
\end{tabular}

Note. The units and normal ranges can be found in Table 1.

Table 3 Correlation's with SPA and age

\begin{tabular}{|l|l|l|}
\hline \multirow{2}{*}{ Parameter } & \multicolumn{2}{|c|}{ rvalues } \\
\cline { 2 - 3 } & SPA & Age \\
\hline Age & 0.58 & - \\
\hline Fibrinogen & 0.45 & 0.51 \\
\hline WCC & 0.26 & 0.24 \\
\hline MCV & 0.23 & 0.18 \\
\hline
\end{tabular}




\section{Discussion and conclusions}

In this study of SPA in citrated and heparinised blood from normal subjects, a strong correlation between SPA and the subjects age was found, supporting our previous report (Burgess-Wilson et al 1984). After stirring for 6-8 minutes there was no difference in the number of platelets lost in citrated blood (CB) compared with heparinised blood (HB). However, before stirring started and after stirring for only 2 or 4 minutes the number of platelets lost in $\mathrm{HB}$ was significantly higher than in $\mathrm{CB}$. The experimental procedure was such that the $\mathrm{CB}$ was always studied exactly 30 minutes after venepuncture and the $\mathrm{HB}$ exactly 40 minutes after venepuncture. Although, as described previously (Fox et al 1982), the extent of SPA increases when the blood is left standing this time difference was sufficient to account for our observation. The reduced number of platelets in the heparinised blood is due to the heparin.

Statistical analysis in this study showed that, with the exception of fibrinogen, all the other associations with SPA, which had been previously seen, could be removed by adjusting the analysis to account for the subjects age.

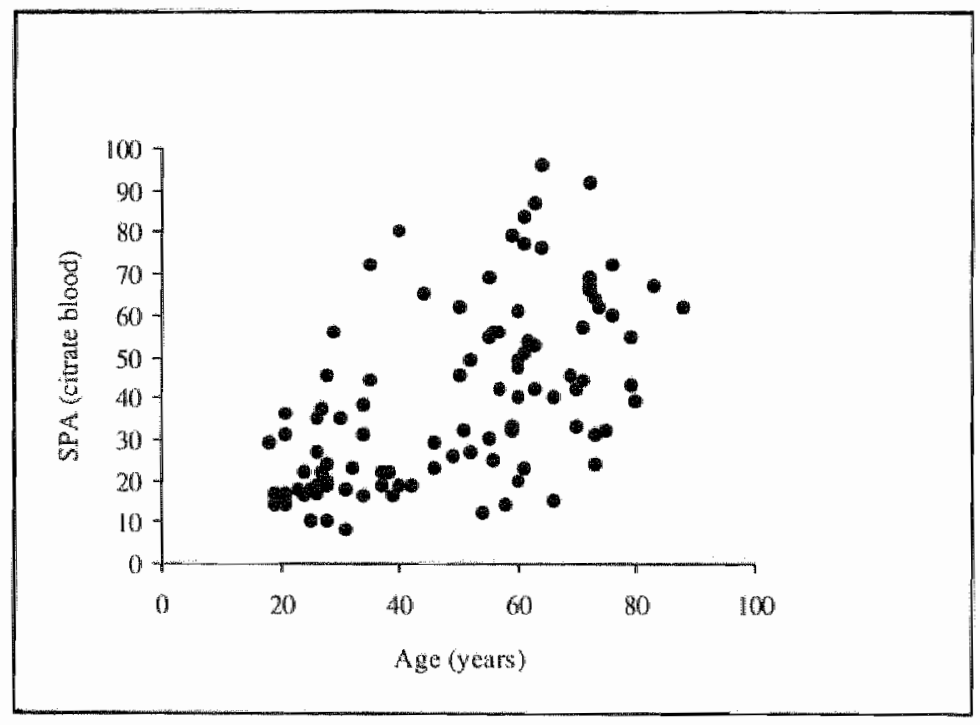

\section{Figure 1}

Fig. I The association between SPA (\% platelets lost after 8 minutes stiring) in citrated blood and the subjects age in years. 


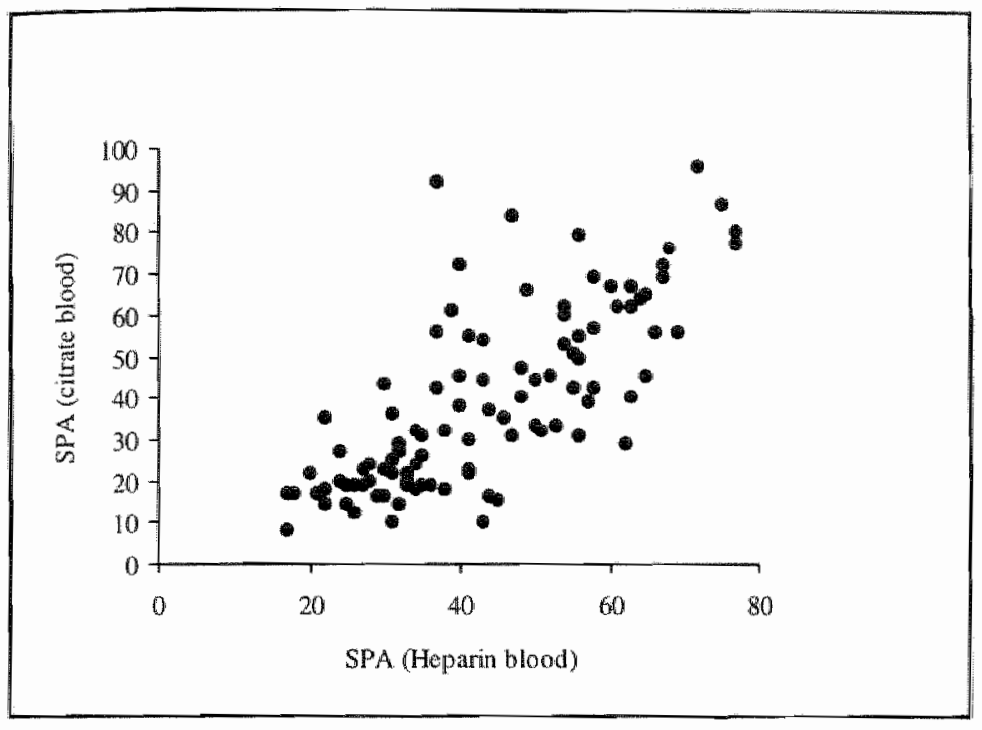

Figure 2

Fig. 2 The association between SPA in citrated blood (\% platelets lost after 8 minutes stirring) and SPA in heparinised blood (\% platelets lost after 8 minutes stiming).

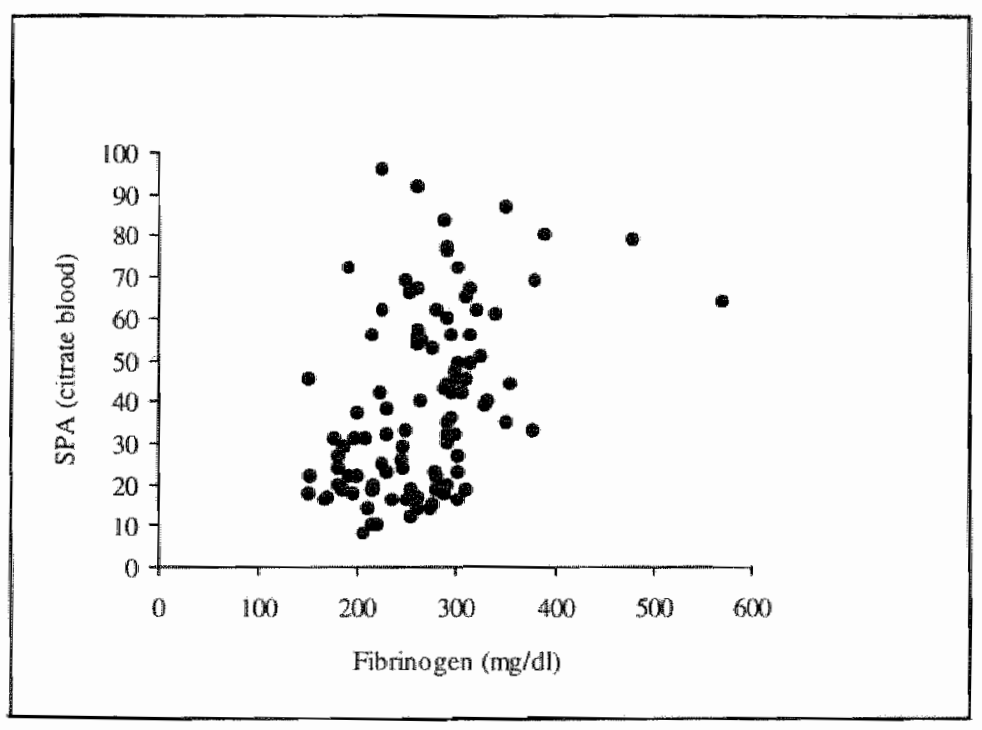

\section{Figure 3}

Fig. 3 The association between SPA in citrated blood (\% platelets lost after 8 minules stirring) and fibrinogen ( $\mathrm{mg} / \mathrm{dl}$ ). 
The relationship between fibrinogen and SPA (see Figure 3) was, however, dependent on two results (older males with abnormally high fibrinogen levels; 4.8 and $5.7 \mathrm{mg} / \mathrm{dl}$ ). When these results were excluded from the analysis statistical evidence for a relationship between SPA and fibrinogen disappeared.

It was surprising that the previously strong association of SPA and Het had disappeared because other investigators had also shown a correlation between SPA and Hct (Saniabadi et al 1984, Harrison et al 1984, Lowe and Forbes 1985)), and demonstrated that a high Hct can lead to an increased risk of thrombosis (Dormandy 1981). The original data group of 67 normals was reviewed and it was discovered that there was a high proportion of both young females and old males in this group. This imbalance had created a correlation between those parameters that are lower in females (Hct, haemoglobin and red cell count), with the subjects age. Only with a larger, more complete group where the age range was covered more evenly in both the male and females groups, did this artifactual relationship disappear. From this experience it is advised that when groups of "normal" or abnormal subjects are being investigated and a relationship with age is evident, care should be taken to ensure that the whole age range is represented evenly by the group.

There is no doubt that the risk of an individual experiencing coronary heart disease, stroke of peripheral arterial disease increases with age (Dawber 1980). There is also evidence that Hct and fibrinogen are risk-markers for thrombosis (Fulton and Duckett 1976, Lowe et al 1980, Meade et al 1980, Haines et al 1983). Indeed, fibrinogen and Hct are major determinants of blood viscosity, and the contribution of blood viscosity to thrombosis has been reviewed by Dormandy (1.981). So, with the results of this study, it is difficult to explain the relationship of SPA to age with our current understanding of platelet behaviour. There appears to be no obvious relationship in this group of normal individuals of SPA with the red cells or the fibrinogen level, both of which might be expected to effect platelet behaviour. It is perhaps possible that there are small age-related changes in these, and other parameters, that may act synergistically to create an increase in platelet reactivity with age, which we are able to measure using a whole blood aggregation technique. It is also possible that the increased SPA we observed does not predict thrombosis risk in normal subjects, and the relationship with age is due to some other effect of age.

Large scale prospective studies, in which SPA is measured in a much larger group of normal subjects will need to be made before the real diagnostic use of SPA is known. These results do suggest though, that any studies of whole blood 
aggregation where normal subjects are compared to abnormal subjects, the normal controls should be carefully age-matched.

\section{Acknowledgments}

We are very grateful for the statistical analysis was performed by $\mathrm{Peter} M$. Sweetnam at the MRC Epidemiology Unit, Cardiff. We are also to members of the Department of Medicine, University Hospital, Nottingham that assisted with the testing, and the Haematology department, University Hospital, Nottingham for the full blood counts performed.

\section{References}

Burgess-Wilson ME, Green S, Heptinstal! S and Mitchell JRA. Spontaneous Platelet Aggregation in Whole Blood: Dependence on age and haematocrit. Lancet, Nov 24 1984; ii: pp 1213.

Burgess-Wilson ME, Morrison R and Heptinstall S. Spontaneous platelet aggregation in heparinised blood during pregnancy. Thromb Res 1986; 37: 385-393.

Clinical Haematology, Fourth edition. Eastham RD, ed. John Wright \& Sons Ltd, Bristol, 1974.

Butchers J, Humphrey PPA, Hyde JJ, Lumley P and Spurling NW. The evaluation of a new electronic counting technique for measurement of platelet aggregation in human whole blood in vitro. Br J Pharmacol 1980: 70: 160-161.

Dawber TR. Incidence of coronary heart disease, stroke, and peripheral arterial disease. In: The Framingham Study. Harvard University Press, Cambridge, Máss, 1980, pp 59-75.

Dormandy JA. Haemorheology and thrombosis. In : Bloom AL, Thomas DP, eds. Ilaemostasis and thrombosis. Churchill Livingstone, Edinburgh, 1981, pp 610-625.

Fox SC, Burgess-Wilson M, Heptinstall S and Mitchell JRA. Platelet aggregation in whole blood determined using the Ultra-Flo 100 platelet counter. Thromb Haemost 1982; 48: 327 329.

Fulton RM and Duckett K. Plasma-fibrinogen and thromboemboli after myocardial infarction. Lancet 1976; ii: 1161-1164.

Gaarder A, Jonsen J, Laland S, Hellem A and Owren PA. Adenosine diphosphate in red cells as a factor in the adhesiveness of human blood platelets. Nalure 1961;192:531-532. 
Haines AP, Howarth D, North WRS, Goldenberg E, Stiring $\mathrm{Y}$, Meade TW, Raftery EB and Millar Craig MW. Haemostatic variables and the ontcome of myocardial infarction. Thromb Haemost 1983;50:800-803.

Harrison MJG and Mitchell JRA. The influence of red blood-cells on platelet adhesiveniess. Lancet $1966 ;$ ii: $163-64$.

Harrison MJG, Pollock SS and Weisblatt E. Haematocrit and platelet aggregation. Lancet 1984; ii: pp $901-992$

Hellem AJ, The adhesiveness of human blood platelets in vitro. Scand J Clin Lab Invest 1960; 12 (suppl 51 ).

Lowe GDO, Drummond MM, Lorimer AR, Hutton I, Forbes CD, Prentice CRM and Barbenel JC. Relation between extent of coronary artery disease and blood wiscosity. BMJ $1980 ; 280$ : $673-674$

Lowe GDO and Forbes CD. Platelet aggregation, haematocrit, and fibrinogen. Lancet 1985 Fob. 16, pp 395-396.

Lumley P and Humphrey PPA. A method for quantitating platelet aggregation and analysing drug-receptor interactions on platelets in whole blood in vitro. J Pharmacol Methods 1981; 6: $153-\| 66$

Meade TW, North WRS, Chakrabanti R, Stirling $Y$, Haines AP and Thompson SG. Haemostatic function and cardiovascular death: early results from a prospective study. Lancet $1980 ; 1: 1050-1054$.

Numn $\mathrm{B}$ and White IPN. Inhibitors of platelet aggregation have different activity in blood than in platelet-rich plasma. Br J Pharmacol 1980; 70:163-164.

Preston FE, Martin JF, Stewart RM and Davies-Jones GAB. Thrombocytosis, circulating platelet aggregates, and neurological dysfunction. BMJ $1979 ; 2 ; 1561-1563$.

Saniabadi AR, Lowe GDO, Barbenel JC, Forbes CD. Haematocrit, bleeding time, and platelet aggregation. Lancet 1984a; i: pp $1409 * 10$,

Saniabadi $A R$, lowe GDO, Barbenel IC and Forbes CD. A comparison of spontaneous platelet aggregation in whole blood with platelet rich plasma: Additional evidence for the role of ADP. Thromb Haemosi 1984b; 51:115-118. 


\section{CHAPTER 6}

\section{Platelet Aggregation in Whole Blood From Patients With Glanzmann's Thrombasthenia}

Burgess-Wilson ME, Cockbill SR, Johnston GI and Heptinstall S. Blood 1987, $69: 38-42$.

\section{Abstract}

We examined platelet aggregation in platelet-rich plasma (PRP) and in whole blood from two patients with Glanzmann's thrombasthenia. In PRP, aggregation was measured by monitoring the changes in light absorbance that occurred in response to aggregation agents: to measure platelet aggregation in whole blood, we used a platelet counting technique. In PRP, the patients' platelets showed defective aggregation in response to ADP, adrenaline, arachidonic acid (AA), and collagen, but normal agglutination occurred in response to ristocetin. In whole blood, however, platelet aggregation in response to the aggregating agents appeared to be either very simillar to that which occurred in blood from normal subjects or only slightly reduced. There was a reduced response to all concentrations of ADP and to low concentrations of collagen but a normal response to all concentrations of adrenaline, $\mathrm{A} A$, and higher concentrations of collagen. Conversely, there seemed to be an increased agglutination response to ristocetin. The abnormality in our two patients with Glanzmann's thrombasthenia probably lies in the inability of their platelets to form large, macroscopic aggregates rather than in platelet aggregation per se. 


\section{Introduction}

Glanzmann's thrombasthenia is a rare bleeding disorder characterised by defective platelet aggregation but with a normal platelet count (1). Platelets from patients with this condition either do not aggregate or aggregate very poorly in response to agents such as ADP, adrenaline, arachidonic acid (AA), and collagen. Platelets from such patients agglutinate normally in response to ristocetin $(2,3)$, and this has been used to aid the diagnosis of the condition. Defective platelet aggregation is believed to result from a markedly reduced amount of a complex of glycoproteins (the glycoprotein Ilb/IIla complex) on the platelet surface (4). This glycoprotein complex is essential for normal platelet aggregation but plays no part in ristocetin-induced platelet agglutination.

Previous studies in which platelet aggregation in Glanzmann's thrombasthenia was compared with platelet aggregation in healthy controls involved the use of platelet-rich plasma (PRP), in which aggregation is measured by following the changes in light absorbance that occur. With this technique, macroscopic platelet aggregates must form before the light absorbance of the PRP falls. It is now possible to measure platelet aggregation in whole blood by counting the number of single platelets (i.e. unaggregated platelets) using a whole-blood platelet counter $(5,6)$. Because the technique relies on the determination of the number of single platelets that remain in the blood rather than the size of the platelet aggregates that form, it is an extremely sensitive means of detecting platelet aggregates composed of relatively few platelets. In the present investigation, we compared platelet aggregation in PRP (measured with light absorbance) and whole blood (measured with the platelet counting technique) from patients with Glanzmann's thrombasthenia. The results obtained using whole blood were compared with those obtained for healthy controls.

\section{Materials and methods}

Materials. Adenosine 5'-diphosphate (ADP, sodium salt) adrenaline, and arachidonic acid (AA, grade 1 from porcine liver) were obtained from Sigma Chemical (St Louis). ADP and adrenaline were dissolved in saline before use; AA was converted to the sodium salt before use by dissolving in $0.1 \mathrm{~mol} / 1$ of sodium carbonate. Collagen (suspended equine collagen fibrils) was obtained from Hormon-Chemie and used as directed. Ristocetin sulphate was obtained from H. Lundbeck and dissolved in saline. Saline was $150 \mathrm{mmol} / 1$ of sodium chloride from Travenol Laboratories. The solution of citrate that was used to 
anticoagulate blood was $3.8(\mathrm{w} / \mathrm{v})$ trisodium citrate dihydrate. M148 (a monoclonal antibody directed against the glycoprotein IIb/IIa complex) was a gift from Professor R.M. Hardisty, Institute of Child Health, London, and was used at a final dilution of 1 in 2000. A control murine antibody 791T/36 (human osteogenic sarcoma cell-line) was a gift from Dr M. Price, Cancer Research Laboratories, University of Nottingham, England.

Subjects. Blood samples were taken from a sister and brother, designated as patient 1 and patient 2 throughout, aged 17 and 19 years, respectively, who had a similar history of mild bruising and bleeding. The diagnosis of Glanzmann's thrombasthenia had been made by Dr F.E. Preston (Department of Haematology, Royal Hallamshire Hospital, Sheffield, England.) on the basis of clinical history and the pattern of platelet aggregation in PRP characteristic of the condition. When examined, the patients exhibited prolonged bleeding times (30 and 21 minutes, respectively), but clot retraction was normal as were the whole blood platelet counts.

Blood samples were also taken from six healthy controls (three men and three women with an age range of 18 to 42 years) to obtain a set of normal values for platelet aggregation in whole blood. The tests on these samples were carried out using the same protocol as that used for the two patients.

Blood collection. Blood was collected with minimum venous stasis from the antecubital vein into polypropylene syringes using a 19 gauge, 2 -inch needle, and aliquots $(9 \mathrm{ml})$ were dispensed into polystyrene tubes that contained $1 \mathrm{ml}$ of citrate. Some of the citrated blood was centrifuged at $180 \mathrm{~g}$ for 10 minutes to prepare PRP, and the blood from which the PRP had been removed was then centrifuged at $1200 \mathrm{~g}$ for 20 minutes to obiain platelet-poor plasma. The latter was used to adjust the platelet count in the PRP to $300 \times 10^{9}$ platelets $/ 1$.

Measurements of platelet aggregation. Platelet aggregation in PRP was studied using the aggregometer and technique described by Adams and colleagues (7). Changes in light absorbance were recorded using a pen recorder.

Platelet aggregation in whole blood was assessed by determining the number of single platelets that remained in the blood after adding an aggregating agent to a sample. The number of single platelets present was counted using an Ultra-Flo 100 Whole Blood Platelet Counter and the technique described by Fox and coworkers (6). In brief, samples (500 $\mathrm{\mu l}$ ) of blood were placed in small polystyrene tubes and the tubes were placed in a water bath at $37^{\circ} \mathrm{C}$. After 2 minutes, a small aliquot of the blood was removed for platelet counting, a metal stirrer bar was placed in each tube, and the blood was stirred at $1000 \mathrm{rpm}$. After a further 2 minutes, another small aliquot was removed for platelet counting, and a solution 
of the aggregating agent under investigation was then added. Stirring was then continued, and further small aliquots were removed for platelet counting at 2 minute intervals up to 6 minutes after addition of the aggregating agent. The results obtained were expressed as the percentage of single platelets lost from the blood relative to the number of platelets present immediately prior to the addition of aggregating agent.

Inhibition of platelet aggregation by MI48. The monoclonal antibody, M148, binds to the glycoprotein IIb/IIIa complex on the surface of normal platelets and inhibits aggregation induced by ADP, adrenaline, AA, and collagen in both PRP and in whole blood (8). In some experiments, M148 (at a final dilution of 1 in 2000 ) was added to blood from the two patients with Glanzmann's thrombasthenia 2 minutes before the aggregating agent; aliquots were then removed for platelet counting at 20 second intervals up to 2 minutes after addition of the aggregating agent. To facilitate this quicker sub sampling technique, the aliquots $(15 \mu l)$ were taken into fixative as described by Bevan and colleagues (9) to be counted later on the Ultra-Flo 100. The results were compared with those obtained using a control murine antibody $(791 \mathrm{~T} / 36)$.

Mensurement of glycoprotein expression. Expression of the glycoprotein I $\mathrm{b} / \mathrm{Llla}$ complex on the surface of platelets from the patients was measured using the monoclonal antibody, M148 (10), combined with the technique of flow cytofluorimetry. After $240 \mu l$ samples of PRP were incubated with M148, aliquots containing $2 \times 10^{6}$ platelets were removed to tubes containing a fluorescent-labelled rabbit anti-mouse immunoglobulin as described previously (11). After a further incubation, immunofluorescence analysis was carried out using the FACSIV Flow Cytofluorimeter (12).

Microscopic examination of platelet aggregates. After samples of blood from both patients with Glanzmann's thrombasthenia and samples of blood from

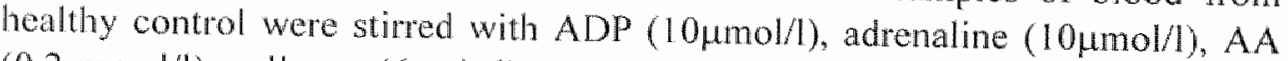
$(0.2 \mathrm{mmol} / \mathrm{l})$, collagen $(6 \mu \mathrm{g} / \mathrm{ml})$, and ristocetin $(2 \mathrm{mg} / \mathrm{ml})$, each for 6 minutes, the blood was smeared on a glass slide and stained with May-Grunwald-Giemsa stain. The tails of the blood smears were then examined for platelet / platelet aggregates under a light microscope. The effect of preincubating blood from the patients and controls with MI48 or control antibody prior to adding $5 \mu \mathrm{mol} / 1$ of ADP was also determined. 


\section{Results}

Platelet aggregation in PRP. The light absorbance of samples of PRP from the patients with Glanzmann's thrombasthenia either did not fall or fell only slightly when adrenaline, ADP, AA, or collagen was added to the PRP. In contrast, adding ristocetin to PRP from the patients induced a large fall in light absorbance; this was accompanied by the appearance of macroscopic platelet aggregates in the sample. Examples of the results obtained for the two patients are given in Fig 1, these results having been seen on more than one occasion.

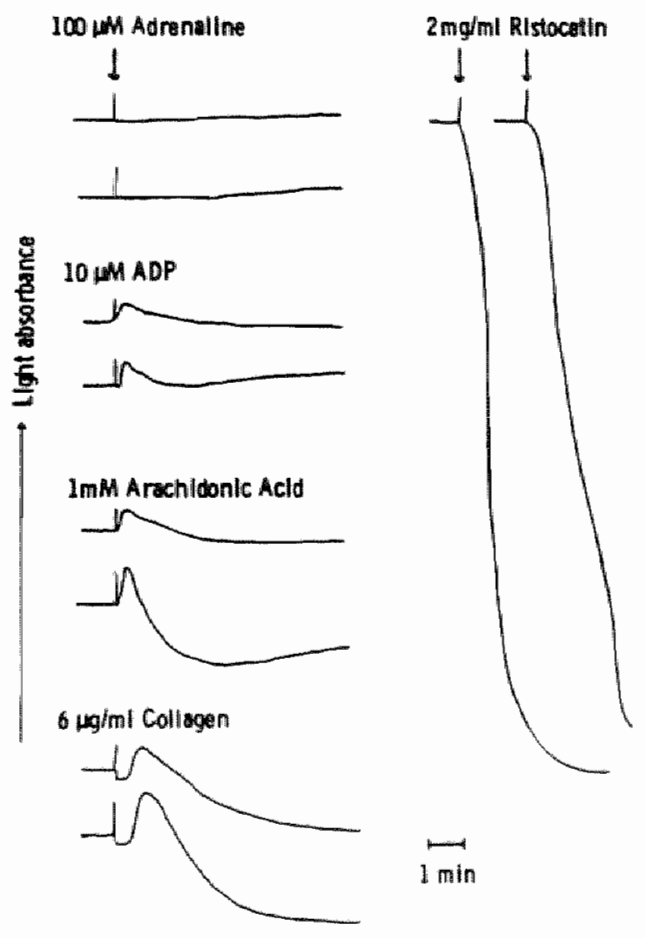

\section{Figure 1}

Fig. 1 The changes in light absorbance that occurred in platelet-rich plasma (PRP) $(480 \mu \mathrm{l})$ that had been stirred at $37^{\circ} \mathrm{C}$ for 2 minutes followed by the addition of various aggregating agents $(20 \mu 1)$. Typical traces obtained from both of the patients on one occasion are shown. For each aggregating agent the first of the pair of traces is that obtained from patient 2 and the second is that for patient $\mathbb{1}$. 
Platelet aggregation in whole blood. In contrast to the results obtained in PRP, platelet aggregation did occur when ADP, adrenaline, AA, or collagen was added to whole blood from the patients with Glanzmann's thrombasthenia. Table 1 shows the percentage loss of single platelets 6 minutes after the addition of aggregating agents to samples of whole blood obtained from the two patients and the controls. There was a reduced response in both patients, as compared with the mean control value, to all concentrations of ADP (Fig. 2) and to the lower concentrations of collagen $(0.3$ and $1.0 \mu \mathrm{g} / \mathrm{m} 1)$ and $\mathrm{AA}(0.1$ $\mathrm{mmol} / \mathrm{l}$ ) but a nomal response to all concentrations of adrenaline and higher concentrations of collagen ( 3 and $6 \mu \mathrm{g} / \mathrm{ml})$ and AA $(0.2 \mathrm{mmol} / \mathrm{l})$. There was an increased agglutination response to the lower concentration of ristocetin used.
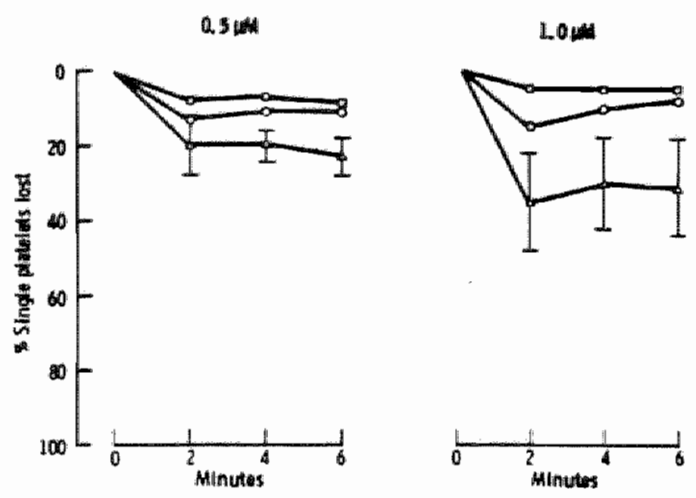

2. 9.4
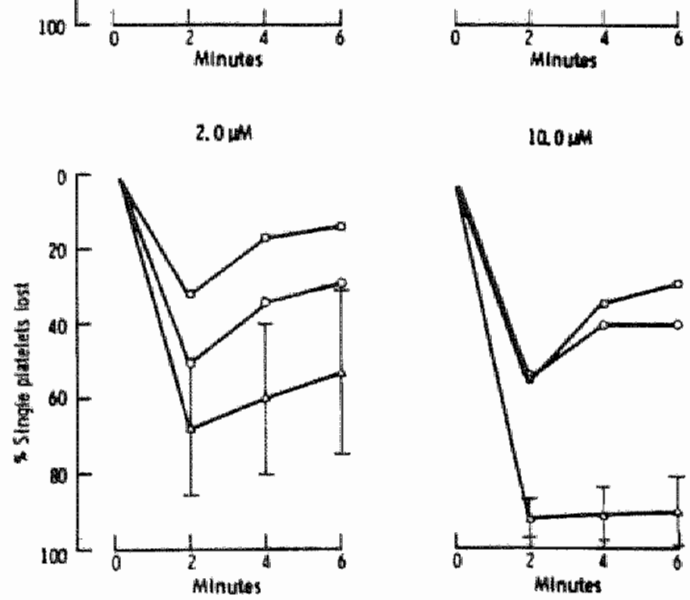

10.014

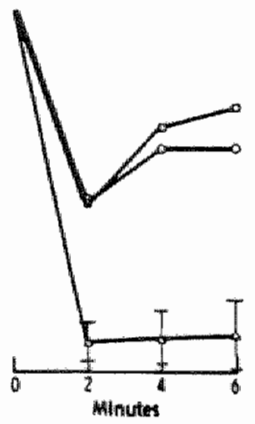

Figure 2

Fig. 2 The percentage of single platelets lost from whole blood stirred at $37^{\circ} \mathrm{C}$ following the addition of different concentrations of $\mathrm{ADP}$ at time 0 . The results shown are for patient $1(O)$ and patient $2(\square)$. The control values $(\Delta)$ are the mean $\pm S D(n=6)$. 
When samples of whole blood from the patients with Glanzmann's thrombasthenia were pre incubated with M148, the platelet aggregation induced by ADP, adrenaline, and collagen, was virtually abolished (Fig 3 ).
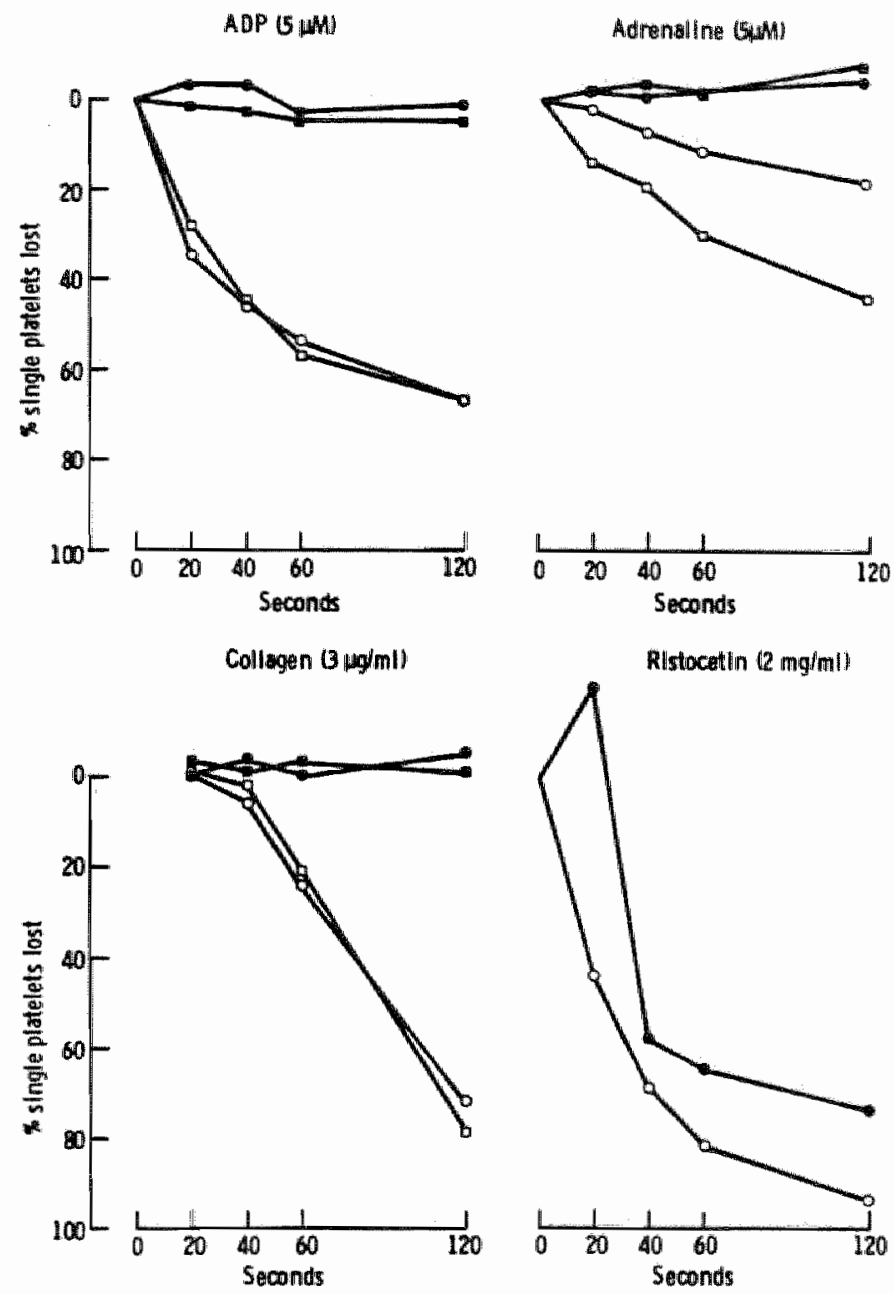

Figure 3

Fig. 3

The percentage of single platelets lost from whole blood stirred at $37^{\circ} \mathrm{C}$ following the addition of various aggregating agents at time 0 . The blood had been pre incubated for 2 minutes with either M148 or a control antibody. The results are for patient 1 in the presence of either $\mathrm{M} 148($ ) or control antibody $(\mathrm{O})$ and for patient 2 in the presence of either M148 ( ) or control antibody ( $\square$ ). Unfortunately, only data for patient 1 were obtained for ristocetin-induced agglutination. 
Glycoprotein expression. After immunofluorescence analysis of platelets incubated with M148 followed by a fluorescent-labelled immunoglobulin, the number of glycoprotein IIb/llla complexes on the surface of platelets from patients 1 and 2 were calculated to be 450 and 1600, respectively. This compares with a mean value of 17,300 for platelets from normal subjects (8). We have already shown that these patients have platelets that are deficient in glycoprotein $11 \mathrm{~b} / \mathrm{LII}$ a and that the deficiency does not reside in a sub population of cells (11).

Microscopy. Samples of blood to which aggregating agents had been added were examined for the presence of platelet aggregates by light microscopy. Platelet aggregates were present in whole blood the patients with Glanzmann's thrombasthenia as well as in blood from the normal controls. When blood from the patients or controls was incubated with M148 before adding ADP, only single platelets were seen.

\section{Discussion}

The results of our studies on platelet aggregation in PRP in which we used light absorption as a means of measuring the platelet aggregation that occurred are entirely consistent with those obtained by other workers who have investigated patients with Glanzmann's thrombasthenia (1-3). Aggregation was clearly defective in PRP from the two patients that we investigated. We were therefore surprised to find that the loss of single platelets induced by the addition of aggregating agents to whole blood from the patients appeared to be very similar to the loss obtained from healthy controls or was only slightly reduced. The reduced response to ADP was greater than ISD at lower concentrations and greater than $2 \mathrm{SD}$ 's at the highest concentration $(10 \mu \mathrm{mol} / 1)$, whereas the reduced responses to collagen and $\mathrm{AA}$ were only seen at the lower concentrations tested.

By preincubating whole blood from the patients with M148, we were able to confirm that the single platelets that were lost when an aggregating agent was added to whole blood were indeed taking part in aggregate formation. The addition of this monocional antibody virtually abolished the platelet loss that occurred following addition of ADP, adrenaline, or collagen to blood from the patients with Glanzmann's thrombasthenia. As further confirmation that platelet aggregation was occurring in whole blood, we looked directly for platelet aggregates in blood from the patients using light microscopy. Platelet aggregates were observed in all the samples to which an aggregating agent had been added. In addition, M148 prevented aggregate formation. 


\section{Table 1}

Percentage of single platelets lost six minutes after addition of aggregating agents to samples of whole blood stirred at $37^{\circ} \mathrm{C}$.

\begin{tabular}{|l|c|c|l|}
\hline Aggregating agent & Patient 1 & Patient 2 & Controls \\
\hline Saline & 2 & 4 & $10 \pm 6$ \\
\hline ADP $(0.5 \mu \mathrm{mol} / \mathrm{l})$ & 9 & 8 & $22 \pm 5$ \\
\hline ADP $(1.0 \mu \mathrm{mol} / \mathrm{l})$ & 4 & 6 & $30 \pm 14$ \\
\hline ADP $(2.0 \mu \mathrm{mol} / \mathrm{l})$ & 26 & 15 & $52 \pm 22$ \\
\hline ADP $(10.0 \mu \mathrm{mol} / \mathrm{l})$ & 40 & 28 & $90 \pm 9$ \\
\hline Adrenaline $(0.2 \mu \mathrm{mol} / \mathrm{l})$ & 16 & 10 & $10 \pm 7$ \\
\hline Adrenaline $(0.5 \mu \mathrm{mol} / \mathrm{l})$ & 18 & 19 & $16 \pm 9$ \\
\hline Adrenaline $(1.0 \mu \mathrm{mol} / \mathrm{l})$ & 21 & 25 & $20 \pm 9$ \\
\hline Adrenaline $(2.0 \mu \mathrm{mol} / 1)$ & 24 & 38 & $26 \pm 10$ \\
\hline Adrenaline $(5.0 \mu \mathrm{mol} / 1)$ & 30 & 39 & $33 \pm 17$ \\
\hline AA $(0.1 \mathrm{mmol} / 1)$ & 4 & 2 & $43 \pm 33$ \\
\hline AA $(0.2 \mathrm{mmol} / 1)$ & 92 & 93 & $95 \pm 3$ \\
\hline Collagen $(0.3 \mu \mathrm{g} / \mathrm{ml})$ & 11 & 7 & $20 \pm 14$ \\
\hline Collagen $(1.0 \mu \mathrm{g} / \mathrm{ml})$ & 31 & 18 & $80 \pm 29$ \\
\hline Collagen $(3.0 \mu \mathrm{g} / \mathrm{ml})$ & 89 & 90 & $96 \pm 1$ \\
\hline Collagen $(6.0 \mu \mathrm{g} / \mathrm{ml})$ & 92 & 94 & $97 \pm 1$ \\
\hline Ristocetin $(1.0 \mathrm{mg} / \mathrm{ml})$ & 96 & 99 & $20 \pm 13$ \\
\hline Ristocetin $(2.0 \mathrm{mg} / \mathrm{ml})$ & 97 & 99 & $95 \pm 2$ \\
\hline
\end{tabular}

We consider that the apparent discrepancy between results obtained in whole blood and PRP in this investigation relates to the differences in the two techniques that were used to measure the aggregation that occurred. The light absorbance of PRP falls only when very large platelet aggregates (that are visible to the naked eye and must contain many thousands of platelets) form. Conversely, the number of single platelets in whole blood falls as soon as the single platelets take part in aggregate formation whatever the size of the aggregates that develop. The defective platelet aggregation in PRP from patients with Glanzmann's thrombasthenia may occur because the aggregates that form are relatively small. We believe that it is unlikely that the different 
results in PRP and whole blood relate to the presence of $\mathrm{RBCs}$ in the latter, but this possibility has not been excluded.

Although the patients we investigated had platelets that contained markedly reduced amounts of surface-expressed glycoprotein $\mathrm{Ilb} / \mathrm{III}$ a complex ( $3 \%$ and $9 \%$ of normal for patients 1 and 2 , respectively), the platelets were not completely devoid of the glycoprotein complex. In addition, clot retraction occurred in coagulated whole blood from these patients. Thus, according to the definition of Caen (13), our patients probably have type II Glanzmann's thrombasthenia. Platelets from patients with type I Glanzmann's thrombasthenia have undetectable amounts of the glycoprotein complex and elot retraction is absent in coagulated blood.

Although Nurden and colleagues (14) recently reported the presence of measurable amounts of glycoprotein IIb and/or glycoprotein IIIa on the platelets of patients with type \Glanzmann's thrombasthenia, they proposed that it was the number of functional glycoprotein IIb/IIIa complexes expressed on the surface of the platelet that was important in discriminating between the two subgroups. It is probable that the small amounts of complex that are present on our patients" platelets allow partial platelet aggregation that is detectable in our whole blood system but not in PRP. In view of the reduced platelet aggregation that occurs in response to ADP and low concentrations of collagen in whole blood from these patients, it will be interesting to discover if platelet aggregation is further reduced in whole blood from patients with a more severe glycoprotein Ilb/IIIa complex deficiency. It must be noted that in our investigation platelets from patient 1 aggregated slightly more extensively in both PRP and whole blood than did platelets from patient 2 , despite having a lesser amount of the glycoprotein IIb/IIla complex available and a longer bleeding time. This lack of correlation between glycoprotein expression, platelet aggregation, and bleeding time in these two patients may be caused by the close similarity of the patients with regard to both clinical and laboratory parameters, however.

In the cases of Glanzmann's thrombasthenia we have studied, only relatively small amounts of glycoprotein IIb/IIIa were expressed, and the deficiency resides in all the platelets rather than in a sub population (11). We have suggested that it is the amount of glycoprotein IIb/IIIa on the surface of platelets that determines the size of the aggregates that are produced in response to an aggregating agent and that the true deficiency in Glanzmann's thrombasthenia relates to the size of the aggregates produced rather than to aggregation per se. The precise relationship between aggregate size and 
glycoprotein IIb/IIIa expression needs to be explored by further experimentation.

\section{Acknowledgements}

We are grateful to Dr. F.E.Preston for kindly allowing us to study his patients, and to Professor R.M. Hardisty and Dr M. Price for gifts of antibodies.

\section{References}

1. Hardisty RM. Disorders of platelet function. In: Human Blood Coagulation, Haemostasis and Thrombosis. Biggs R and Rizza CR, eds. Blackwell Scientific Publications, Oxford, 1984; pp 349.

2. Hardisty RM, Dormandy KM and Hutton RA. Thrombasthenia: Studies on three cases. Br J Haem 1964; 10: 371.

3. Howard MA and Firkin BG: Ristocetin: $A$ new tool in the investigation of platelet aggregation. Thromb Haemostas 1971; 26: 362.

4. George JN, Nurden AT and Phillips DR. Molecular defects in interactions of platelets with the vessel wall. N Eng J Med 1984; $3 \| 1$ : 1084.

5. Lumley P and Humphrey PPA. A method for quantitating platelet aggregation and analysing drug-receptor interactions on platelets in whole blood in vitro. I Pharmacol Methods 1981; 6: 153-166.

6. Fox SC, Burgess-Wilson $M$, Heptinstall $S$ and Mitchell JRA. Platelet aggregation in whole blood determined using the Ultra-Flo 100 platelet counter. Thromb Haemostas $1982 ; 48: 327-329$.

7. Adams J, Heptinstall S and Mitchell JRA. A six-channel automated platelet aggregometer. Thromb Haemostas 1975; 34: 821 .

8. Johnston GI. An examination of the role of calcium ions and membrane glycoproteins in blood platelet behaviour. PhD Dissertation 1985, University of Nottingham, England.

9. Bevan J and Heptinstall S. Serotonin-induced platelet aggregation in whole blood and the effects of ketanserin and mepyramine. Thromb Res 1985; 38: 189.

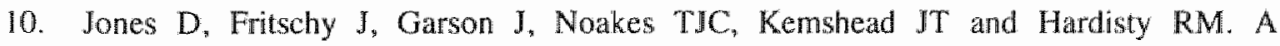
monoclonal antibody binding to human medulloblastoma cells and the platelet glycoprotein IIb-IIIIa complex. Br J Haem 1984; $57: 621$. 
11. Johnston GI, Heptinstall S, Robins RA and Price MR. The expression of glycoproteins on single blood platelets from healthy individuals and from patients with congental bleeding disorders. Biochem Biophys Res Commun 1984; 123: 1091.

12. Johnston $\mathrm{Gl}$ and Heptinstall $\mathrm{S}$. Examination of glycoproteins on intact blood platelets by using monoclonal antibodies and the fluorescence activated cell sorter. Biochem Soc Trans 1985; 13:109.

13. Caen J. Glanzmann's thrombasthenia. In: Clinics in Haematoloy, Vol 1. O'Brien JR, ed. Saunders, London, 1972 , pp 383.

14. Nurden AT, Didry D, Kieffer N and McEver RP. Residual amounts of glycoproteins IIb and IIra may be present in the platelets of most patients with Glanzmann's thrombasthenia. Blood 1985; 65: 1021. 


\title{
CHAPTER 7
}

\section{Diamide induces reversible aggregation of human blood platelets}

Lösche W, Burgess-Wilson M, Michel E, Heptinstall H and Till U. Thromb Res $1985,40: 869-874$.

\begin{abstract}
The results of this investigation emphasise the sensitivity of platelet counting techniques for measuring platelet aggregation compared with light transmission. They show that the GSH-depleting agent diamide can induce platelet aggregation in whole blood and PRP without simultaneous addition of another aggregating agent, and indicate that endogenous ADP contributes to the aggregation that is obtained.
\end{abstract}




\section{Introduction}

When compounds that oxidise reduced glutathione (GSH) to GSSG such as diamide (azodicarboxylic acid-bis-dimethylamide), cumene hydroperoxide or tertiary butyl hydroperoxide, are added to platelet-rich plasma together with an aggregating agent, e.g. adenosine diphosphate (ADP), collagen, thrombin or arachidonic acid, they modify the aggregation response. They accelerate the rate at which the platelets aggregate (as judged by the rate at which light transmission through the sample increases), and convert aggregation that would have been irreversible to reversible aggregation $(1,2)$. The altered aggregation is believed to result from the altered cellular thiol-disulfide status $(3,4)$.

Platelet aggregation can be measured by counting the number of single platelets in PRP as the aggregation proceeds. This is a much more sensitive means of detecting platelet aggregation than the measurement of light transmission. In addition, platelet counting can be used to measure platelet aggregation in whole blood. In this investigation we have used platelet counting techniques to show that diamide induces platelet aggregation directly in PRP and in whole blood without the addition of another aggregating agent. We also show that ADP from the platelets and/or red cells contributes to the aggregation that occurs.

\section{Material and methods}

Blood collection. Blood was collected from the anticubital vein of healthy volunteers into $1 / 10$ volume of $3.8 \%(w / v)$ trisodium citrate dihydrate. PRP was prepared by centrifugation of the citrated blood (CB) at $200 \mathrm{~g}$ for 15 minutes at room temperature and stored in a capped plastic tube in order to prevent escape of carbon dioxide and a subsequent rise in $\mathrm{pH}$.

Platetet aggregation. Platelet aggregation in $\mathrm{CB}$ was measured at $37^{\circ} \mathrm{C}$ in 0.5

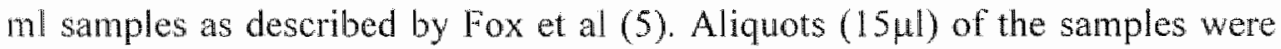
removed before and at different times after stirring the samples with cylindric $4 \times 1 \mathrm{~mm}$ stainless steel stirring bars at $1000 \mathrm{rpm}$. and mixed with $30 \mathrm{ul}$ formaldehyde/EDTA solution according to Wu and Hoak (6). After diluting 36 $\mu l$ of the fixed aliquots with $9.1 \mathrm{ml} 150 \mathrm{mM} \mathrm{NaCl}$ (saline) the number of single platelets was determined using the Ultra-Flo 100 Whole Blood Platelet Counter. Platelet aggregation in PRP was performed using the ELV1 840 aggregometer. In addition to the light transmission measurements, aliquots of the stirred PRP $\left(900 \mathrm{rpm}, 37^{\circ} \mathrm{C}\right)$ were removed, fixed with $1.2 \%(\mathrm{w} / \mathrm{v})$ glutaraldehyde and the 
number of single platelets was determined using a light microscope as described previously (7).

In some experiments $300 \mu \mathrm{M}$ creatine phosphate $(\mathrm{CP})$ and $11 \mathrm{u} / \mathrm{ml}$ creatine phosphokinase (CPK) were added to the samples of $C B$ and PRP two minutes before they were stirred. This is a substrate/enzyme system that destroys any ADP in the preparation.

\section{Results and discussion}

When either CB or PRP is stirred for up to 10 minutes there is a continuous fall in the number of single platelets that are present. Diamide $(1.0$ and $0.5 \mathrm{mM}$ respectively) increased the rate of this spontaneous platelet aggregation (Figure 1). The effect of the diamide was only transient and within 10 minutes the number of single platelets had returned to the value in the control tube.

Despite a steady fall in the number of single platelets in stirred PRP the amount of light transmitted through the sample did not change (Figure 2). Adding 0.1 $\mathrm{mM}$ diamide to stirred PRP produced a rapid fall (compared with the saline control) in the number of single platelets present, but again this was without any change in the amount of light transmitted through the sample. When $0.5 \mathrm{mM}$ diamide was added to PRP there was a very marked fall in the number of single platelets present and this was accompanied by a very slight increase in light transmission (Figure 2). The results of this experiment demonstrate the much greater sensitivity of counting techniques for the measurement of platelet aggregation compared with conventional aggregometry.

ADP from platelets and/or red cells was shown to contribute to the spontaneous aggregation in whole blood and PRP when the sample is stirred without any agent being added $(5,8)$. Experiments that were performed in the presence of $\mathrm{CP}$ and CPK demonstrated that ADP also contributes to the aggregation that is obtained when diamide is added to CB or PR.P. It can be seen (Figure 3 ) that the effect of the combination of $C P$ and $C P K$ was to reduce the extent of aggregation that occurred on stirring $\mathrm{CB}$ and PRP, and that it also reduced the extent of the response to diamide. 

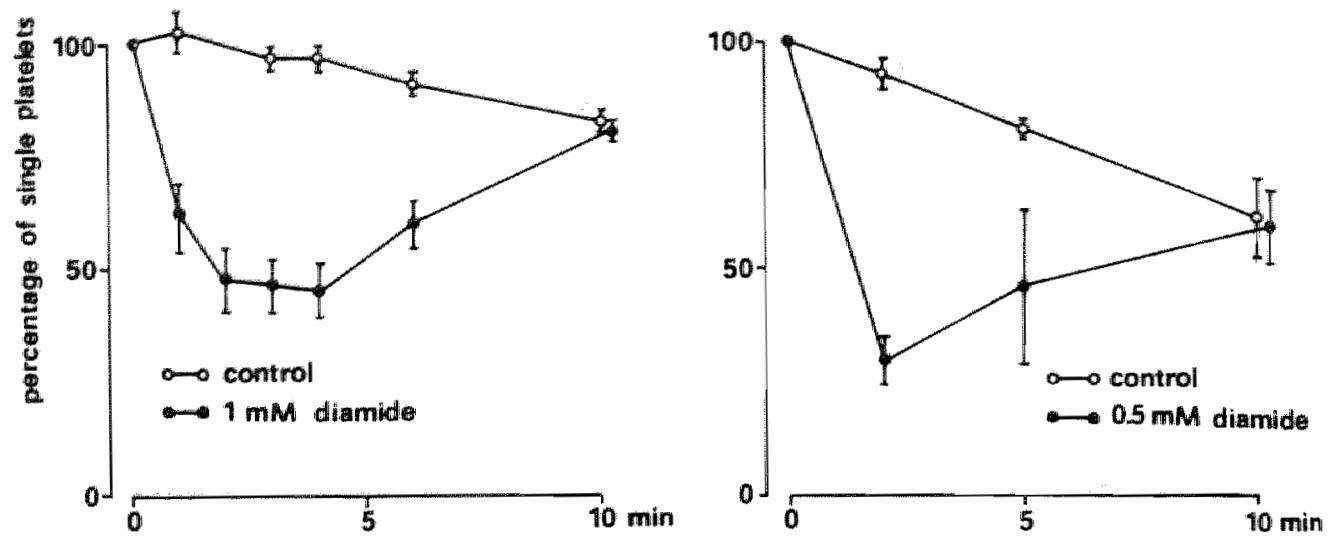

\section{Figure 1}

Fig. 1 The effect of diamide on the number of single platelets remaining in samples of $\mathrm{CB}$ (left) and PRP (right) when the samples were stirred at $37^{\circ} \mathrm{C}$. Diamide or saline (as control) was added immediately after the start of stirring. Values are mean 1 SEM. of results obtained for ten samples of CB and six samples of PRP from different individuals.

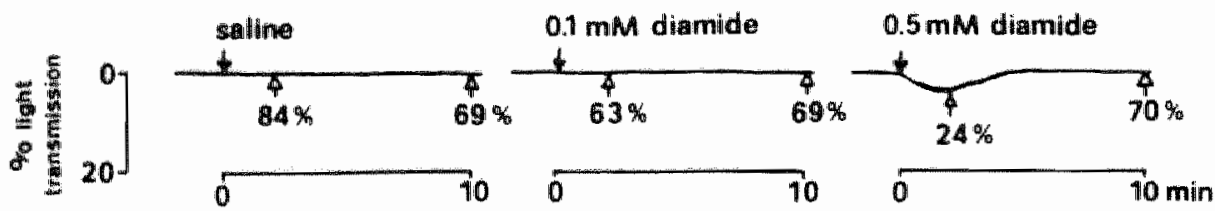

\section{Figure 2}

Fig. 2 Examples of the changes in light transmission that occurred when diamide for saline as control) was added to PRP. The numbers of single platelets present (percentage of the number before the diamide or saline was added) are also given. The results are representative of those obtained in three separate experiments 


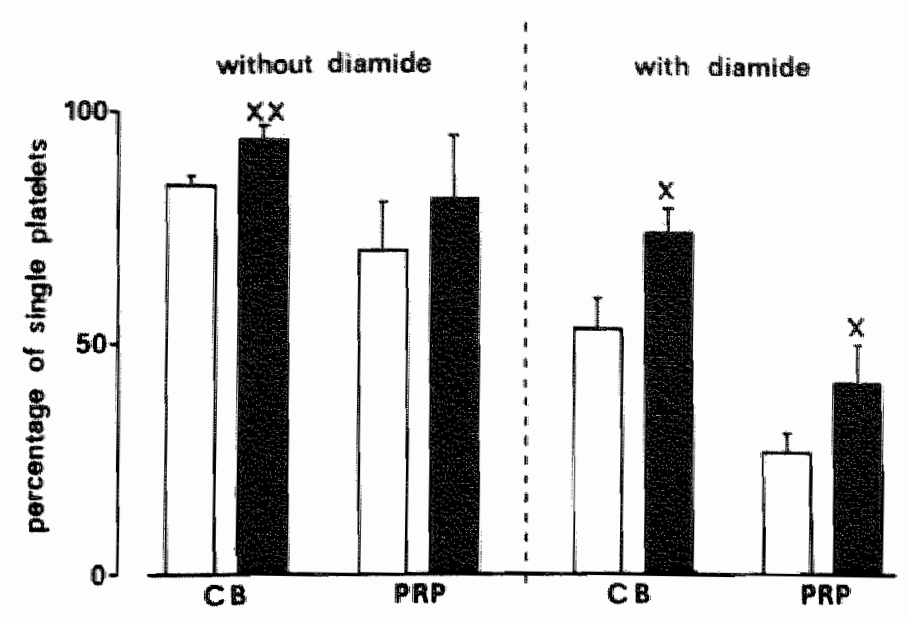

\section{Figure 3}

Fig. 3 The effects of CP/CPK on the number of single platelets in $C B$ and PRP that were stirred in the absence and presence of diamide. The concentration of diamide were 1.0 $\mathrm{mM}$ (CB) and $0.5 \mathrm{mM}$ (PRP). Platelet counts were performed after stirring the samples for 10 minutes (when diamide was absent), after four minutes (when diamide was added to CB) or after two minutes (when diamide was added to PRP). Values are mean \pm SEM. of results obtained for four samples of CB or PRP from different individuals, ( $\square$ ) without CP/CPK; (E) with $C P / C P K$. The significance of the effect of CP/CPK is indicated by: $x$ represents $p<0.05$; $\mathbf{x x}$ represents $p<0.001$ (Student's paired t-test).

However, it is not clear whether the diamide makes the platelets more sensitive to the ADP that is present or whether it encourages platelet aggregation by enhancing ADP liberation. Diamide is known to alter the properties of red cell membranes (9) and this could lead to increased liberation of ADP from these cells. Furthermore, diamide accelerates the platelet release reaction and thereby the rate at which ADP is liberated from the platelets (10). On the other hand, diamide increases the rate at which platelets aggregate in PRP when ADP and diamide are added simultaneously (2).

The results of this investigation emphasise the sensitivity of platelet counting techniques for measuring platelet aggregation compared with light transmission. They show that the GSH-depleting agent diamide can induce platelet aggregation in whole blood and PRP without simultaneous addition of 
another aggregating agent, and indicate that endogenous ADP contributes to the aggregation that is obtained.

\section{Acknowledgements}

This study was supported by the British Council and by the "Ministerium fur Hoch-und Fachshulwesen" and the "Hauptforschungsrichtung Hypertonie und ischämische Herzkrankheiten", GDR. The authors are also grateful to CORDA for a research grant and to $\mathrm{Mrs}$. $\mathrm{H}$. Schmied for typing the manuscript.

\section{References}

1. Patscheke $H$ and Womer P. Sequential effect of the thio-oxidizing agent, diamide, on human platelets. Thromb Res 1978; 12: 609-618.

2. Hofmatn J, Lösche W, Hofmann B, Arese P, Bosia A, Pescarmona GP and Till U. Effect of compounds causing reversible perturbation of cellular thiol-disulfide status on the aggregation of human blood platelets. Biomed Biochim Acta 1983; 42: 479-488.

3. Lösche W, Bosia A, Caruso B, Spangenberg P, Pescarmona GP, Galli G, Arese P, Paoletti $R$ and Till $U$. Dependence of arachidonic acid metabolism in human blood platelets on reduced coenzymes. Biomed Biochim Acta 1984; 43: S362-\$365.

4. Spangenberg P, Heller R, Bosia A, Arese P and Till U. Chemical modification of cytoskeletal proteins of human blood platelets by diamide. Thromb Res 1984;36: 609618.

5. Fox SC, Burgess-Wilson M, Heptinstall S and Mitchell JRA. Platelet aggregation in whole blood determined using the Ultra-Flo 100 Platelet Counter. Thromb Haemostas 1982; 48: 327-329.

6. Wu KK and Hoak IC. A new method for the quantitative detection of platelet aggregates in patients with arterial insufficiency. Lancet 1974;2:924-926.

7. Hofnann B, Danz R, Hofmann I, Pescarmona GP, Bosia A, Meyer M, Lösche W and Till U. Differences in morphology and protein pattern of human blood platelets during irreversible and diamide mediated reversible aggregation. Biomed Biochim Acta 1983; 42: $489-501$.

8. Samiabadi AR, Lowe GDO, Barbenel JC and Forbes CD. Acomparison of spontaneous platelet aggregation in whole blood with platelet rich plasma: additional evidence for the role of ADP. Thromb Haemostas 1984; 51: 115-118. 
9. Fischer TM, Haest CWM, Stohr M, Kamp D and Deuticke B. Selective alterations of erythrocyte deformability by SH-reagents: evidence for an involvement of spectrin in membrane shear elasticity. Biochim Biophys Acta 1978, 691:270-282.

10. Hofmann $J$, Losche $W$, Ostermann $G$ and Till $U$. Interdependence of GSH and formation of malondialdehyde in arachidonic acid induced platelet activation. In: Prostaglandins and Thromboxanes. W. Förster, ed. G. Fischer-Verl. Jena, 1981; pp. 197-198. 


\title{
CHAPTER 8
}

\section{The lack of effect of fish oil supplementation on whole blood platelet aggregation despite changes in platelet fatty acid composition.}

\begin{abstract}
Optical aggregation methods have been used in many studies to investigate the effect of fish oil ingestion on the function of platelets. The results however are equivocal, and it is possible that platelet-rich plasma is not a suitable medium for the detection of the changes in platelet behaviour associated with fish oil ingestion. This study investigated the changes in whole blood platelet aggregation when 5 normal healthy males were given fish oil over a 6 week period. As expected, the platelet eicosapentaenoic acid content increased with a concurrent reduction in the arachidonic acid levels. The changes in other fatty acids were comparatively small. Thromboxane $B_{2}$ production in clotted blood was reduced after ingestion which reflected these changes in fatty acid composition. There was, however, no significant change in the extent of whole blood platelet aggregation measured, or in any of the other tests of platelet activity used; platelet release reaction using platelet-rich plasma, platelet calcium and magnesium content and membrane glycoprotein (GPIb and GPIIb/IIIa) expression.
\end{abstract}

These results support the lack of effect seen in other studies using optical aggregation and impedance measurements in whole blood, and suggest that the beneficial effects on the incidence of heart disease, due to fish oil ingestion may not be caused by changes in platelet behaviour. It is possible, however, that the platelet tests used in this study were also unsuitable for the detection of any changes in platelet behaviour due to fish oil ingestion. 


\section{Introduction}

There is evidence that in populations where the diet is based on fish, there is a lower incidence of ischaemic heart disease (Dyerberg and Bang 1979, Kronman and Green 1980, Hirai et al 1980). Fish oils contain greater amounts of polyunsaturated fatty acids, especially eicosapentaenoic acid (EPA) than dairy products. Dyerberg and Bang (1979) have shown that fish-eating populations, such as Greenland Eskimos, have a different platelet fatty acid composition; having more EPA and less arachidonic acid (AA). AA is the precursor of thromboxane $A_{2}$, a potent platelet aggregating agent, whereas EPA, is metabolised to thromboxane $A_{3}$, which does not cause platelets to aggregate (Needleman et al 1979, Hamberg 1980). This difference in fatty acid composition may have the effect of reducing platelet activity in wivo, thus reducing the risk of thrombosis.

It is possible to supplement the diet with fish oil and increase the ratio of EPA to $\mathrm{A} A$ in the tissues and many studies have been performed using optical aggregation methods to investigate the effect of fish oil ingestion on the function of platelets. These studies have provided some evidence to suggest that in platelets the changes in fatty acid composition result in a change in platelet behaviour. But the evidence is conflicting and inconclusive (Hornstra 1989). It is possible that platelet-rich plasma (PRP) is not a suitable medium for the detection of the changes in platelet behaviour associated with fish oil ingestion. The preparation of PRP with the removal of red blood cells and white blood cells may influence the platelet activity measured. Conversely, whole blood platelet aggregation (WBA) may reveal aspects of platelet behaviour that are undetectable using PRP.

In this study the fish oill preparation MaxEPA was used and platelet behaviour was investigated before, and after 6 weeks ingestion, in a group of normal individuals.

\section{Materials}

MaxEPA was obtained from Seven Seas Health Care Ltd. Fatty acids and their methyl esters, adenosine 5'-disphosphate (ADP), adrenaline, and serotonin were from the Sigma Chemical Co. Ltd. Collagen was obtained from Hormon Chemie and ristocetin from $\mathrm{H}$. Lundbeck and Co. Prostacyclin was a gift from Dr. John Pike of Upjohn. Arachidonic acid was dissolved in $0.1 \mathrm{M}$ sodium carbonate before used. All solvents were of HPLC grade. 


\section{Methods}

Subjects tested. Five male volunteers took part in the study (age range 33-37 years). All were healthy and denied taking any medication in the two weeks before, and during the study. For each volunteer two blood samples were taken before fish oil ingestion (MaxEPA, 20ml daily for 6 weeks) and two further samples were taken at the end of the 6 week period.

Blood Collection. Blood was collected by clean venepuncture into at polypropylene syringe using a 19 gauge 2 " needle. Immediately after collection aliquots were transferred to polystyrene tubes (capacity $10 \mathrm{ml}$ ) that contained either $3.8 \%(\mathrm{w} / \mathrm{v})$ trisodium citrate dihydrate $(\mathrm{ml})$ or 100 IU heparin $(20 \mu \mathrm{L}$ of the stock solution in $180 \mu \mathrm{l}$ of $0.9 \% \mathrm{NaCl}$ ). Aliquots were also transferred to a tube that contained $6.4 \mathrm{mg}$ potassium EDTA $(4 \mathrm{ml}$ blood), a tube containing $0.4 \mathrm{ml}$ of $100 \mathrm{mM}$ EDTA disodium salt $(9.6 \mathrm{ml}$ blood), and an empty glass tube $(10 \mathrm{ml}$ blood $)$ in which the blood clotted.

Platelet aggregation in whole blood. Platelet aggregation in whole blood was measured using the method previously described by Bevan and Heptinstall (1985), using the fixing technique. The platelet count of the citrated or heparinised blood was measured, then the blood stirred for 2 minutes and the platelet count measured. If appropriate the aggregating agent was then added, and the platelet count was measured after another 2, 4 and 6 minutes stirring. Aggregation was measured in response to stirred alone (called spontaneous platelet aggregation, SPA) using both citrated and heparinised whole blood samples. Platelet aggregation in response to the platelet agonists ADP $(0.5 \mu \mathrm{M})$ and collagen $(1 \mu \mathrm{g} / \mathrm{ml}$ to $3 \mu \mathrm{g} / \mathrm{ml})$ was measured using only citrated blood.

Platelet release reaction in platelet-rich plasma. The extent of the release reaction that occurred in response to various aggregating agents was determined using citrated PRP in which the platelets had been labelled with ${ }^{14} \mathrm{C}$-serotonin as previously described (Jones et al 1983). The aggregating agents used were arachidonic acid $(0.1 \mathrm{mM}$ to $1 \mathrm{mM})$, ADP $(10 \mu \mathrm{M})$, adrenaline $(0.1 \mu \mathrm{M}$ to $100 \mu$ M), collagen $(0.3 \mu \mathrm{g} / \mathrm{ml}$ to $6 \mu \mathrm{g} / \mathrm{ml})$ and ristocetin (1 and $2 \mathrm{mg} / \mathrm{ml}$ ). The sensitivity of the platelets to the inhibitory effects of dazoxiben, a thromboxane synthetase inhibitor (Jones et al 1983), and to prostacyclin (Andrews et al 1985) were also determined.

Assay of platelet calcium and magnesium. The intra-platelet calcium and magnesium levels were measured in platelets as previously described (Heptinstall 1976). 
Platelet fatty acid analysis. The fatty acid composition of the platelet was measured as described by Cockbill et al (1985) using the methods of Bligh (1959) and Glass (1971).

Thromboxane $B_{2}$ measurements. The amount of thromboxane $\mathrm{B}_{2}\left(\mathrm{TxB}_{2}\right)$ produced in clotted whole blood was measured as described previously (Carter and Hanley 1985).

Measurement of glycoprotein expression. The amounts of glycoprotein Ib and the glycoprotein complex IIb/IIIa expressed on the surface of individual platelets was measured as previously described by Johnston and Heptinstall (Johnston and Heptinstall 1985).

Haematological parameters. A full blood count was made using the $4 \mathrm{ml}$ EDTA sample. Testing was performed in the Haematology Department, University Hospital Nottingham using the Coulter S-IV.

\section{Results}

The ingestion of fish oil changed the fatty acid composition of the platelets (Table 1). The platelet EPA content increased and the AA content fell.

Table 1 The percentage distribution (by weight) of platelet fatty acids before and after 6 weeks ingestion of fish oil (EPA)

\begin{tabular}{|c|c|c|c|}
\hline Fatty Acid & $\begin{array}{c}\text { Before } \\
\text { Mean (SEM) }\end{array}$ & $\begin{array}{c}\text { After 6 weeks } \\
\text { Mean (SEM) }\end{array}$ & p \\
\hline $16: 0$ & $16.5(0.8)$ & $15.4(0.5)$ & - \\
\hline $18: 0$ & $12.7(0.6)$ & $12.1(0.8)$ & - \\
\hline $18: 1$ & $16.5(0.5)$ & $15.8(0.5)$ & - \\
\hline $18: 2$ & $13.6(1.9)$ & $12.5(1.2)$ & - \\
\hline $20: 0$ & $1.0(0.1)$ & $0.9(0.1)$ & - \\
\hline $20: 4$ & $23.9(1.8)$ & $18.5(2.0)$ & 0.01 \\
\hline $20: 5$ & $1.8(0.6)$ & $9.4(1.2)$ & 0.002 \\
\hline Unknown & $13.8(1.3)$ & $14.5(1.8)$ & - \\
\hline
\end{tabular}




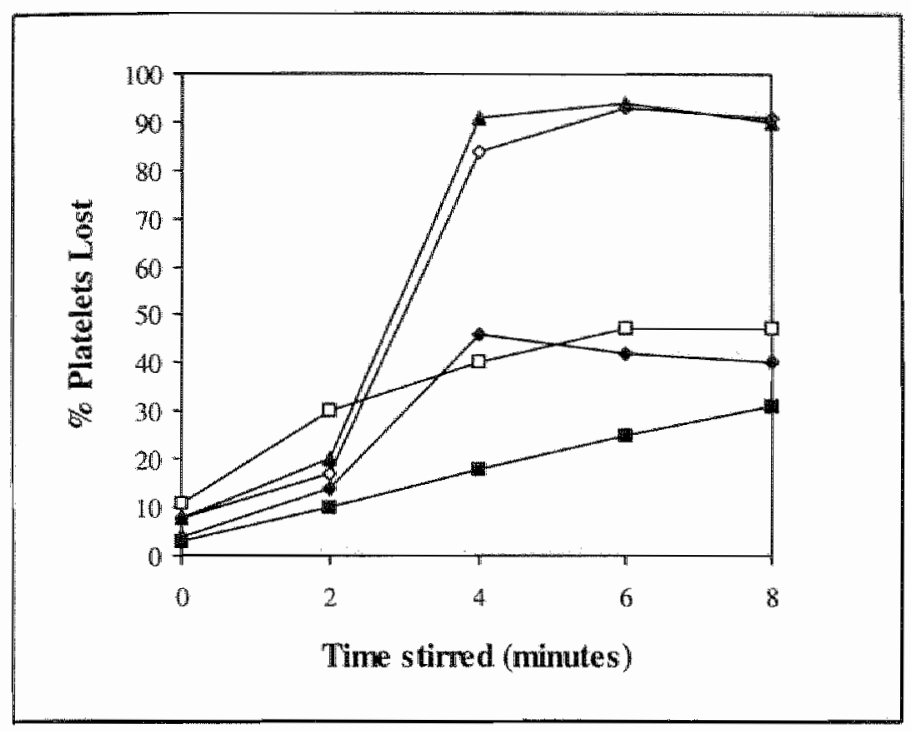

Figure 1

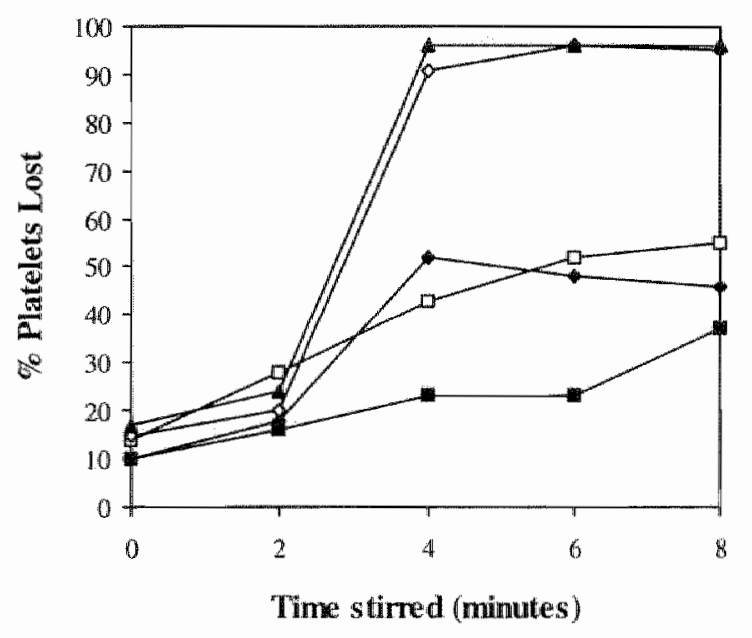

\section{Figure 2}

Figs. 1 and 2 The mean number of single platelets $\operatorname{lost}(n=5)$, before (Fig. 1) and after (Fig. 2) 6 weeks of fish oil ingestion, either as SPA in citrated (w) or heparinised blood ( $\square$ ), or in response to an aggregating agent, $0.5 \mu \mathrm{M} \mathrm{ADP}(\diamond), 1 \mathrm{mg} / \mathrm{l}$ Collagen ( 4 ) or $3 \mathrm{mg} / \mathrm{l}$ Collagen ), added after 2 minutes stirring. 
There was a significant increase $(p=0.01)$ in the platelet content of $20.5 \mathrm{w} 3$ following fish oil administration and a significant reduction $(\mathrm{p}=0.002)$ in $20: 4 w 6$. The ratio of $20: 5 / 20: 4$ changed significantly ( $p=0.01$ ) from 0.08 (SEM $\pm 0.02)$ to $0.56(\mathrm{SEM} \pm 0.12)$.

This alteration in the fatty acid profile was reflected in a significant reduction $(p=0.03)$ in $\mathrm{TXB}_{2}$ production in clotted whole blood. The amount of $\mathrm{TXB}_{2}$ generated fell from 1.70 nmoles $/ 10^{9}$ platelets (SEM \pm 0.36 ), to 1.18 nmoles $/ 10^{9}$ platelets $(\mathrm{SEM} \pm 0.22)$.

Despite these changes there were no significant changes in any of the other tests of platelet activity that were performed. There were no changes in platelet aggregation in whole blood measured using the platelet counting technique (Figures 1 and 2). Similarly, the measurements of platelet release reaction using PRP, platelet calcium and magnesium levels, and glycoprotein expression on the membrane surface showed no changes after 6 weeks of fish oil ingestion compared with values obtained prior to the treatment.

Most of the haematological parameters did not change. There was a slight decrease $(p<0.05)$ in the white cell count in whole blood following fish oil ingestion, falling from $6.40 \times 10^{9 / 1}(\mathrm{SEM} \pm 0.25)$ to $5.88 \times 10^{9 / 1}(\mathrm{SEM} \pm 0.40)$. The platelet distribution width decreased slightly $(\mathrm{p}<0.05$ ) from 15.8 fl (SEM $\pm 0.24)$ to $15.1 \mathrm{fl}$ ( SEM \pm 0.09 ). This change was not accompanied by a change in mean platelet volume.

\section{Discussion and conclusions}

Epidemiological data suggest that diets rich in fish oils reduce the incidence of ischaemic heart disease. The beneficial effects of fish oil are thought to be due to the presence of polyunsaturated fatty acids of the $n-3$ family. The fatty acids of the $n-3$ type displace the $n-6$ polyenoic fatty acids, arachidonic acid in particular. Although the physiological significance of changes in prostanoid formation are not clear, changes in the products of fatty acid metabolism, namely the prostaglandins and thromboxanes is thought to play an important role in the regulation of platelet function.

As seen by other investigators (Danders et al 1981, Thorngren and Gustafson 1981, Goodnight et al 1981, Sanders and Roshanai 1983), the ingestion of fish oil significantly changed the platelet fatty acid composition. However these changes in platelet fatty acid profile did not appear to be related to any change 
in platelet behaviour. The reduction in the white cell count we observed has been described in other studies (Hay et al 1982, Mehta et al 1988), but the significance of this observation is unclear. In several studies reviewed by Hornstra (1989) a significant decrease in platelet count was observed, and in other studies an increase in platelet size was observed. In our study the platelet number and size did not change, but a small change in the platelet size distribution was observed which, to our knowledge, has not been observed before and needs to be confirmed by further investigation. The observation of reduced serum $\mathrm{TxB}_{2}$ supports the findings of Knapp et al (1986), and is consistent with the changes in AA content of the platelets.

The results of our study support previous reports where platelet behaviour was not influenced by changes in platelet fatty acids. It is possible that a greater proportion of $20: 5 \mathrm{w} 3$ needs to be incorporated into platelets before changes in platelet behaviour occur. The beneficial effects of fish oil ingestion on the incidence of heart disease may or may not result from an effect on platelet behaviour. It is possible that it is the beneficial changes in cholesterol and reductions in triglycerides (Danders et al 1981, Saynor et al 1984, Singer et al 1983 ) or the reduction of whole blood viscosity (Konayashi et al 1981), that may be causing the reduction in the incidence of thrombosis.

\section{Acknowledgments}

We would like to thank the Haematology Department, University Hospital Nottingham for performing the haematological analysis using the Coulter $S+I V$. Whole blood platelet aggregation was performed by M.E.Burgess-Wilson, J.Crawford, S.Fox, M.Hawkins and J.May. Thromboxane $\mathrm{B}_{2}$ measurements were performed by A.J.Carter and glycoprotein expression was measured by G.I.Johnston. Fatty acid analysis was performed by S.R.Cockbill. We thank also Seven Seas Health Care Ltd. for the gift of MaxEPA and are grateful to the Coronary Artery Disease Research Association for a research grant.

This work was presented at the British Society for Haemostasis and Thrombosis (BSHT), Annual General Meeting, London, 25-26 September 1985 by Cockbill SR, Heptinstall $S$ and Allen BR as part of a paper entitled: The effects of Dietary fish oil supplementation on human blood platelet behaviour in healthy volunteers and in patients with psoriasis. 


\section{References}

Andrews $\mathrm{NP}^{3}$, Broughton Pipkin $\mathrm{F}$ and Heptinstall S. Blood platelet behaviour in mothers and neonates. Thromb Haemostas $1985 ; 53: 428-432$.

Bevan $J$ and Heptinstall $\$$. Serotonin-induced platelet aggregation in whole blood and the eftect of Ketanserin and Mepyramine. Thromb Res 1985; 38:189-194.

Bligh EO and Dyer WJ. A rapid method of total lipid extraction and purification. Canadian Journal of Biochemistry and Physiology 1959:37:911.-917.

Carter A and Hanley SP. The effect of platelet number and haematocrit on whole blood thromboxane synthesis. Thromb Haemostas $1985 ; 53 ; 225-227$.

Cockbill SR, Heptinstall S and Allen BR. The effects of Dietary fish oil supplementation on human blood platelet behaviour in healthy volunteers and in patients with psoriasis. (Abstract). BSHT Annual General Meeting, London, 25-26 September 1985.

Danders TAB, Vickers $M$ and Haines AP. Effect on blood lipids and haemostasis of a supplement of cod-liver oil, rich in eicosapentaenoic and docosahexaenoic acids, in healthy young men. Clinical Science 1981; 61: 317-324.

Dyerberg I and Bang HO. Haemostatic function and platelet polyunsaturated fatty acids in Eskimos. Lancet 1979; ii: pp 433-435.

Glass RL. Alcoholysis, saponification and the preparation of fatty acid methyl esters. Lipids. $1971 ; 6: 919-925$.

Goodnight SH (Jr), Harris SW and Connor WE. The effects of dietary w3 fatty acids on platelet composition and function in man: a prospective, controlled study. Blood 1981; 58 : 880 885 .

Hamberg $N$. ifransformation of $5,8,11,14,17$-eicosapentaenoic acid in human platelets. Biochim Biophy Acta 1980; 618: 389-398.

Hay CRM, Durber AP and Saynor R. Effect of fish oil on platelet kinetics in patients with ischaemic heart disease. Lancet 1982: 1: 1269-1272.

Heptinstall S. The use of a chelating ion-exchange resin to evaluate the effects of a extracelluala calcium concentration on adenosine diphosphate induced aggregation of human blood platelets. Thrombo Haemostas 1976; 36: 208-220.

Hirai A. Hamazaki T, Terano T, et al. Eicosapentaenoic acid and platelet function in Japanese. Lancet 1980; ii: pp 1132-1133. 
Homstra $\mathrm{G}$. The significance of fish and fish-oil coriched food for prevention and therapy of ischaemic cardiowascular disease: VI. Fish (oil) and the function of blood platelets. In. The role of fats in human mutrition 1989. Academic Press Lid, pp. 172-182.

Johnston GI and Heptinstall S. Examination of glycoproteins on intact blood platelets by using monoclonal antibodies and the fuorescence-activated cell sorter. Biochemical Socicty Transactions 1985; 13:109.

Iones EW, Cockbill S, Cowley AJ, Hanley SP and Heptinstall S. Efects of daxoxiben and low dose aspirin on platelet behaviour in man. Br J Pharmacol 1983; 15:39S-44S.

Knapp HR, Reilly IAG, Alessandrini $P$ and FitzGerald GA. In vivo indexes of platelet and vascular function during fish-oil administration in patients with atherosclerosis. New Engl I Med 1986; 314: 937-942.

Kobayashi S, Hirai A, Terano T, et al. Reduction of blood viscosity by eicosapentaenoic acid. Lancet 1981 ; ii: pp 197.

Kronman $\mathrm{N}$ and Green A. Epidemiological studies in the Upernavik district, Greenland. Incidence of some chronic diseases 1950-1974. Acta Medica Scand 1980; 208: 401,-406.

Mehta JL, Lopez LM, Lawson D, Wargovich TJ and Williams LL. Dietary supplementation with stable coronary heart disease. Effects on indices of platelet and neutrophil function and exercise performance. Am J Med 1988; 84: 45-52.

Needleman P, Raz A, Minkes MS, et al. "Triene prostaglandins: prostacyclin and thromboxane biosynthesis and unique biological properties. Proc Nat Aca Sci 1979; 76: 944-948.

Sanders TAB and Roshanai $F$. The influence of different types of w3 polyunsaturated fatty acids on blood lipids and platelet function in heallhy volunteers. Cinical Science 1983; 64: $91-99$

Saynor $R$, Verel $D$ and Gillott $T$. The long-term effect of dietary supplementation with fish lipid concentrate on serum lipids, bleeding time, platelets and angina. Alherosclerosis 1984; 50: $3-10$.

Singer P, Haeger W, Wirth M, et al. Lipid and blood-pressure-lowering elect of mackerel diel in man. Atherosclerosis 1983; 49:99-108.

Thorngmen $M$ and Gustafson A. Effects of 11 -week increase in dictary eicosapentaenoic acid on bleeding time, lipids and platelet aggregation. Lancet 1981; ii: pp 1190-1193. 


\section{CHAPTER 9}

Studies on platelets before and after stroke in a patient with essential thrombocythaemia

Heptinstall S, Cockbill SR, Burgess-Wilson ME and Jones EW. Short report. BMJ 1988, 296: 1298-1299.

\section{Abstract}

An apparently well patient with thrombocythaemia had detailed platelet studies performed 3 days before a stroke and 4 days after. The results compared with normal sex/age matched controls showed the patient to have hyperaggregable platelets before the stroke and hypoaggregable platelets afterwards. 


\section{SHORT REPORT}

Only limited data on the aggregation of platelets before stroke are available; we investigated the behaviour of platelets three days before and four days after acute stroke in a patient with thrombocythaemia.

\section{Case report}

The patient was a 72 year old man who had had a stroke nine months earlier and been prescribed aspirin $300 \mathrm{mg}$ once weekly. Two months later he had developed a mild left hemiparesis and had signs reflecting disorganisation of the aortic valve. A computed tomogram of the head showed diffuse cerebral atrophy but no focal lesion. The platelet count was raised $\left(766 \times 10^{9} / 1\right)$, and results of subsequent laboratory investigations were consistent with essential thrombocythaemia. We concluded that he probably had emboli in the right cerebral hemisphere and prescribed aspirin $1200 \mathrm{mg}$ and dipyridamole $400 \mathrm{mg}$ daily.

He remained well for seven months, when he presented with a focal fit affecting the left arm. Cardiac signs were unchanged, there were no signs of subacute bacterial endocarditis, and cultures of blood yielded negative results. The platelet count was $906 \times 10^{9} / 1$. Aspirin and dipyridamole were withdrawn and. phenytoin sodium $200 \mathrm{mg}$ nightly was started. The focal epileptic fit was thought to have been related to cortical scarring after his previous strokes.

Thirteen days later he returned as an outpatient, feeling well and having had no further neurological symptoms. His platelet count was $852 \times 109 /$, and detailed platelet studies were performed. Three days later he was re-admitted to hospital having collapsed and developed a right hemiplegia. Initially he seemed to recover, and four days after admission blood was taken for platelet studies; the platelet count had fallen to $557 \times 10 \% /$. His condition deteriorated rapidly shortly afterwards, and he died a day later. Consent for a necropsy was refused.

Platelet studies - Aggregation of platelets was measured in stirred citrated whole blood and heparinised whole blood with a platelet counting technique (1). Results were compared with those for 20 apparently healthy men (mean age 71, range 61-83). The figure shows that the patient's platelets were hyperaggregable before the stroke and hypoaggregable afterwards. Resistance of aggregation to epoprostenol was assessed in plasma rich in platelets prepared from heparinised blood with $1 \mathrm{mmol} / \mathrm{L}$ sodium arachidonate as the aggregating 
agent (2). The results were compared with those for 14 apparently healthy men (mean age 70, range 61-83). A concentration of $10 \mathrm{mmol} / \mathrm{l}$ epoprostenol was sufficient to prevent completely the aggregation of platelets (and the release reaction that accompanies it) in plasma from the controls; in contrast, $25 \mathrm{nmol} / 1$, the highest concentration of epoprostenol used, did not affect aggregation of platelets in plasma taken from the patient either before or after his stroke.
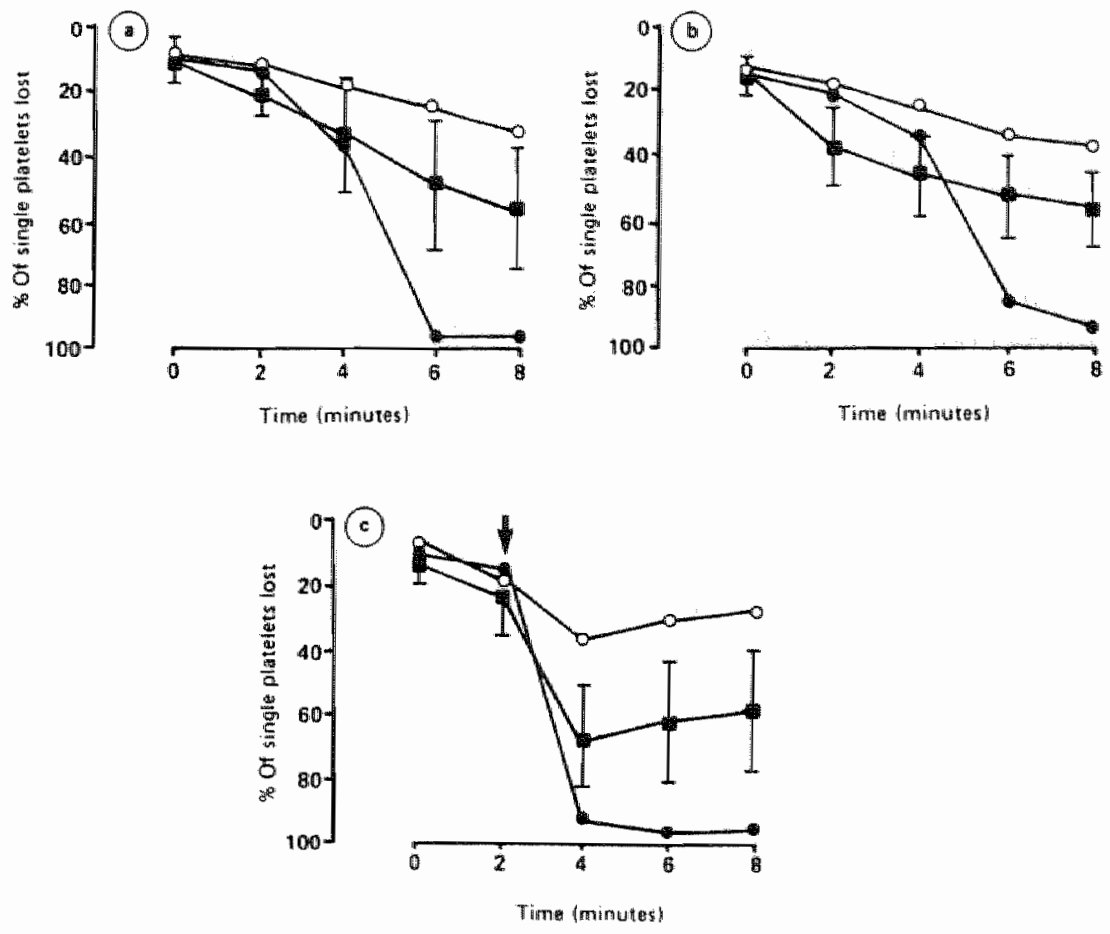

\section{Figure 1}

Fig. 1 Platelet aggregation (percentage of single platelets lost) occurring in (a) stirred citrated blood; (b) stirred heparinised blood; and (c) citrated blood to which ADP $(0.5 \mu \mathrm{mol} / 1)$ wass added (arrowed). (-) patient before stroke; $(O)$ patient after stroke; ( normal controls, verticals bars represent the standard deviation. 


\section{Comment}

There have been many studies of aggregation of platelets after stroke (3), but little data exists on aggregation immediately before stroke. The patient reported on here had hyperaggregable platelets before the stroke and hypoaggregable platelets (and a lower platelet count) after it. The hyperaggregable platelets may possibly have participated in the formation of thrombus and not been available in the circulation after the stroke; alternatively, natural protective mechanisms may have operated to reduce the aggregability of platelets.

Although aggregation of platelets was reduced after the stroke, it continued to be resistant to the inhibitory effects of epoprostenol. Resistance to inhibitory prostaglandins in thrombocythaemia has recently been reported by others (4). That this abnormality was present both before and after stroke in our patient suggests that they are not major determinants of the extent of aggregation of platelets in whole blood. Nevertheless, the resistance of aggregation to inhibition by epoprostenol may be relevant in vivo.

Preston et al found that patients with high platelet counts and neurological symptoms improved considerably with treatment with aspirin and dipyridamole (5). The fact that in our patient the acute stroke occurred only 16 days after treatment was withdrawn may be important; aspirin and dipyridamole may have been providing protection by reducing the aggregability of platelets.

\section{References}

1. Fox SC, Burgess-Wilson M, Heptirnstall S and Mitchell JRA. Platelet aggregation in whole blood determined using the Utra-Flo 100 platelet counter. Thromb Haemostas $1982 ; 48: 327-329$.

2. Andrews NP. Bronghton Pipkin F, and Heptinstall S. Blood platelet behaviour in mothers and neonates. Thromb Haemost 1985:53:428-32.

3. Heptinstall S and Mitchell JRA. Platelets and thrombosis. In; Human blood coagulation, haemostasis and thrombosis. 3rd ed. Biggs R and Rizza CR, eds. Blackwell, Oxford, $1984 ; p p 380-414$

4. Contelazzo S. Castagna D, Galli M, Barbui T and de Gaetano G. Increased response to arachidonic acid and $\mathrm{U}-46619$ and resistance to inhibitory prostaglandins in patients with chronic myeloproliferative disorders (Abstract). Thromb Haemostas 1987; 58:159.

5. Preston FE, Martin JF, Stewart RM and Davies-Jones GAB. Thrombocytosis, circulating platelet aggregates, and neurological dysfunction. $\mathrm{Br} \mathrm{Med} .11979$; ii, pp $1561 \cdots 1563$ 


\section{The study of whole blood aggregation in a group of patients after suspected myocardial infarction}

\section{Abstract}

The objectives of this study were to investigate whether changes in platelet reactivity could be detected in patients after an acute thrombotic event such as a myocardial infarction. It was hoped to observe any subsequent changes in platelet behaviour and relate them to further thrombosis and mortality. Whole blood platelet aggregation (WBA) and agonist-induced serotonin (5HT) release from platelet-rich plasma were measured in 80 individuals with suspected myocardial infarction (MI) within 1 day, 1 week and 1 month after the event. In the first 24 hours after the event there was no difference in platelet activity seen in patients who had had an MI and the normal control group. No relationships were seen between platelet activity and the extent of the MI or the development of further thrombosis. Increased platelet activity was seen in untreated patients 1 week after the MI but not in patients given the thromboxane synthetase inhibitor Dazoxiben. The group of untreated patients also showed, after I week, a correlation between the cardiac enzyme levels and both the TxB $\mathrm{B}_{2}$ synthesis in whole blood and the platelet $5 \mathrm{HT}$ release, suggesting a relationship with the extent of cardiac damage sustained. This relationship was not seen in the group treated with Dazoxiben. Dazoxiben inhibited both the $T \times B_{2}$ synthesis in whole blood and the release of agonist-induced $5 \mathrm{HT}$ from PRP, but had no effect on either WBA or the incidence of subsequent venous thrombosis. The lack of effect of Dazoxiben on WBA suggested that WBA is not dependent on thromboxane synthesis. Increased platelet activity was measured within 1 day of the MI in the patients who died within 4 weeks of the event, compared with the patients who did not die. The extent of this phenomenon was, however, not sufficiently large to use these measurements of platelet activity to identify patients with a poor prognosis. 


\section{Introduction}

There have been many reports that suggest that myocardial infarction (MI) is associated with platelet hyperactivity (Grant and Prentice 1994). It is thought that this hyperactivity may predispose such patients to develop further thrombi in the form of deep vein thrombosis (DVT). Many investigators have studied platelets collected from patients post MI in an attempt to develop tests of platelet behaviour that might predict the development of DVT, but such tests have proved elusive (Hirsh 1981, Heptinstall and Mitchell 1984). In such studies where the investigation was carried out after the $M I$ it is difficult to determine whether any changes in platelet activity are due directly to the $\mathrm{MI}$ or have resulted from it. If thrombi have been formed in vivo it is possible that a hyperactive subpopulation of platelets may have already been lost from the circulation into the thrombus. This would leave normal or even relatively inactive platelets in the blood samples examined. It is also possible that the tissue damage associated with the MI may release substances that might make circulating platelets hyperactive. One method of resolving these questions might be to measure platelet activity sequentially after the MI and see if the platelet reactivity changes, and whether these changes can be related to the severity of the MI or the development of complications such as DVT.

This study was designed to investigate platelet activity changes by measuring platelet aggregation in whole blood and the release of platelet serotonin (5HT) from platelet-rich plasma (PRP). A group of patients with suspected MI were investigated within 24 hours of hospital admission before randomisation to receive either placebo or a thromboxane synthetase inhibitor (Dazoxiben). The effect of the ingestion of an anti-platelet drug Dazoxiben on the formation of DVT and platelet activity was also investigated by testing the surviving patients at one week and one month after hospital admission. "The results obtained within 24 hours of admission were related to patient survival after one month in an attempt to assess the measurement of platelet hyperactivity as an indicator of poor prognosis (Heptinstall and Mulley 1977). A group of normal, age and sex matched individuals were used as the control group.

\section{Materials}

The heparin used was bactericide-free heparin injection B.P. (Monoparin) from Weddel Pharmaceuticals Limited, supplied at a concentration of 5000 units $/ \mathrm{ml}$ (stock solution). The solution that was used to anticoagulate blood was a 1 in 10 dilution of the stock solution in saline. A $20 \mu$ l aliquot was added to a plastic 
tube $(10 \mathrm{ml}$ capacity) with $180 \mu$ saline solution, to which $10 \mathrm{ml}$ of whole blood was added. The solution of citrate that was used to anticoagulate blood was $3.8 \%(w / v)$ trisodium citrate dihydrate. A $1 \mathrm{ml}$ aliquot was added to a plastic tube, to which $10 \mathrm{ml}$ of whole blood was added. Tubes $(4 \mathrm{ml}$ capacity) that contained $6.4 \mathrm{mg}$ of potassium EDTA were obtained from Labco Limited. Adenosine 5'-diphosphate (ADP, sodium salt) and adrenaline (ADR) were obtained from Sigma Chemical (St. Louis). The ADP and ADR were dissolved and diluted in saline before use. The $0.9 \%(\mathrm{w} / \mathrm{v}) \mathrm{NaCl}$ used was prepared by the Boots Chemical Co. for infusion purposes. The arachidonic acid (AA, grade 1 from porcine liver) was obtained from Sigma Chemical (St. Louis), and was converted to the sodium salt before use by dissolving in $0.1 \mathrm{~mol} / \mathrm{l}$ of sodium carbonate. The $\left[{ }^{14} \mathrm{C}\right]$-serotonin $(5 \mathrm{HT})$ were obtained from Amersham International, Amersham, Bucks, UK. The anti-Thromboxane $\mathrm{B}_{2}$ antibody was a gift to Dr. A. J. Carter from Professor Levine, Brandies University, USA.

\section{Methods}

PRP release reaction. Platelet-rich plasma (PRP) was prepared from heparinised blood and the release of $[14 \mathrm{C}]$-serotonin (5HT) was measured as described previously (Heptinstall and Mulley 1977). Aggregation was stimulated using the following platelet agonists; ADP, ADR and AA. The arachidonic acid threshold (AA Thres) was defined as the lowest concentration (in $\mathrm{mM}$ ) of arachidonic acid that induced SHT release from the platelets.

Whole blood platelet aggregation. Citrated and heparinised whole blood were tested for 'spontaneous' platelet aggregation (SPA) using the method previously described (Fox et al 1982). The effect of adding ADP in a final concentration of $0.5 \mu \mathrm{M}$, to citrated blood was also measured. The ADP was added after 2 minutes stirring, and then the sample was stirred for a further 6 minutes. Platelet counts were measured after $0,2,4,6$ and 8 minutes of stirring.

Thromboxane $B_{2}$ measurement $\left(T x B_{2}\right)$. This was measured in a clotted whole blood sample by the method described by Carter et al 1984 .

${ }^{125}$ I-Fibrinogen leg scans. 125 I-fibrinogen leg scans were performed as previously described (Morris and Mitchell 1977). This test was performed within the first week of hospitalisation. 
Hatmatological paramerers. An erythrocyte sedimentation rate (ESR) estimation and a full blood count using the Coulter S+IV were performed by the Department of Haematology, University Hospital Nottingham.

Clinical chemistry parameters. The cardiac enzymes aspartate amino transferase (AST) and hydroxybutyrate dehydrogenase (HBD) were measured during the first three days of hospitalisation by the Clinical Chemistry Department, University Hospital Nottingham. These enzyme levels were used to dentify the existence of an MI and measure its severity using the criteria described by Wilcox et al 1981.

\section{Sample collection and medication}

On each test occasion the following blood samples were taken; 4mI EDTA sample, 20ml heparinised sample for platelet-rich plasma (PRP) preparation, $10 \mathrm{~m} /$ heparinised sample and $10 \mathrm{ml}$ citrated sample for whole blood aggregation (WBA) studies, $5 \mathrm{ml}$ clotted sample for $\mathrm{TxB}_{2}$ determination and another $5 \mathrm{ml}$ clotted sample for cardiac enzyme determination. Three samples were collected from each patient, whenever possible. The first sample (Day 1) was taken on the first morning after the patient's admission into hospital with a suspected MI. This sample was taken before randomisation onto either Dazoxiben or placebo capsules. A second sample (1 week) was taken $\mathbb{1}$ week after hospital admission, and a third sample ( 1 month) was taken 1 month after hospital admission. The Dazoxiben or placebo capsules were taken from after the Day 1 sample had been collected until one month after hospital admission.

\section{Patients and control subjects}

Patients. All the patients were over 45 years old and denied regular ingestion of any drugs likely to affect platelet behaviour and were not being treated with any anticoagulant drugs. Within the study period these drugs were prohibited unless they were considered essential by the clinicians in charge of the patient. Of the 90 patients tested on Day 1, 80 developed changes in the electrocardiogram (ECG) and/or levels of cardiac enzymes that suggested a recent MI. These were classified according to the criteria of Wilcox et al 1981 as: 35 with definite MI, 21 with probable MI and 24 with possible infarctions. The other 10 individuals all had previous histories of ischaemic heart disease (IHD). 
Control subjects. There were 32 age and sex matched individuals studied, all were over 46 years old and denied serious illness and any medication in the two weeks prior to the blood collection.

\section{Results}

Of the 80 patients with a suspected MI (MI patients) investigated on Day 1, 78 were investigated again at 1 week ( 42 were taking placebo and 36 were taking Dazoxiben) and 70 were investigated at 1 month (37 were taking placebo and 33 were taking Dazoxiben).

\section{Whole blood platelet aggregation (WBA)}

The mean extent of WBA seen in the different groups tested was remarkably similar overall. Figure 1 shows the mean results from all the MI patients $(n=80)$ compared with the control group $(\mathrm{n}=32)$. The results obtained in patients with definite MI, probable MI, possible MI were the same as those obtained from the control group (data not shown). The results obtained after 1 week in MI patients taking placebo capsules were no different from the results obtained on Day 1 for all MI patients (Figure 2), and the results obtained from MI patients who had taken Dazoxiben for 1 week were the same as those Ml patients taking placebo (Figure 3). There were no relationships between the extent of WBA and size of the infarct as judged by the level of cardiac enzymes. The extent of WBA on Day 1 was not different in MI patients that that either did or did not develop positive 125 I-Fibrinogen leg scans, an indication of a DVT, (Figure 4) and the results obtained at Day 1 and after 1 week from patients that had developed positive leg scans were similar (data not shown).

The only significant difference seen in the extent of WBA was an increase measured at Day 1 in patients that died within 4 weeks of admission compared with patients that survived for 4 weeks (Figure 5). Although the patients that died were on average older than those that survived (Mean $\pm S E M, 71 \pm 1.5$ and $66 \pm 0.9$ respectively) a comparison of two groups of normal subjects with a comparable age difference (Mean $\pm S E M, 71 \pm 0.4$ and $64 \pm 0.4$ respectively), showed no significant differences in whole blood platelet aggregation when either citrated or heparinised blood was used as anticoagulant (data not shown). 
Perconlage plateletest remalining in $\mathrm{CB}$
Percentage gidalelets. reirialning in $\mathrm{CB}$
Percentage platelets

remalning in $H B$

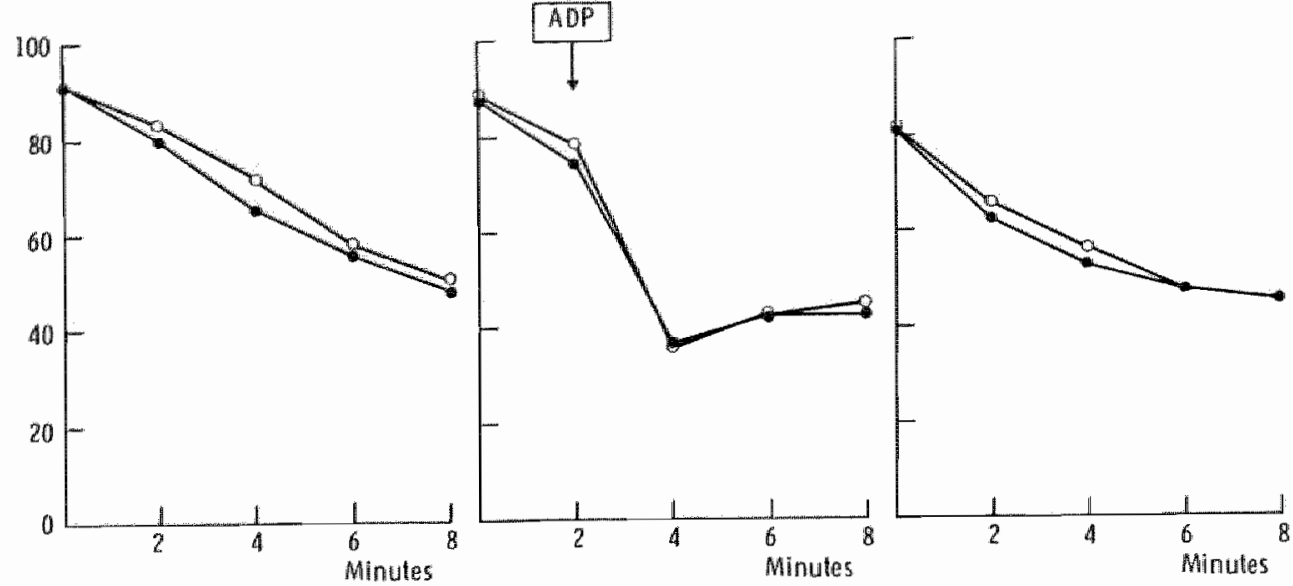

Figure 1

Fig. I The results show the mean values of the extent of WBA on Day I in all patients with enzyme and ECG changes suggesting myocardial infarction (MI), $\mathrm{n}=80(\mathrm{O})$, and healthy controls, $\mathrm{n}=32$ (). $\mathrm{CB}=$ citrated blood, $\mathrm{HB}=$ Heparinised blood. The $\mathrm{ADP}$ (final concentration $0.54 \mathrm{M}$ ) was added after 2 minutes of stiming.

\section{Percentage platehets remalning in $\mathrm{CB}$}

\section{Percentage platelets remaining in $\mathrm{CB}$}

\section{Percentage platelets remaining in $\mathrm{HB}$}

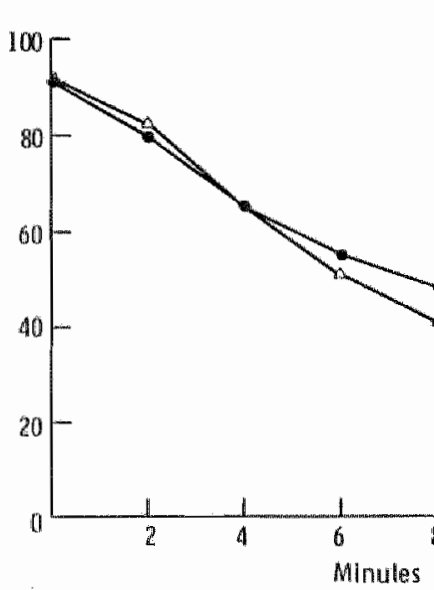

\section{Figure 2}
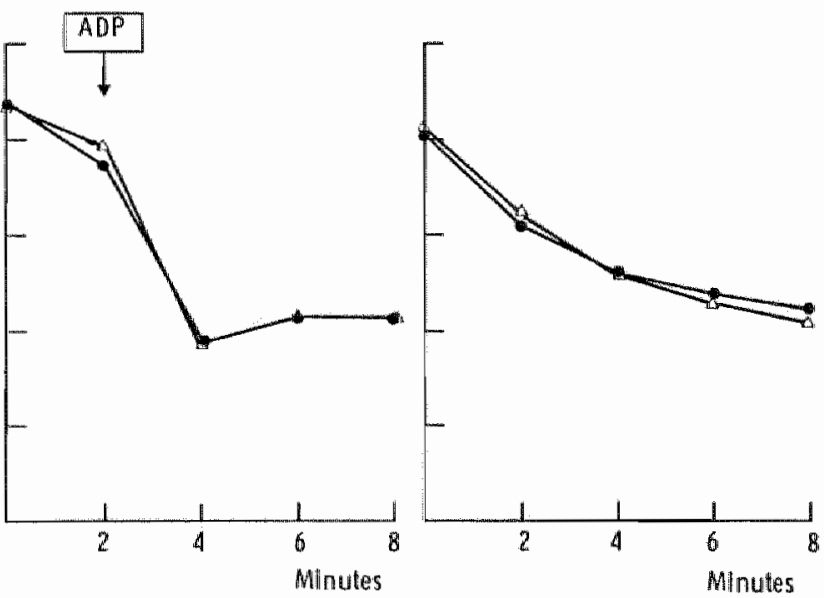

Fig. 2 The results show the mean values of WBA on Day 1 in all patients with enzyme and ECG changes suggesting myocardial infarction (MI), $n=80(-)$, and the results in patients after 1 week of receiving the placebo capsules, $\mathrm{n}=42(\Delta) . \mathrm{CB}=$ citrated blood. $\mathrm{HB}=$ Heparinised blood. The ADP (final concentration $0.5 \mu \mathrm{M}$ ) was added after 2 minutes of stirring. 


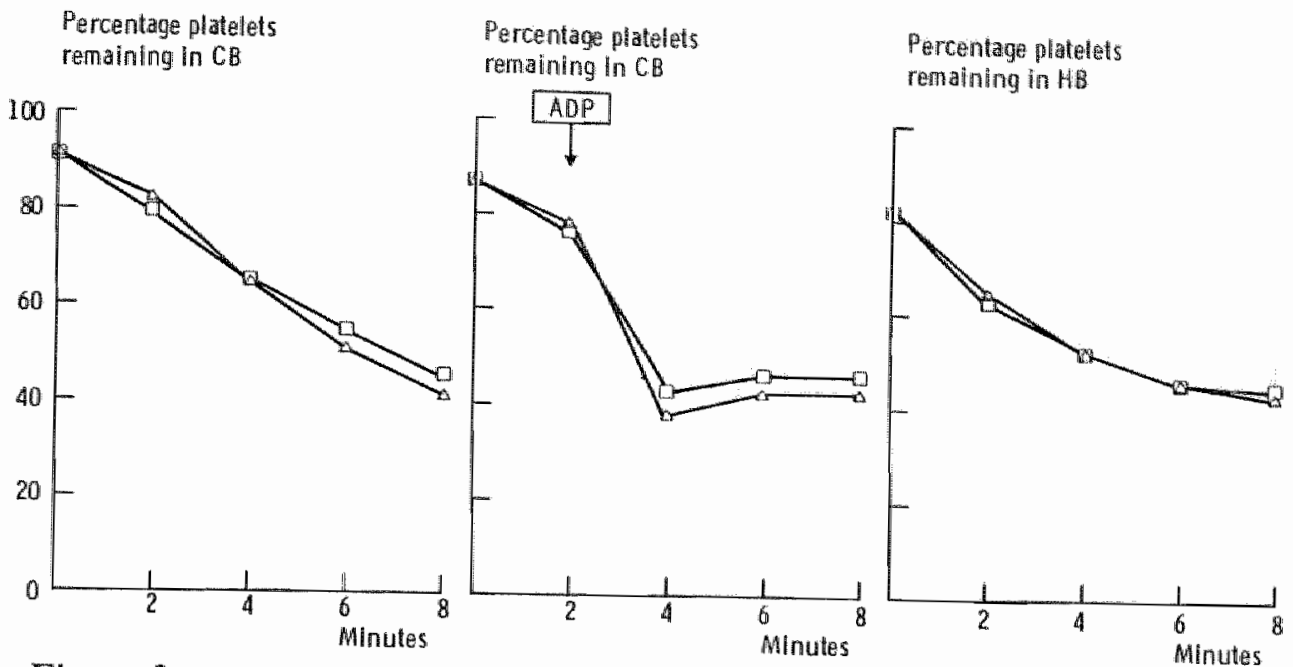

\section{Figure 3}

Fig. 3 The results show the mean values of the extent of WBA in patients at 1 week taking Dazoxiben, $n=36(\square)$ and placebo capsules, $n=42(\Delta) . C B=$ citrated blood, $\mathrm{HB}=$ Heparinised blood. The $\mathrm{ADP}$ (final concentration $0.5 \mu \mathrm{M}$ ) was added after 2 minutes of stirring.

Percentage platelets

remaining in $C B$

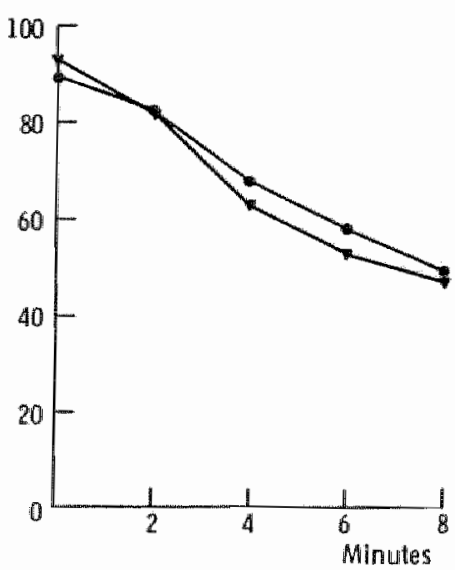

Percentage platelets. remaining in $\mathrm{CB}$

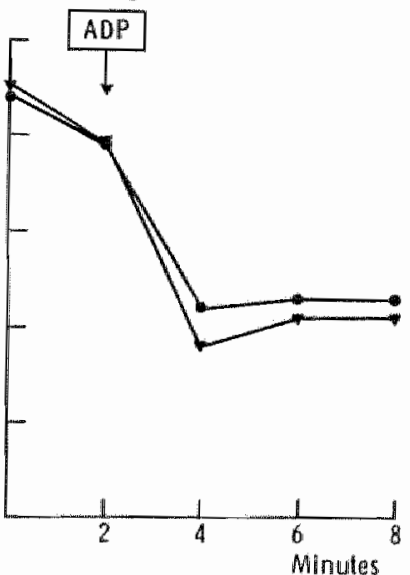

\section{Percentage platelels remaining in $H B$}

Figure 4

Fig. 4 The results show the mean values of the extent of WBA in patients on day 1 who subsequently developed positive leg scans, $n=17(\nabla)$ and in those whose scans remained negative, $\mathrm{n}=73, \mathrm{CB}=$ citrated blood, $\mathrm{HB}=$ Heparinised blood. The $\mathrm{ADP}$ (final concentration $0.5 \mu \mathrm{M}$ ) was added after 2 minutes of stirring. 
Percontaga pallelets

remalning in $\mathrm{cos}$
Percontage platelats renatning hin $\mathrm{C}$
Percentuge platelets remathing in $\mathrm{HB}$

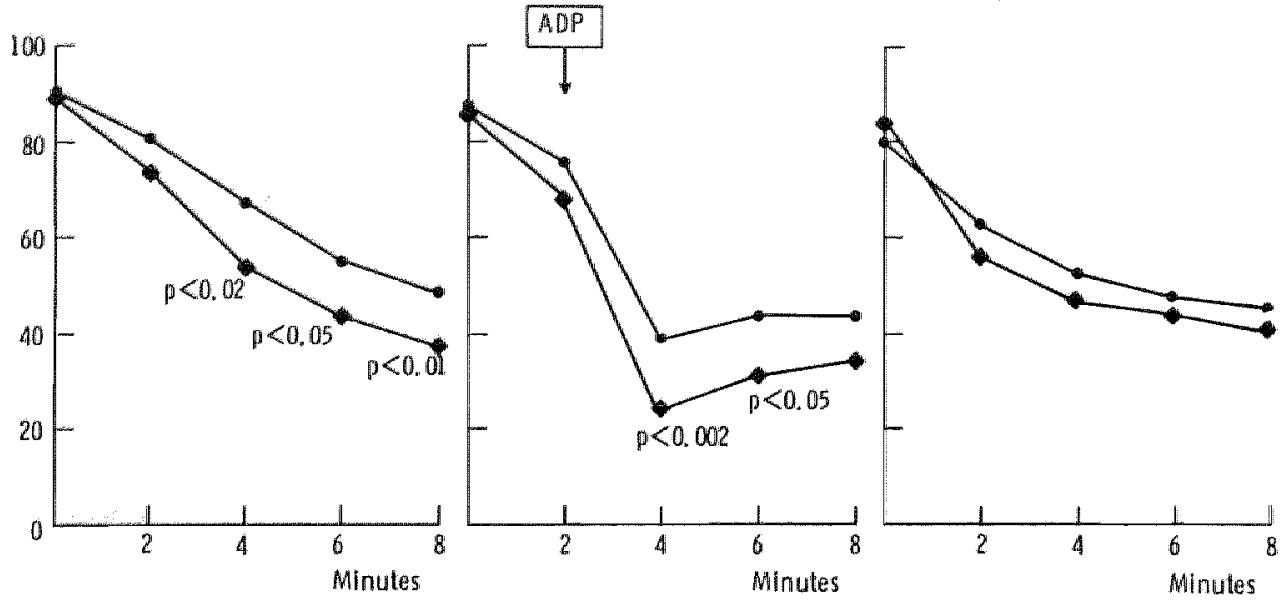

Figure 5

Fig. 5 The results show the mean values of the extent of WBA in patients on day 1 who subsequently died within 4 weeks, $n=14$ ( ) and in those that survived for 4 weeks, $n=76$ ( $\mathrm{CB}=$ citrated blood $\mathrm{HB}=$ Heparinised blood. "The $\mathrm{ADP}$ (final concentration $0.5 \mu \mathrm{M}$ ) was added after 2 minutes of stirring.

\section{Platelet studies using platelet-rich plasma (PRP)}

The results obtained within 24 hours of admission (Day 1) from MI patients and controls (Table 1) were not significantly difference. There were no relationships between the size of the infarct as judged by the cardiac enzyme levels and the release of platelet 5 HT (results not shown). In contrast with the WBA results, the platelet 5HT release was increased after 1 week from the MI patients on placebo (Table 3) compared with the results obtained from all the MI patients on Day 1, with those on placebo at 1 month (Table 3) and with the controls (Table 1). In the subgroup with a definite $\mathrm{MI}$ the increased 5HT release at 1 week was particularly striking (Table 2). The results obtained from the MI patients 1 month after admission who were taking placebo (Table 3) were not significantly different to either the results obtained on Day 1, or the control group (Table 1). 
Table 1 Results at Day 1 compared to control groups - Mean (SEM)

\begin{tabular}{|c|c|c|c|c|c|}
\hline \multirow[t]{2}{*}{ Parameter } & \multicolumn{3}{|c|}{ MI group - Day 1} & \multirow[t]{2}{*}{ IHD } & \multirow[t]{2}{*}{ Controls } \\
\hline & Definite & Probable & Possible & & \\
\hline AA Thres & $0.49(0.04)$ & $0.42(0.05)$ & $0.48(0.04)$ & $0.51(0.06)$ & $0.56(0.05)$ \\
\hline $\mathrm{ADP} 10 \mu \mathrm{M}$ & $\overline{14(2.1)}$ & $13(2.9)$ & $16(3.0)$ & $10(2.8)$ & $12(2.1)$ \\
\hline ADR $10 \mu M$ & $18(3.2)$ & $15(3.3)$ & $22(4.2)$ & $15(5.6)$ & $16(3.0)$ \\
\hline ADR $100 \mu \mathrm{M}$ & $24(3.3)$ & $21(3.8)$ & $27(4.2)$ & $17(5.5)$ & $21(3.2)$ \\
\hline Mean age & 67.0 & 67.1 & 67.9 & 62.7 & 63.7 \\
\hline $\mathrm{n}$ & $\overline{35}$ & 21 & 24 & 10 & 32 \\
\hline
\end{tabular}

Table 2 PRP results in patients with definite MI - Mean (SEM)

\begin{tabular}{|c|c|c|c|}
\hline Parameter & Day 1 & $\begin{array}{c}1 \text { week } \\
\text { (on placebo) }\end{array}$ & $\mathrm{p}<$ \\
\hline AA Thres & $0.49(0.04)$ & $0.31(0.03)$ & 0.001 \\
\hline ADP $10 \mu \mathrm{M}$ & $14.0(2.1)$ & $30.1(5.0)$ & 0.01 \\
\hline ADR $10 \mu \mathrm{M}$ & $17.6(3.2)$ & $33.8(6.3)$ & 0.02 \\
\hline ADR $100 \mu \mathrm{M}$ & $23.7(3.3)$ & $41.6(5.4)$ & 0.01 \\
\hline
\end{tabular}

Table 3 Comparison of patients taking placebo or Dazoxiben - Mean (SEM)

\begin{tabular}{|c|c|c|c|c|}
\hline \multirow[t]{2}{*}{ Parameter } & \multicolumn{2}{|c|}{ Placebo } & \multicolumn{2}{|c|}{ Dazoxiben } \\
\hline & 1 week & 1 month & 1 week & 1 month \\
\hline AA Thres & $0.39(0.02)$ & $0.48(0.04)$ & $0.42(0.03)$ & $0.49(0.04)$ \\
\hline $\mathrm{ADP} 10 \mu \mathrm{M}$ & $20(2.6)^{*}$ & $15 \overline{(2.7)^{*}}$ & $11(1.7)$ & $9(1.5)$ \\
\hline ADR $10 \mu \mathrm{M}$ & $24(3.4)^{*}$ & $20(3.6)$ & $15(2.5)$ & $15(2.6)$ \\
\hline ADR $1004 M$ & $31(3.3)^{*}$ & $22(3.6)$ & $23(2.9)$ & $19(2.6)$ \\
\hline
\end{tabular}

* Significantly different from Dazoxiben group $(p<0.05)$

The MI patients tested 1 week after admission who were taking Dazoxiben had significantly reduced platelet $5 \mathrm{HT}$ release induced by ADP and adrenaline (Table 3) compared with those taking placebo. In the same groups of $\mathrm{Ml}$ 
patients tested 1 month after admission only the platelet 5 HT release induced by $104 M$ ADP was significantly lower. There was a correlation of the amount of released platelet SHT (induced by $10 \mu \mathrm{M}$ ADP measured at 1 week) in the MI patients on placebo with the peak levels of the cardiac enzymes AST and HBD $(r=0.5, p<0.05$ and $r=0.55, p<0.01$ respectively). The results from $\mathrm{MI}$ patients taking Dazoxiben did not show this correlation and had a simillar extent of release to that of $\mathrm{MI}$ patients on Day 1 and the control group. There were no significant differences in the extent of platelet $5 \mathrm{HT}$ release seen in those who did and did not develop positive leg scans, i.e. those who did or did not develop a DVT (data not shown).

There was, however, some evidence of greater platelet activity in those patients that died within 4 weeks compared with controls ( $p<0.05$ ) (Table 4) and the results were significantly different from those who did not die within 4 weeks.

\section{Table 4 Comparison of patients who died within one month compared with those who survived longer than 1 month - Mean (SEM)}

\begin{tabular}{|c|c|c|}
\hline \multirow{2}{*}{ Parameter } & \multicolumn{2}{|c|}{ Results on Day 1 } \\
\cline { 2 - 3 } & Died & Survived \\
\hline AA Thres & $0.43(0.04)^{*}$ & $0.48(0.02)$ \\
\hline ADP $10 \mu \mathrm{M}$ & $20(4.6)^{*}$ & $13(1.3)$ \\
\hline ADR $10 \mu \mathrm{M}$ & $19(5.2)$ & $17(2.1)$ \\
\hline ADR $100 \mu \mathrm{M}$ & $28(5.6)$ & $22(2.2)$ \\
\hline
\end{tabular}

* Significantly different from survivors $(p<0.05)$

\section{Thromboxane $B_{2}\left(T x B_{2}\right)$ levels}

The levels of $\mathrm{TxB}_{2}$ synthesis in clotted whole blood measured on Day 1 were the same in MI patients and the control group and the $T \mathrm{~TB}_{2}$ level did not correlate with the size of infarct as judged by the cardiac enzyme levels (data not shown). The $\mathrm{TxB}_{2}$ levels were not different in patients with and without positive leg scans, i.e. with or without a DVT, or in patients who survived for, or died within 4 weeks (data not shown). As expected the MI patients taking the thromboxane synthetase inhibitor Dazoxiben had reduced $\mathrm{TXB}_{2}$ levels at 1 week and 1 month. Interestingly, in the group of MI patients taking placebo a strong correlation $(r=0.8)$ was seen between the $\mathrm{TxB}_{2}$ levels and cardiac enzyme levels (AST and $\mathrm{HBD}$ ) at 1 week which still persisted at 1 month, suggesting that the 
$T \times B_{2}$ synthesis in clotted whole blood may relate to the extent of cardiac damage sustained.

\section{Haematological parameters}

None of the haematological parameters measured correlated with platelet activity, cardiac enzyme levels or the occurrence of DVT as identified by leg scans.

\section{Discussion and conclusions}

The objectives of this study were to investigate whether changes in platelet reactivity using either whole blood aggregation (WBA) of platelet serotonin (5HT) release from platelet-rich plasma (PRP) could be detected in the patients after an acute thrombotic event such as myocardial infarction (MI). It was hoped to observe any subsequent changes in platelet behaviour and relate them to the development of further thrombosis (DVT) or to mortality. WBA was compared with the agonist-induced platelet 5HT release from PRP, and the action of the antiplatelet drug, Dazoxiben, on both these measurements of platelet behaviour was investigated. The extent of WBA seen in the different groups tested was remarkably similar overall. However, there were several changes in $\mathrm{TxB}_{2}$ levels and platelet reactivity in PRP when no change in WBA was seen.

When the MI patients were tested within 24 hours of admission (Day 1) the extent of platelet activity (measured in whole blood or PRP) and the $\mathrm{T}_{\mathrm{B}} \mathrm{B}_{2}$ levels were identical to those of the control group. The observation that the platelet activity present within the 24 hour period after an MI is not different from normal individuals supports the observations of investigators (Heptinstall et al 1980) who demonstrated that the extent of released $5 \mathrm{HT}$ from platelets (PRP) using ADP as an agonist was not different in patients who had suffered an $\mathrm{MI}$ and normal controls. The platelet reactivity in PRP and $\mathrm{TxB}_{2}$ levels measured in $\mathrm{MI}$ patients on placebo (especially those with definite $\mathrm{Ml}$ ) were increased at 1 week compared to the results obtained on Day 1, whilst no difference was seen in WBA results. These increases had disappeared when patients were tested at 1 month, suggesting a transient increase in platelet activity due to the MI. 
None of the measurements of platelet reactivity (in whole blood or PRP) were able to predict further thrombosis in the form of DVT, as detected by radiolabelled fibrinogen leg scans, although other investigators have noted that the risk of developing DVT after MI may be related to the severity of the infarct (Hayes et al 1976). The size of the infarct can be judged from the cardiac enzyme levels (Wilcox et al 1981). Within the 24 hour period after the MIt there was no correlation between the $\mathrm{TxB}_{2}$ levels and the size of the infarct. However after 1 week, in the group of MI patients taking placebo the cardiac enzyme levels showed a correlation with $T \times B_{2}$ synthesis and platelet $5 H T$ release, which suggests a relationship with the extent of cardiac damage sustained. This correlation was not seen in the group taking Dazoxiben. The antiplatelet drug Dazoxiben was shown to effectively inhibit the platelet production of $T \times B_{2}$ at $]$ week and 1 month as expected, and it reduced the release of agonist-induced 5HT from PRP. Dazoxiben had no effect, however, on either the incidence of subsequent venous thrombosis or the extent of WBA measured. As the thromboxane synthetase inhibitor reduced platelet granule content release but had no effect on the platelet aggregation in whole blood, it seems possible that WBA is not dependent on thromboxane synthesis.

The results using either whole blood and PRP showed that platelet activity was increased in those individuals who died within 4 weeks of the thrombotic event compared with those that survived, even taking into account the small difference in the mean ages of the two groups $(71$ years and 66 years, respectively). There was no evidence of an increase in the incidence of DVT post $\mathrm{MI}$ in this study, and although the changes in platelet activity were statistically significant, the differences was not large enough for it to be used to identify beforehand individuals with a poor prognosis. However, the observation of increased platelet reactivity supports the results of investigators (Heptinstall et al 1980, Trip at al 1990) who have showed a significant relationship between the extent of ADP-induced platelet release reaction and the prognosis after an $\mathrm{MI}$, and other studies that have demonstrated an association between platelet aggregation and MI (Elwood et al 1991, Thaulow et al 1991).

The results from this study suggest that there is a transient change in platelet reactivity after the acute thrombotic event of MI. However, as the results obtained within 24 hours of the event were similar to normal controls any subsequent changes probably are related to the damage caused by the MI. Although it is possible that same mechanism that leads to the increased platelet reactivity after an acute thrombotic event also increases the overall risk of thrombosis, it will be impossible to separate cause from effect without prospective studies. 


\section{Acknowledgments}

The platelet $5 \mathrm{HT}$ release reaction testing was performed by $\mathrm{I}$. Crawford and $\mathrm{M}$. Hawkins. The $T \times B_{2}$ testing was performed by $A$. Carter. The Haematology Department, University Hospital Nottingham performed the full blood counts and ESR determinations and the Clinical Chemistry Department, University Hospital Nottingham performed the cardiac enzyme testing. E. Holland performed the leg scans. Dr. E. W. Jones organised the study and selection of the patients, took the blood samples and performed the clinical assessments. Dr. E. W. Jones used data from this study as part of his thesis for the degree of Doctor of Medicine; Venous thromboembolism : Studies of aspects of its pathogenesis and treatment, submitted to the University of Nottingham for the degree of Doctor of Medicine, August 1986.

\section{References}

Carter AJ, Bevan JA, Hanley SP, Morgan WE and Turner DRA. A comparison of human pulmonary arterial and venous prostacyclin and thromboxane production - effect of a thromboxane synthetase inhibitor. Thromb Haemost 1984; 51:257-260.

Elwood PC, Renaud S, Sharp DS, Beswick AD, O'Brien JR and Yarnell JWG. Ischaemic heart disaese and platelet aggregation: The Caerphilly Collaborative Heart Disease Study. Circulation 1991; 83: 38-44.

Fox SC, Burgess-Wilson M, Ileptinstall S. Mitchell JRA. Platelet aggregation in whole blood determined using the Ultra-Flo 100 whole blood platelet counter. Thromb Hacmost 1982; 48 : $327-329$.

Grant PJ and Prentice CRM. Coronary artery disease. In: Haemostasis and thrombosis. Bloom AL, Forbes CD. Thomas DP and Tuddenham EGD, eds. Churchill Livingstone, New York. 1994: pp 1231-1254.

Hayes MJ, Morris GK and Hampton JR. Lack of effect of bed rest and cigarette smoking on development of deep venous thrombosis after myocardial infarction. Br Heart I 1976; 38: $981-983$.

Heptinstall S and Mitchell JRA. Platelets and thrombosis. In: Biggs R and Rizza CR (eds), Human blood coagulation, haemostasis and thrombosis. (3rd Edition). Oxford. Blackwell Scientific Publications 1984; pp 380-415.

Heptinstall S, Mulley GP. Taylor PM and Mitchell JRA. Platelet release reaction in myocardial infarction. Br Med J 1980; 1:80-81. 
Heptinstall S and Mulley GP. Adenosine diphosphate induced platelet aggregation and release reaction in heparinised platelet rich plasma and the influence of adding citrate $\mathrm{Br} J$ Haematol $1977 ; 36: 565-571$.

Hirh I. Blood tests for the diagnosis of venous and arterial thrombosis. Blood 1981; 57 : 1-8.

Morris GK and Mitchell JRA. Evaluation of 125I-fibrinogen test for venous thrombosis in patients with hip fractures: comparison between isotope scanning and necropsy finding. $\mathrm{Br}$ Med J 1977: 1: 264-266.

Thaulow E, Erikssen J, Sandvik L, Stormorken H and Cohn PF. Blood platelet count and function are related to total and cardiovascular death in appartently healthy men. Circulation $1991 ; 84: 613-617$

Trip MD, Cats VM, van Capelle FJL and Vreeken J. Platelet hyper-reactivity and prognosis in survivors of myocardial infarction. New Engl J Med 1990; 322: 1549-1554.

Wilcox RG, Roland $\mathbb{J}$ and Hampton JR. Prognosis of patients with "Chest Pain? cause". Br Med J 1981;282:431-433. 


\section{CHAPTER 11}

\section{General discussion and conclusions}

When samples of citrated or heparinised whole blood were stirred under controlled conditions a progressive reduction in the platelet count measured using the Ultra-Flo 100 whole blood platelet counter (UF100) was seen (Fox et all 1982). A microscopic examination of the blood samples revealed a strong correlation between the reduction in platelet count and the appearance of visible platelet aggregates. In contrast, when blood was anticoagulated with EDTA and stirred, there was no change in the platelet count, and no aggregates visible on microscopic examination. As the UF100 counts single platelets very accurately in whole blood samples (Day et al 1980) it was concluded that this instrument could be used to measure whole blood platelet aggregation (WBA).

Measuring platelet aggregation using whole blood with a particle counting technique has many advantages when compared with optical aggregometry (Born 1962) which uses platelet-rich plasma (PRP). Whole blood is more like circulating blood than PRP because the entire population of cells from the blood sample are present. The results obtained should therefore be more representative of in vivo platelet behaviour. The use of the WBA technique allowed for more rapid testing and required a smaller blood sample compared with optical aggregometry (Fox et al 1980, Bevan and Heptinstall 1985). The extent of WBA can be easily quantified at time intervals ranging from a few seconds to several minutes, whereas obtaining quantitative data from optical aggregometry traces is not simple.

The extent of the WBA measured was strongly dependent on the methodology used. More rapid WBA was seen when the blood was exposed to plastic rather than glass (Fox et al 1980), and as the blood aged more WBA could be induced by either stirring or by the addition of platelet agonists. The storage conditions and mixing of the blood sample can also influence the extent of WBA (Fox et al 1980 , Butchers et al 1980, Saniabadi et al 1987). The WBA technique was carefully standardised during the initial evaluation and in subsequent investigations, so that conflicting results caused by differences in technique could be recognised or avoided. 
The WBA technique was extremely sensitive to platelet aggregation as it could measure aggregation that was undetectable using optical aggregometry. When blood samples collected from two patients with Glanzmann thrombasthenia were tested the extent of WBA was similar to that seen in normal controls, whilst there was little or no aggregation observed using optical aggregometry (BurgessWilson et al 1987). In other experiments, where diamide was added to samples of whole blood (Losche et al 1985), the WBA technique clearly demonstrated reversible WBA when there was no change in the light transmission of stirred PRP. Microscopic examination of the blood samples used in these experiments confirmed the presence of platelet aggregates. The increased sensitivity of the WBA technique compared to optical aggregometry was probably due to the size of aggregates that can be detected by these techniques. When platelet aggregation is induced in PRP the light transmission only falls when very relatively large platelet aggregates are formed and is not influenced by small platelet aggregate formation. Whereas the reverse is true with the WBA technique, here the number of single platelets falls rapidly as soon as extremely small aggregates form. In contrast to optical aggregometry the WBA technique is relatively insensitive to the formation of large platelet aggregates. This is because the UF100, like most other electronic particle counters, has pre-set particle size thresholds that allow it to count "platelet-sized" particles. If a platelet aggregate exceeds this pre-set size range the instrument no longer counts it. Optical aggregometry and the WBA technique clearly measure different aspects of platelet aggregation and whilst the WBA technique promises to be useful in the investigation of hyper-aggregable platelets because it can measure small increases in platellet aggregation, the optical aggregometry should in theory be more useful in detecting hypoaggregable platelets.

The WBA technique detected platelet aggregation induced by the addition of any platelet agonist that is known to cause platelet aggregation in PRP. Those agonists tested include; adenosine diphosphate (ADP), adrenaline, arachidonic acid, collagen, platelet activating factor, ristocetin, serotonin and thrombin. The extent of the WBA induced was dose-dependent, and the agonists that induce marked aggregation in PRP, notably arachidonic acid and ristocetin, also exhibited the same behaviour in whole blood (Burgess-Wilson et al 1987 , Cockbill et al 1985, Burgess-Wilson et al 1985, Heptinstall et al 1986).

One of the most striking observations in the investigation was 'spontaneous platelet aggregation' (SPA). The term 'spontaneous platelet aggregation' was first used in optical aggregometry studies to describe a change in light transmission, typically a $20 \%$ decrease in optical density that was seen when PRP was stirred 
for between $10-30$ minutes without the addition of any platelet agonist. Stirring citrated whole blood samples caused an immediate, progressive reduction in the platelet count (Fox et al 1982) and the term SPA was used to describe this observation although it was recognised that the aggregation was not 'spontaneous", but was stirring-induced. SPA is another example of the WBA techniques' sensitivity to weak platelet aggregation. When PRP is studied using optical aggregometry SPA is observed only infrequently, but in these investigations SPA was observed in every citrated and heparimised blood sample analysed; both in normal. individuals (Fox et al 1982, Burgess-Wilson et al 1984) and in abnormal individuals (Burgess-Wilson et al 1986, Burgess-Wilson et al 1987, Jones 1986 and Heptinstall et al 1988). Whilst some investigators wished to avoid and remove this phenomena (Butchers et al 1980), we and others attempted to understand and use SPA as a simple measurement of platelet activity (Saniabadi et al 1984, Saniabadi et al 1987, Splawinska et al 1990). The variation in extent of the SPA seen in blood samples from different individuals was considerable; in some blood samples only $10 \%$ of the single platelets were lost after stirring the blood for 10 minutes, whereas in other blood samples $90 \%$ of the single platelets were lost after one minute of stirring. The extent of SPA was relatively constant in blood samples collected from the same individual repeatedly over several weeks (Fox et al 1982). The study of normal individuals revealed a strong correlation between the individuals' age and the extent of SPA in both citrated and heparinised whole blood. A correlation between the extent of SPA and haematocrit $(\mathrm{Hct}$ ) was seen in a preliminary study (Burgess-Wilson et al 1984), but further testing showed that this was an artifact produced by the age/sex distribution of the original group tested (Burgess-Wilson et al, in press). The subject group used in the first study contained a large proportion of young females with low Hct, and older males with higher Hct, and this produced a correlation between age and the Hct. The later study investigated a larger group of normal subjects with a more even age/sex distribution, and no correlation between age and Hct was seen whilst the strong correlation between SPA and age remained. As the incidence of thromboembolic disease increases with age, the measurement of SPA might be able to predict those individuals at risk of developing thrombotic disease.

Adenosine diphosphate (ADP) released from red cells has been implicated by many investigators as one of the main causes of SPA. Stirring of whole blood traumatises the red cells and causes the release of ADP (Gaarder et al 1961, Harrison and Mitchell 1966, Fox et al 1982, Saniabadi et al 1985). This hypothesis has been supported by experiments showing that ADP-removing enzyme systems added to stirred whole blood partially inhibit SPA (Fox et al 1982, Saniabadi et al 1984, Jen and McIntire 1984). Experiments using 
dipyridamole may also help to explain the causative mechanism of SPA. Dipyridamole has been extensively studied as an inhibitor of platelet aggregation and has been shown to reduce the mortality rate in thromboembolic disease (Antiplatelet Trialists" Collaboration 1988). However, dipyridamole does not usually inhibit platelet aggregation in PRP (Saniabadi et al 1987, Weiss 1982, Harker and Kadatz 1983) because its affect on the platelet is indirect. Its target appears to be the red blood cell and it has been suggested that dipyridamole inhibits adenosine uptake of blood cells which leads to a build up in the plasma of adenosine which is an anti-platelet agent. This hypothesis was supported by experiments which showed that the addition of adenosine potentiated the inhibitory effect of dipyridamole (Heptinstall et al 1986). Saniabadi et al (1987) have shown that dipyridamole can inhibit SPA in a time dependent manner, but their results suggest that adenosine is not involved in the anti-SPA effect of dipyridamole, instead they suggest that dipyridamole directly inhibits the ADP leakage from red blood cells that causes SPA. Violi et al (1991), using the impedance aggregometry technique, found that dipyridamole had less effect on WBA than Saniabadi had found. Violli suggested that dipyridamole reduces red cell membrane permeability, thus reducing the ADP leakage. Oxygen-free radicals can cause peroxidation of membrane phospholipids that results in an increased membrane fluidity and permeability, and loss of membrane integrity, all of which favour ADP leakage (Kalra et al 1994). Dipyridamole has anti-oxidant properties and reduces the levels of these oxygen-free radicals. Experiments using the WBA technique suggested that dipyridamole inhibits WBA, either as SPA or agonist-induced aggregation. The concentrations of dipyridamole used where much higher than those achievable in vivo, which suggests that this observation may be an in vitro artifact. There are other possible mechanisms by which SPA in whole blood might be influenced. Phospholipid-derived material from the red blood cell membrane, such as arachidonic acid, has been shown to activate platelets due to thromboxane $A_{2}$ synthesis in platelets (Alkhamis et al 1990). The binding of prostacyclin by red cells can produce a pro-aggregatory environment; and the effect of the shear forces induced by the presence of red cells can be proaggregatory. At present, however, the relationship between these mechanisms, ADP leakage and the extent of SPA is unclear.

The WBA technique was used to investigate the effect of various substances on platelet behaviour and changes in platelet behaviour due to different disease states. In one study a preparation containing eicosapentaenoic was administered to several healthy normal volunteers for two weeks, despite marked changes in the platelet fatty acid composition, no change in the extent of SPA or agonistinduced platelet aggregation was seen (Cockbill et al 1985, Maurice et al 1987). The beneficial effects of fish oil ingestion on the incidence of heart disease either 
relates to the modification of other aspects of platelet behaviour or is unrelated to changes in platelet behaviour. It is possible that it is the changes in cholesterol, triglycerides or blood viscosity may be causing the observed reduction in the incidence of thrombosis.

In another study subjects were studied sequentially after myocardial infarction (MI). In the first 24 hours after the event there was no difference in platelet activity seen in patients who had had an MI and the normal control group. However, the extent of WBA measured in citrated blood obtained within 24 hours of the MI was higher in patients who subsequently died in the four weeks after the MI compared with those who did not die. Unfortunately, although the difference was significant, it was not big enough to clearly identify those individuals who had a poor prognosis (Jones 1986). Administration of the thromboxane synthetase inhibitor Dazoxiben to these patients inhibited both the $\mathrm{TxB}_{2}$ synthesis in whole blood and the release of agonist-induced $5 \mathrm{HT}$ from PRP, but had no effect on either WBA or the incidence of subsequent venous thrombosis. The lack of effect of Dazoxiben on WBA suggested that the extent of WBA is not dependent on thromboxane synthesis.

By chance, it was possible to study an indiviclual who suffered from essential thrombocythaemia both before and after a stroke (Heptinstall et al 1988). The patients' blood was shown to be hyper-aggregable before the stroke, and hypoaggregable afterwards. It is possible that the hyper-aggregable platelets tested before the stroke contributed to and where consumed in the thrombus formation and were not available to be tested afterwards. If this is confirmed it might be possible to use the test to screen patients at high risk of developing thrombosis. Once identified, individual at risk could be treated with prophylactic anticoagulation and anti-platelet therapy.

Heparin-induced platelet aggregation was studied using the WBA technique. It was observed that during pregnancy the addition of heparin to whole blood could induce marked platelet aggregation. The extent of SPA measured using the WBA technique was usually similar in citrated and heparinised blood samples. Blood samples collected from pregnant women stirred in the presence of heparin showed increased SPA compared to normal. The extent of SPA increased during pregnancy, and returned to the level seen in the age-matched controls six weeks post-natal. The same effect was seen in both normotensive and hypertensive subjects, except that the increase in SPA developed slightly later in the course of the pregnancy of the hypertensive group (Burgess-Wilson et al 1986). Further investigation suggested that the in vitro heparin-induced platelet aggregation (HIA) was related to the level of some, as yet, undefined plasma component. The 
extent and speed that the HIA developed varied between different individuals, and it could be inhibited by prostacyclin, apyrase and dipyridamole, but was not inhibited by aspirin. HIA could be quantified by measuring a 'HIA threshold' which was defined as the smallest amount of heparin needed to cause a $10 \%$ loss of single platelets. The highest thresholds were seen in male subjects. Low HIA thresholds, indicating that the platelets are more sensitive to heparin, were seen in pregnant females, which supported the original report of HIA in pregnancy. Low HIA thresholds were also seen in some non-pregnant females and this observation perhaps could be used to identify those individuals who might be at risk of developing heparin-induced thrombocytopenia (HIT). HIT being one of the most important drug reactions occurring today and can be a serious complication of heparin therapy. Although many investigators are developing tests that can detect the presence of HIT, there is at present no single, universally accepted test of choice (Kelton 1994). The measurement the HIA threshold may not only help to identify individuals at risk of developing HIT, but may be of use in the assessment of the effects of anti-platelet treatment in HIT.

No new platelet disorder was discovered using the WBA technique. There are several possible explanations for this. The most common inherited bleeding disorder that can be detected using optical aggregometry is von Willebrand Disease, and the incidence of patients in which laboratory defects can be identified is only 1 in 125 (Sadler 1994). The most common inherited platelet defects, Glanzmann thrombasthenia and Bernard-Soulier syndrome are so rare that often no incidence is cited for the normal population. In the consanguineous population of Iraqi-Jews now living in Israel where the incidence is considered high, the thomozygote frequency is only 1 in 7714 (Coller 1994). A total of about 300 individuals were tested during these investigations, however, the diseases associated with abnormal platelet aggregation are very rare, so this number might be too low to expect the detection of a platelet disorder. During these studies it was usual to measure only SPA, so relatively few individuals were tested with platelet agonists. The platelet abnormalities detected using optical aggregometry are normally associated with defective agonist-induced aggregation. Another explanation is that the WBA technique may be so sensitive to platelet aggregation that it cannot detect the disorders where there is a moderate or even marked hypo-aggregability. This hypothesis is supported by the fact that almost normal WBA was seen in patients with Glanzmann thrombasthenia. It could be speculated that if aggregation was reduced to the extent that even the microaggregates detectable by the WBA technique where unable to form the condition may be so severe as to be incompatible with life. 
The WBA technique described and used in these studies (Fox et al 1982, Bevan and Heptinstall 1985) can be used with any instrument that can accurately count platelets. Other investigators have shown that using similar techniques, results can be obtained on the larger haematology analysers such as the ELT $8 / d$ s and the Coulter T-890 (Barradas et al 1992, Cheeseman et al 1984). These methods have proved, and are proving useful in the research laboratory environment, but they cannot be used easily for large scale testing. Although WBA can be measured using the larger haematology analysers the normal laboratory workload does not allow the large amount of extra time necessary to use these machines for this purpose, and these instruments are too expensive to be used for dedicated platelet studies only. With the advent of the cheaper, more compact multi-parameter haematological analysers few laboratories now have machines like the Ultra-Flo 100 that could be wholly dedicated to platelet aggregation studies. A fully automated system that could measure WBA is quite feasible using present technology and if suitable instrumentation became available the WBA technique might enjoy greater use.

Whilst WBA techniques bring the in vitro testing closer to the in vivo situation there are still several important in vitro factors that will influence the platelet behaviour observed in the test system. Blood is always traumatised by the collection technique as it is forced through the needle. The properties of the blood are changed by the addition of any anticoagulant. The cells in a blood sample have been removed from the influences of their normal environment, i.e. the shear stress found in the circulation and the contact with the vessel wall. These influences are difficult to remove unless one uses one of the highly individual and sophisticated flow systems (Baumgartmer 1973, Hornstra 1975, Kratzer 1985, Görög 1986, Cadroy and Hanson 1990) or a viscometer (Slack et al 1993, Russell-Smith et al 1981, Roth 1991, Jen and McIntire 1984, Weiss et al 1989). The use of both flow systems and viscometers generally requires a high level of expertise which invariably leads to limited use of these techniques in the routine investigation of platelets.

The studies described have shown that meatsuring WBA can reveal aspects of platelet behaviour that cannot be investigated using optical aggregometry. The investigation of platelet aggregation which is not detectable using optical aggregometry might help elucidate the in vivo relevance of the in vitro aggregation process. The clinical utility of this technique has not yet been proved. One approach would be to assess its potential as a thrombotic risk indicator by studying WBA induced by platelet agonists in normal individuals and those individuals with known thrombotic risk, and by the further investigation of the relationship between SPA to age. The further investigation of heparin-induced 
WBA could help provide an explanation of the plasma changes associated with heparin-induced thrombocytopenia. The development of the heparin threshold measurement as a simple routine test would allow an assessment of its potential in the identification of patients at risk of life threatening heparin-induce thrombocytopenia. It can be concluded that the study of whole blood platelet aggregation is still an area of research that holds many challenges and is probably capable of revealing new aspects of platelet behaviour.

\section{References}

Alkhamis TM, Beissinger RL and Chediak JR. Artificial surface effect of red blood cells on platelets in laminar shear flow. Blood 1990; 75: 1568-1575.

Antiplatelet Trialists' Collaboration. Secondary prevention of vascular disease by prolonged antiplatelet treatment. BMJ 1988; 296: 320-331.

Barradas MA, O'Donoghue S, Jagroop A and Mikhailidis DP. Laboratory techniques in drug development (2): Advantages of whole blood platelet aggregation measured by a cell counter (Coulter T-890) in drug evaluation. J Drug Development 1992; 5: 155-166.

Baumgartner HR. The role of blood flow in platelet adhesion, fibrin deposition and formation of mural thrombi. Microvasc Res 1973; 5: 167-179.

Bevan $\mathbf{J}$ and Heptinstall $\mathrm{S}$. Serotonin-induced platelet aggregation in whole blood and the effects of ketanserin and mepyramine. Thromb Res $1985 ; 38 ; 189-194$.

Born GVR. Aggregation of blood platelets by adenosine diphosphate and its reversal. Nature (Lond) 1962; 194: 927-929.

Burgess-Wilson ME, Green S, Heptinstall $S$ and Mitchell JRA. Spontaneous platelet aggregation in whole blood: Dependence on age and haematocrit. Lancet Nov 24, 1984; ii: pp 1213.

Burgess-Wilson ME, Morrison $\mathrm{R}$ and Heptinstall $\mathrm{S}$. Spontaneous platelet aggregation in heparinised blood during pregnancy. Thromb Res 1986a; 37: 385-393.

Burgess-Wilson ME, Green S, Heptinstall $S$ and Mitchell JRA. Spontaneous platelet aggregation in whole blood obtained from 102 normal individuals : A strong correlation with Age. Submitted to Haemostasis 1995.

Burgess-Wilson ME, Cockbill SR, Johnston GI and Heptinstall S. Platelet aggregation in whole blood from patients with Glanzmann's thrombasthenia. Blood 1987; 69: 38-42. 
Butchers J, Humphrey PPA, Hyde J, Lumley $\mathrm{P}$ and Spurting NW. The evaluation of a new electronic counting technique for measurement of platelet aggregation in human whole blood in vitro. Br J Pharmacol 1980; 70: 160-161.

Cadroy $Y$ and Hanson SR. Effects of red blood cell concentration on hemostasis and thrombus formation in a primate model. Blood 1990; 75(11): 2185-2193.

Coller BS. Inherited disorders of platelet function. In: Haemostasis and thrombosis. Bloon AL, Forbes CD, Thomas DP and Tuddenham EGD, eds. Churchill Livingstone, New York, 1994; pp $721-766$.

Cheeseman JE, Mills SP and Hardisty RM. Platelet aggregometry on whole blood: the use of the ELT $8 / \mathrm{ds}$ blood cell counter in the investigation of bleeding disorders. Clin Lab Haematol 1984; 6: 265-272.

Cockbill SR, Heptinstall S and Allen BR. The effects of Dietary fish oil supplementation on human blood platelet behaviour in healthy volunteers and in patients with psoriasis. (Abstract). BSHT Annual General Meeting, London, 25-26 September 1985.

Day HI, Young E and Helfrich M. An evaluation of a whole-blood platelet counter. Am J Clin Pathol 1980; 73: 588-593.

Fox SC, Burgess-Wilson ME, Heptinstall S and Mitchell JRA. Platelet aggregation in whole blood determined using the Ultra-Flo 100 platelet counter. Thromb Haenost $1982 ; 48$; 327 329.

Gaarder A, Jonsen J, Laland S, Hellem A and Owren PA. Adenosine diphosphate in red cells as a factor in the adlhesiveness of human blood platelets. Nature $1961 ; 1,92: 531-532$.

Görög P. A new, ideal technique to monitor thrombolytic therapy. Angiology 1986; 37: 99. 105.

Harker LA and Kadatz RA. Mechanism of the action of dipyridanole. Thromb Res 1983; Suppl IV: pp 3946.

Harrison MJG and Mitchell JRA. The influence of red blood-cells on platelet adhesiveness. Lancet, Nov 26, 1966; pp. 1163-॥164.

Heptinstall S, Fox S, Crawford J and Hawkins M. Inhibition of platelet aggregation in whole blood by dipyridamole and aspirin. Thromb Res $1986 ; 42: 215-223$.

Heptinstall S, Cockbill SR, Burgess-Wilson ME and Jones EW. Studies on platelets betore and after stroke in a patient, with essential thrombocylhaemia. BMJ 1988; 296: 1298-1299.

Hornstra $G$ and ten Hoor $F$. The Filtragometer. A new device for measuring platelet aggregation in venous blood of man. Thrombos Diathes Haemorrh (Stuttg) 1975; 34: 531 544. 
Jen CJ and Mclntire LV. Characteristics of shear-induced aggregation in whole blood. J Lab Clin Med 1984; 103: 115-124.

Jones EW. Venous thromboembolism: Studies of aspects of its pathogenesis and treatment. Thesis submitted to the University of Notingham for the degree of Doctor of Medicine, August 1986.

Kalra J, Mantha SV and Prasad K. Oxygen-free radicals: Key factors in clinical diseases. LabMedica International March/April 1994; pp. 16-21.

Kelton JG. Heparin induced thrombocytopenia. Hemostasis and thrombosis update 94. 11 th Annual 3 Day Seminary and Workshop. April 1994, pp 15-19.

Kratzer MAA and Born GVR. Simulation of primary haemostasis in vitro. Haemostasis 1985 ; 15: 357-362.

Lösche W, Burgess-Wilson ME, Michel E, Heptinstall S and Till U. Diamide induces reversible aggregation of human blood platelets. Thromb Res 1985; 40: 869-874.

Maurice PDL, Allen BR, Barkley ASJ, Cockbill SR, Stammers J and Bather PC. The effects of dietary stupplementation with fish oil in patients with psoriasis. $\mathrm{Br}$ J Dermatol $1987 ; 117 ; 599$ 606.

Poller L, Thomson JM and Priest CM. Coumarin therapy and platelet aggregation. BMJ, 22 Feb 1969, pp 474-476.

Newland $\mathrm{H}$ and Nordöy A. Effect of large doses of warfarin sodium on haemostasis and on ADP-induced platelet aggregation in vivo in the rat. Cardiovasc Res 1967; 1: 362-370.

Roth GJ. Developing relationships : Arterial platelet adhesion, glycoprotein Ib, and lencine-rich glycoproteins. Blood $1991 ; 77(1) ; 5-19$.

Russell-Smith NC, Flower RJ and Cardinal DC. Measuring platelet and leucocyte aggregation/adhesion responses in very small volumes of whole blood. J Pharmacol Methods $1981 ; 6: 315-333$.

Sadler JE. von Willebrand disease. In: Haemostasis and thrombosis. Bloom AL, Forbes CD, Thomas DP and Tuddenham EGD, eds. Churchill Livingstone, New York, 1994; pp 843-857.

Saniabadi AR, Lowe GDO, Barbenel JC and Forbes CD. A comparison of spontaneous platelet aggregation in whole blood with platelet rich plasma: additional evidence for the role of ADP. Thromb Haemost 1984; $51: 115-118$.

Saniabadi AR, Lowe GDO, Barbenel JC and Forbes CD. Further studies on the role of red blood cells in spontaneous platellet aggregation. Thromb Res $1985 ; 38: 225-232$. 
Saniabadi AR, Lowe GDO, Barbenel JC and Forbes CD. Effect of dipyridamole on spontaneous platelet aggregation in whole blood decreases with the time after venepuncture: evidence for the role of ADP. Thromb Haemost 1987; 58: 744-748.

Slack SM, Cui Y and Turitto VT. The effects of flow on blood coagulation and thrombosis. Thromb Haemost 1993; 70(1): 129-134.

Splawinska B, Kuzniar $J$ and Splawinska D. Is spontaneous platelet aggregation present in whole blood in vitro. Thromb Haemost $1990 ; 64(1): 180$.

Violi F, Pratico D, Iuliano L and Balsano F. Dipyridamole potentiates the inhibition of platelet aggregation by aspirin (in human platelet rich plasma and whole blood). J Lipid Mediat $199 \|$; 4: $61-68$.

Weiss HJ. Dipyridamole and other phosphodiesterase inhibitors. In: Platelets Pathophysiology and antiplatelet drug therapy. Harvey J Weiss (Ed), Alan R Liss Inc, New York, 1982; pp 5762.

Weiss HJ, Hawiger J, Ruggeri ZM, Turitto VT, Thiagarajan P and Hoffmann T. Fibrinogenindependent platelet adhesion and thrombus formation on subendothelium mediated by glycoprotein Irb-lIla complex at high shear rate, J Clin Invest 1989; 83: 288-297. 
Whole blood platelet aggregation (WBA) was evaluated using a platelet counting technique. The instrument used, the Ultra-Flo 100 , counts single platelets in whole blood samples. Microscopic examination of the blood samples confirmed that the loss of single platelets in stirred whole blood was proportional to the degree of platelet aggregation. This method of meastiring WBA had several advantages over optical aggregometry using platelet-rich plasma (PRP). The method allowed more rapid testing and used a smaller blood sample. The whole blood is closer to the natural milieu of circulating blood than PRP, having all the different cells present in the correct relative proportions. The method also yielded a quantifiable result, unlike optical aggregometry and impedance aggregometry where a subjective analysis of the aggregation 'trace' is often necessary. It was necessary to carefully define the experimental conditions used because different methods of measuring platelet aggregation could provide quite different results.

The WBA method detected platelet aggregation in response to agonists known to aggregation platelets (in PRP) using optical systems. However, the method proved to be a very sensitive detector of weak platelet aggregation. It could measure platelet aggregation that was undetectable to optical systems; in blood collected from patients with Glanzmann Thrombasthenia and in blood when platelet aggregation was stimulated by the addition of diamide. The WBA method detected the formation of very small platelet aggregates, in contrast to optical aggregometry which only detected the formation of large platelet aggregates. One of the most striking observations was the so called, 'spontaneous' platelet aggregation (SPA), where platelet aggregation is observed in response to stirring or the standing of blood. SPA occurred in every citrated and heparinised blood sample tested, but not in blood anticoagulated with EDTA; whereas when optical aggregometry was used it was uncommon to detect any SPA. In a study of normal individuals it was found that the extent of SPA increased with the subjects age, but was independent of several thrombotic risk markers including haematocrit and fibrinogen. It was proposed that SPA represents the sensitivity of the platelets to aggregate and that this measurement may be important in the detection of individuals with hyper-aggregable platelets who are at risk of developing thrombotic disease. The main cause of the SPA in stirred blood appears to be 
adenosine diphosphate released from the red blood cells, and several experiments provided evidence that inhibitors of platelet function, notably aspirin, could reduce the extent of SPA.

WBA was investigated in several clinical conditions with pregnancy providing a most interesting observation. It was demonstrated that heparin could induce marked platelet aggregation in whole blood collected from pregnant subjects. It was further shown that a heat-stable, as yet undefined, plasma component could influence the extent of heparin-induced aggregation (HIA), and the WBA method could used to quantify the extent of HIA. It was proposed that this method might have an application in the detection of some individuals likely to experience heparin-induced thrombocytopenia, a common and sometimes serious complication of heparin therapy.

It was concluded that the WBA method has its main use in detecting hyperaggregable platelets, and that optical aggregometry was more suitable for the detection of hypo-aggregable platelet disorders. The WBA method has an important role in the research of thrombotic disease. However, although the method can be adapted to any particle counter that can detect platelet-sized particles, it is not used as extensively as Born (optical) aggregometry. The availability of instrumentation to measure optical platelet aggregation is probably the main reason. A fully automated version of the Ultra-Flo 100 would encourage greater use of this technique and the studies presented in this thesis indicate that there are many areas of platelet research where this whole blood aggregation method could be useful. 
Dit proefschrift beschrijft de evaluatie van een techniek (plaatjesaggregatie in volbloed, PAV) om bloedplaatjesaggregatie te meten in niet gecentrifugeerd bloed, door middel van het meten van de afname van vrije, individuele plaatjes. De plaatjes werden geteld met behulp van een instrument, de Ultra Flow 100, dat werkt volgen het "Coulter Counter" principe. De afname van het aantal vrije plaatjes in volbloed correleerde goed met het verschijnen van plaatjesaggregaten in bloeduitstrijkjes. Deze methode heeft verscheidene voordelen boven de meer gebruikelijke optische aggregometrie in plaatjesrijk plasma (PRP): zij is sneller en er is een kleiner bloedmonster nodig. Bovendien benaderd volbloed het natuurlijk milieu beter dan PRP.

PAV geeft een kwantitatief resultaat, in tegenstelling tot de optische aggregometrie en het andere alternatief, de impedantiemeting in volbloed, die beiden semikwantitatief zijn en soms een subjectieve analyse van de verkregen curven vereisen.

Kwalitatief geeft PAV dezelfde resultaten als de optische methode; dezelfde agonisten induceren aggregatie etc. Ook na zorgvuldige optimalisatie en standaardisatie van de proefomstandigheden bleken de resultaten van PAV echter kwantitatief te verschillen van die verkregen met de optische methode. PAV blijkt een zeer gevoelige detector van een geringe plaatjesaggregatie. De zwakke aggregatie die voorkomt in het bloed van patiënten met Glanzmanns'thrombasthenie of die in normaal plasma kan worden geïnduceerd door diamide ontsnapt aan de optische aggregometrie omdat deze slechts grote plaatjesaggregaten aantoont terwijl PAV ook de vorming van kleine aggregaten meet.

Een van de meest opvallende waamemingen was de "spontane plaatjesaggregatie" (SPA) die zich ontwikkeld in bloed dat, al of niet geroerd, wordt bewaard. Dit verschijnsel ontsnapt meestal aan de waarneming met de optische methode. SPA wordt gevonden in citraat- zowel als in heparineplasma maar niet in EDTA plasma. De belangrijkste oorzaak van het verschijnsel is het vrijkomen van adenosine difasfaat uit de rode bloedcellen. De studie van dit verschijnsel in een normale populatie liet zien dat SPA toeneemt met de leeftijd maar niet correleert met indicatoren van thrombose risico zoals hematokriet en fibrinogeengehalte. De resultaten suggereren dat SPA een indicator is voor de aggregatieneiging van plaatjes en dat de meting ervan van belang kan zijn voor het opsporen van individuen die door een verhoogde aggregeerbaarheid van de bloedplaatjes een verhoogd risico lopen op thromboembolische aandoeningen. Remmers van de plaatjesfunctie zoals aspirine kunnen de mate van SPA verminderen. 
Wij bepaalden de PAV in een aantal verschillende klinische condities. De waarnemingen bij zwangeren verdienen aparte vermelding. Wij konden laten zien da: heparine plaatjesaggregatie kan induceren in het bloed van zwangere vrouwen en dat dif verschijnsel afhankelijk is van een, vooralsnog niet nader gedefinieerde, hittestabiele plasmacomponent. Waarschijnlijk kan PAV gebruikt worden om patiënten op te sporen die een neiging hebben om een heparine-geïnduceerde thrombocytopenie te ontwikkelen.

Samenvattend kan gezegd worden dat PAV bij uitstek geschikt is voor de detectie van hyperaggregeerbare plaatjes terwijl optische aggregometrie afwijkingen detecteert die gepaard gaan met verminderde plaatjesaggregatie, die overigens ook aan PAV niet ontsnappen. PAV kan potentieel een belangrijke rol spelen bij het onderzoek naar thromboembolische ziekten. De techniek kan worden uitgevoerd in ieder laboratorium dat beschikt over een blloedcelteller die in staat is om deeltjes te onderscheiden met de grootte van individuele bloedplaatjes. Toch is de techniek veel minder ingeburgerd dan de optische aggregometrie volgens Born. De reden zow kunnen zijn dat voor deze laatste techniek specifieke apparatuur verkrijgbaar is. Het verdient daarom overweging zulke apparatuur ook voor de PAV techniek te ontwikkelen. 


\title{
Acknowledgments
}

The research described in this thesis was performed in the Department of Medicine, Queens Medical Centre, University Hospital, Nottingham, England, in close collaboration with John Adams, Steve Cockbill, Sue Fox, Jackie Glenn, Maxine Hawkins, Stan Heptinstall, Martin Lawrence, Jane May, Marianne McPherson, Rachel Morrison, John Westby and Emyr Wynn-Jones.

This thesis required a good deal of time, effort and commitment. In my family there are two young children, so the effort on my part required an equal effort from the main support of my life :

\section{Pamela}

The positive effect of many other people in my life has helped me to be able to produce this thesis but I feel that the following deserve a special acknowledgment

\section{My father and mother - Ashton and Helen Anita Wilson}

\author{
and my colleagues \\ John Adams \\ Sue Fox \\ Stan Heptinstall \\ Jane May \\ J.R.A. Mitchell \\ Pete Nichols \\ Piet van de Waart \\ John Westby \\ Barry Woodhams
}

And I would especially like to thank my promoters for their help and encouragement during the preparation of this thesis

Prof. Dr. H.C. Hemker and Prof. Dr. S. Heptinstall 


\section{Curriculum Vitae}

The author was born on April 29th, 1956 in England. In 1974 he left school to work as a Junior Laboratory Technician in the Haematology Department of the General Hospital, Nottingham, England. He became a fellow of the Institute of Biomedical Sciences in 1978, and continued his career investigating platelet behaviour in the Department of Medicine, University Hospital, Nottingham. The work presented in this thesis was carried out whilst he worked in that department. In 1986 he was appointed to the position of Senior Technician in the Haemostasis Laboratory of the Haematology Department, University Hospital, Nottingham. In 1990 he was appointed to the position of Research Scientist in the Haemostasis Research and Development Department of Dade International Inc., Düdingen, Switzerland. In November 1995 he was appointed the position of Senior Scientist in an Applied Research and Development Department of LifeScan Inc, California, USA a Johnson \& Johnson company. Under the encouragement of Prof. H.C. Hemker, Prof. S. Heptinstall and Dr. B. Woodhams he was able to collate his previous work and present it in this thesis. 